\title{
WestVirginiaUniversity
}

THE RESEARCH REPOSITORY @ WVU

Graduate Theses, Dissertations, and Problem Reports

2021

\section{Effects of Nonexamples on Concept Formation}

Catherine Louise Williams

West Virginia University, cs0098@mix.wvu.edu

Follow this and additional works at: https://researchrepository.wvu.edu/etd

Part of the Applied Behavior Analysis Commons, Curriculum and Instruction Commons, and the Experimental Analysis of Behavior Commons

\section{Recommended Citation}

Williams, Catherine Louise, "Effects of Nonexamples on Concept Formation" (2021). Graduate Theses, Dissertations, and Problem Reports. 8337.

https://researchrepository.wvu.edu/etd/8337

This Dissertation is protected by copyright and/or related rights. It has been brought to you by the The Research Repository @ WVU with permission from the rights-holder(s). You are free to use this Dissertation in any way that is permitted by the copyright and related rights legislation that applies to your use. For other uses you must obtain permission from the rights-holder(s) directly, unless additional rights are indicated by a Creative Commons license in the record and/ or on the work itself. This Dissertation has been accepted for inclusion in WVU Graduate Theses, Dissertations, and Problem Reports collection by an authorized administrator of The Research Repository @ WVU.

For more information, please contact researchrepository@mail.wvu.edu. 
Graduate Theses, Dissertations, and Problem Reports

2021

Effects of Nonexamples on Concept Formation

Catherine Louise Williams

Follow this and additional works at: https://researchrepository.wvu.edu/etd

Part of the Applied Behavior Analysis Commons, Curriculum and Instruction Commons, and the Experimental Analysis of Behavior Commons 
Effects of Nonexamples on Concept Formation

\author{
Catherine Williams
}

Dissertation submitted to the Eberly College of Arts and Sciences at West Virginia University in partial fulfillment of the requirements for the degree of

Ph.D. in Psychology- Behavior Analysis

\author{
Michael Perone, Ph.D., Co-Chair \\ Claire St. Peter, Ph.D., Co-Chair \\ William Beasley, Ed.D. \\ Kathryn Kestner, Ph.D. \\ Shari Steinman, Ph.D.
}

Department of Psychology

\title{
Morgantown, West Virginia 2021
}

Keywords: Concept formation, conceptual learning, examples, nonexamples, human operant, generalization, instructional design

Copyright: 2021 Catherine Williams 


\title{
ABSTRACT \\ Effects of Nonexamples on Concept Formation
}

\begin{abstract}
Catherine Williams
Concept formation is affected by the examples and nonexamples provided during training, but the degree to which examples and nonexamples should differ is unknown. Two experiments compared concept formation when different kinds of nonexamples were used during training. Both experiments included a within-subject comparison of concept formation following three training conditions: a) nonexamples that were more similar to the examples, b) nonexamples that are less similar to the examples, and c) no nonexamples. Arbitrary concepts were trained in Experiment 1 and concepts akin to those that could be taught in a classroom were trained in Experiment 2. Before and after training, tests with untrained examples and nonexamples measured concept formation. In general, concept formation improved when the concept was taught using nonexamples compared to training with only examples, and nonexamples that were more similar to the examples resulted in the highest levels of concept formation. However, for some participants in both experiments, concept formation was similar across conditions, and concept formation may have been influenced by condition sequence. These findings indicated that the relation between stimuli used to teach concepts affects concept formation. It is recommended that both instructors and researchers consider these relations when selecting stimuli to promote concept formation in instructional and experimental arrangements.
\end{abstract}




\section{Acknowledgements}

I owe the completion of this dissertation to the support I have received from my advisors, colleagues, and family.

I would first like to thank my co-chairs, Drs. Claire St. Peter and Michael Perone, for their undying support throughout the many phases of development and modification of the procedures that I ultimately used for this project. In addition, their patience and commitment to quality feedback throughout the writing process has undoubtedly made me a better writer.

I would also like to thank the undergraduate lab assistants who helped me test the experimental procure and conduct experimental sessions. Without them, I would not have been able to complete this project in the time that was available and maintain my sanity.

Last, but certainly not least, I would like to thank my husband, Xavier Williams, for providing constant support, especially when I encountered unexpected challenges. He created a supportive environment for me "to draw crazy triangles" in and made himself available to be a sounding board during this adventure. 


\section{Table of Contents}

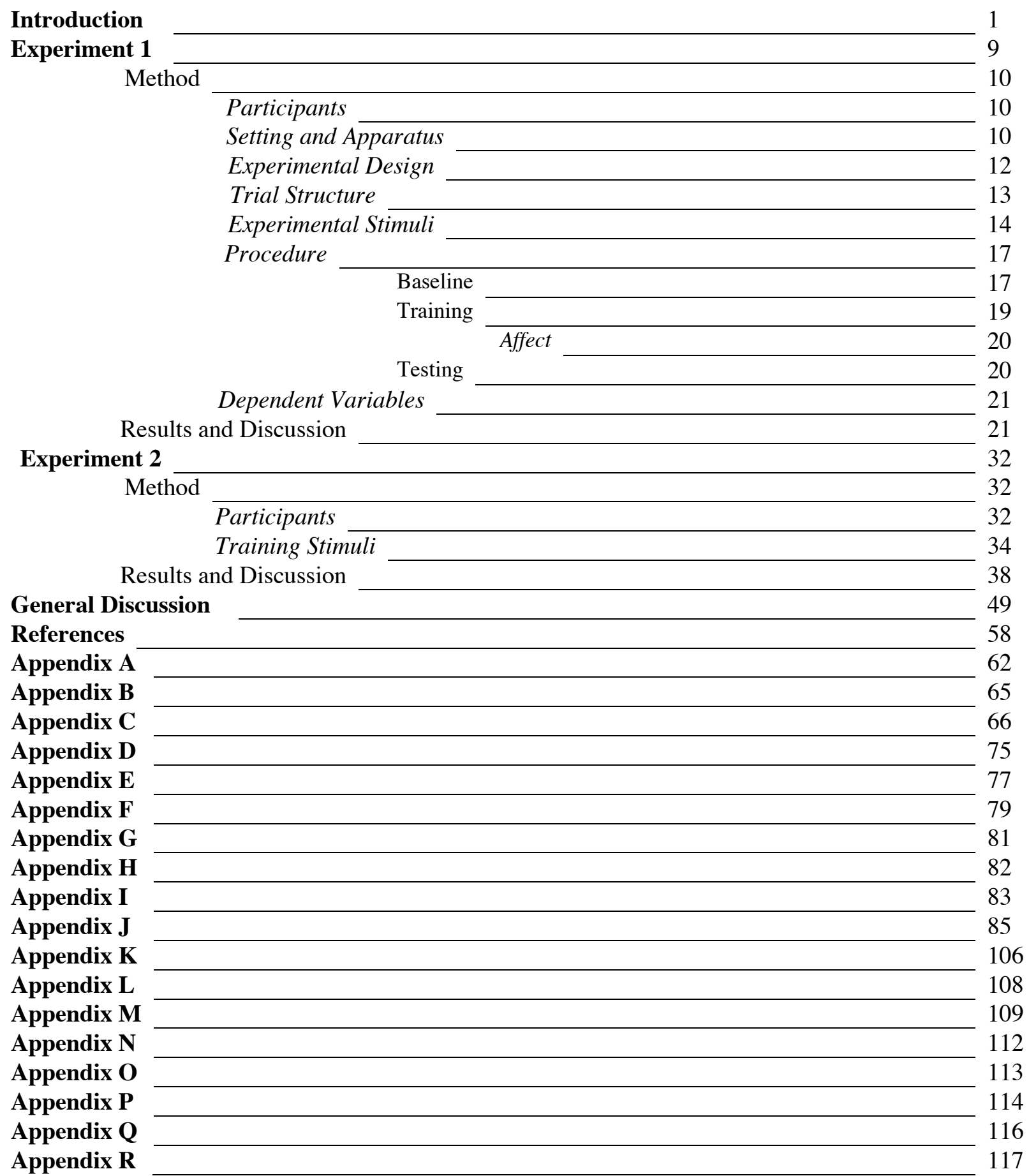




\section{Effects of Nonexamples on Concept Formation}

Effective instruction is critical to ensure that students learn concepts. Learning a concept is more than simply repeating a definition (Markle \& Tiemann, 1970b). Students have learned a concept only if they both generate and respond correctly to new stimuli related to the concept. Stimuli within a conceptual class are alike in particular ways, such as having a shared function (e.g., "tool”), relation (e.g., "same”), or perceptual features (e.g., "square”; Fleming \& Levie, 1993). Concepts defined in terms of shared perceptual features can be defined in terms of shared (relevant) and unshared (irrelevant) features. Once these relevant and irrelevant features have been identified, they can be used to select stimuli to teach the concept.

By definition, concept formation has occurred when a particular response happens in the presence of untrained examples but not in the presence of untrained nonexamples (Fleming \& Levie, 1993; Markle \& Tiemann, 1970a; Mechner, 1965; Woolley \& Tennyson, 1972; Zentall et al., 2002). For instance, when teaching the concept of "square," students can demonstrate concept formation by calling a novel picture of a square a square, but not a novel picture of a triangle. Concept formation does not necessarily require that the responder communicate the definition of the concept or describe why a stimulus is or is not an example. Because concept formation does not require language, methods to teach concepts to all populations can also be informed by research with nonhumans.

Laboratory studies of concept formation using both nonhumans (most frequently pigeons) and humans typically consist of training and test phases (see Khallad, 2004; Wasserman, 2016; Zentall et al., 2002 for reviews) akin to instruction and assessment in a classroom. In general, training consists of trials that begin with the presentation of at least one stimulus and responding (consisting of pecking, clicking, or touching) to the stimulus is measured until the end of the 
trial. The trial may end after a single response or after a fixed amount of time (allowing for multiple responses to be made). The number of stimuli shown at a time and definition of a correct response varies across procedures. Training typically continues until correct responses occur in a high percentage of trials. After training, test trials with untrained stimuli are presented. In most cases, test trials are conducted using procedures that parallel those used on training trials. Some untrained stimuli are examples and others are nonexamples of the concept. So that individuals do not learn which untrained stimuli are examples and nonexamples, either all or none of the responses to the untrained stimuli are followed by reinforcement (regardless of whether the response is correct). If responding continues in the presence of untrained examples, this is evidence of generalization within a concept. If responding also does not occur in the presence of novel nonexamples, this is evidence of discrimination between untrained examples and nonexamples. When there is evidence of both generalization and discrimination, concept formation has occurred.

Concept formation is affected by the stimuli presented during training. It has been theorized that using more stimuli during training increases the likelihood of concept formation (e.g., Stokes \& Baer, 1977). Previous research found concept formation was more likely to occur after training was completed with multiple distinct groups of stimuli (i.e., Multiple Exemplar Training; e.g., Cedro et al., 2019). In addition, the number of training stimuli included simultaneously during training was manipulated in several experiments with rats and pigeons (e.g., Bodily et al., 2008; Lazarowski et al., 2019; Nakamura et al., 2009). Bodily et al. and Nakamura et al. gradually raised the number of stimuli, or "set size", used during training with pigeons. Testing occurred after training with each set size. In both experiments, as training set size was raised, test accuracy increased. Similarly, Lazarowski et al. trained rats to select which 
of two comparison olfactory stimuli matched the sample stimulus. They found that rats performed better on test trials with untrained stimuli when trained with 10 sample stimuli than when trained with two sample stimuli.

As the number of stimuli increases, the number of examples and nonexamples used in training increases proportionally. However, it is not clear if increasing only the number of examples or the number of nonexamples would have the same effect. Effects of multiple examples (without nonexamples) on concept formation have been studied in the context of classroom instruction by Shumway and Lester (1974). College students were taught two mathematical concepts (commutativity and associativity). During training, the students were shown 20 equations and identified each equation as an example of commutativity or associativity. Following each response, they were told whether their answer was correct. They were taught each concept using examples only or both examples and nonexamples. In the examples-only condition, all 20 equations were examples; in the examples and nonexamples condition, 10 equations were examples and 10 were nonexamples. After training, students were tested with 10 untrained examples and 10 untrained nonexamples. Using only examples during training hindered concept formation. Compared to students trained with examples only, the students trained with both examples and nonexamples scored better on the test with untrained equations even though fewer examples were used in their training.

Examples and nonexamples each serve a different function in concept formation. When many examples are used during training, individuals are likely to identify the features that are constant across these examples as the relevant features of the concept (Markle \& Tiemann, 1970a; Markle \& Tiemann, 1970b; Tennyson et al., 1972; Song et al., 2020). All relevant features, by definition, are present in all examples of a concept. When only examples are shown, 
individuals can classify any feature that remains constant as a relevant feature. However, not all features that are constant across examples must be relevant features. Nonexamples help individuals to learn which features are relevant (i.e., features that, when changed, cause the example to become a nonexample). For instance, teaching the concept square, without nonexamples, could also just teach responding "square" in the presence of any rectangle because all squares are rectangles. It is necessary to show nonexamples of rectangles that are not squares to specify that four sides of equal length is a relevant feature. Without sufficient nonexamples, individuals may not learn to discriminate between the presence and absence of each relevant feature. Without learning this discrimination, when shown an untrained stimulus lacking one or more relevant features, individuals may erroneously label the stimulus as an example of a concept (i.e., overgeneralization; Markle \& Tiemann, 1970a; Tennyson et al., 1972; Woolley \& Tennyson, 1972).

Although Shumway and Lester (1974) demonstrated the importance of including nonexamples while teaching concepts, they did not describe how nonexamples should be selected. The selection of nonexamples to use during instruction may be particularly important when the relevant and irrelevant features of a concept cannot be communicated verbally (e.g., teaching young students or students with disabilities). Some nonexamples may more effectively teach a concept than others, depending on the relation between the examples and nonexamples. For instance, concept formation may be improved when nonexamples that are relatively similar to examples are used (e.g., square and triangle) compared to nonexamples that are relatively dissimilar (e.g., square and car). The relation between examples and nonexamples can be described in terms of two attributes: the number of relevant (e.g., number of sides) and irrelevant features (e.g., color) that an example and nonexample have in common. 
Woolley and Tennyson (1972) recommend using examples and nonexamples with identical irrelevant features (matched examples and nonexamples); matched examples and nonexamples share irrelevant features. For instance, a square has three relevant features: (a) four lines each intersect with two other lines, (b) lines intersect at right angles, (c) lines of equal length. If the concept "square" was being taught, a matched example-nonexample pair could consist of a square and triangle that share the irrelevant features of color, orientation, and size. Matching these irrelevant features ensures the only differences between the square and triangle are the presence or absence of relevant features (e.g., that the square has four sides of equal length and the triangle does not). Various examples and nonexamples are shown in Figure 1. In the first column of Figure 1, there are three examples of squares because each has all three relevant features, even though the color, orientation, and size differ across these examples (irrelevant features). The middle and right columns show two nonexamples with irrelevant features matched with each example of a square; all geometric figures in each row are the same color, orientation, and size as the example to which they are matched.

Despite having similar irrelevant features, the nonexamples have had at least one relevant feature replaced

\section{Figure 1}

Depictions of three examples of the concept "square" and two nonexamples with irrelevant features matched with each example of a square.

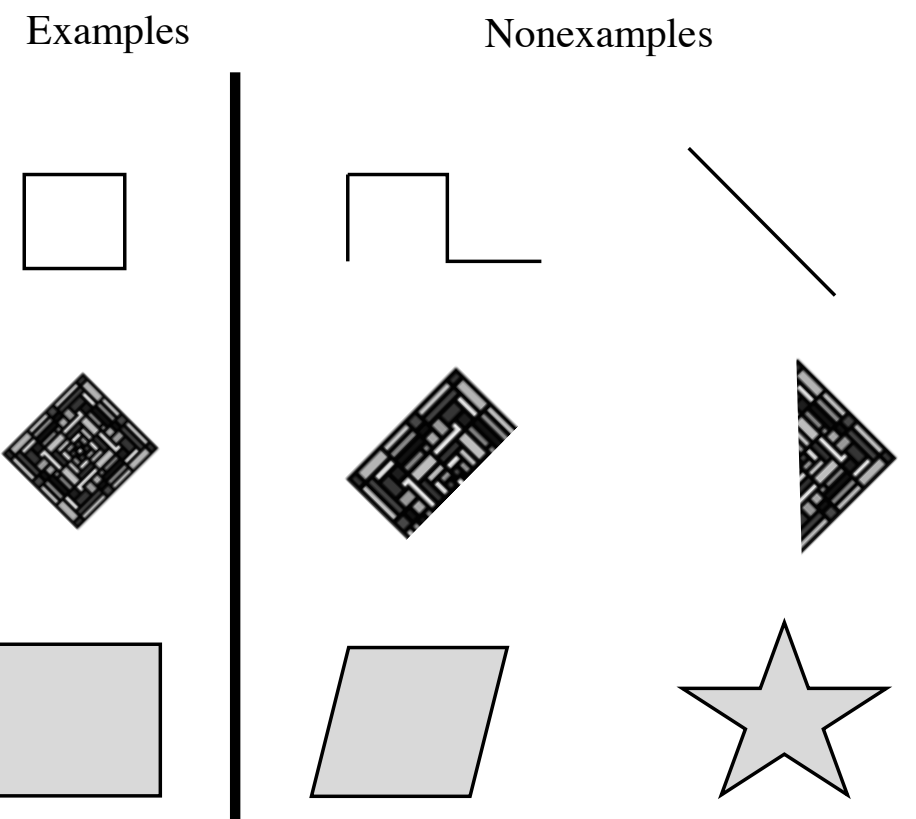


with another feature incompatible with the relevant feature (i.e., replacement feature; e.g., line that intercepts with fewer than two lines, lines that do not intercept at right angles, or sides of different lengths), making them nonexamples of a square.

Experiments have compared concept formation between students (in primary, secondary, and post-secondary education) who were taught using matched and unmatched nonexamples (Merrill \& Tennyson, 1978; Tennyson et al., 1972; Tennyson, 1973). In each of these experiments, the students were taught a set of concepts and then given a test that required them to categorize untrained examples and nonexamples. Regardless of the concept, students taught using matched examples and nonexamples correctly identified untrained stimuli as nonexamples more often than students trained using unmatched examples and nonexamples: Matching the irrelevant features of examples and nonexamples improved concept formation.

Less is known about effects of the number of relevant features that differ between examples and nonexamples. No previous research (to my knowledge) has varied the number of relevant features present in the nonexamples used during training. None of the previous investigations on matched examples and nonexamples (Merrill \& Tennyson, 1978; Tennyson et al., 1972; Tennyson, 1973) described how many relevant features differed between the pairs of examples and nonexamples. The definition of matched examples and nonexamples does not consistently specify how many relevant features should differ between matched examples and nonexamples. Wooley and Tennyson (1972) wrote that matched examples and nonexamples can differ by more than one relevant feature, but Markle (1990) specified that matched nonexamples must lack exactly one relevant feature to be matched to an example. It is unknown how the number of relevant features missing from nonexamples affects concept formation. 
The number of missing relevant features changes how different examples and nonexamples are from each other. Nonexamples can be categorized according to the number of missing relevant features

(Markle, 1990, Markle \&

Figure 2

Depictions of Examples, Close-In Nonexamples, and

Tiemann, 1970a, Woolley \& Far-Out Nonexamples of the Concept "Square"

Tennyson, 1972). Far-out

nonexamples lack more relevant

features (i.e., differ more from

examples) than close-in

nonexamples. Figure 2 shows

three examples of the concept

"square" and two nonexamples

with the same irrelevant features

as each example. Note that only

one relevant feature differs
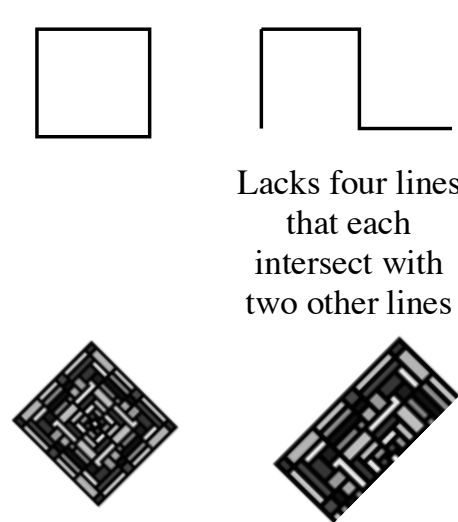

Lacks four lines that each intersect with two other lines

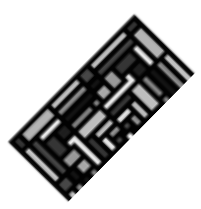

Lacks lines of equal length
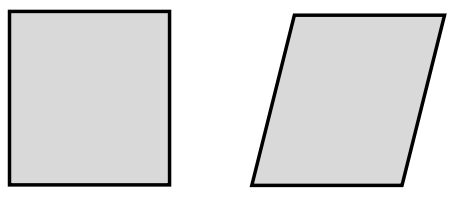

Lacks lines that intersect at right angles

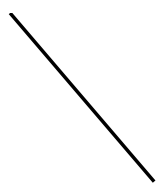

Lacks all relevant features

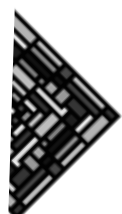

Lacks all relevant features

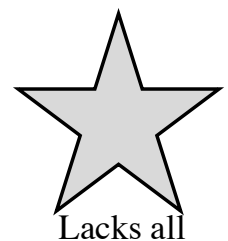

relevant features

between each example and the

nonexample in the second

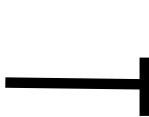

0

(Example)

(Close-In Nonexample)

column (they are close-in

\# Relevant Features Missing

nonexamples). The missing relevant feature has been replaced with a replacement feature and all other relevant features are present. If the three pairs of examples and close-in nonexamples were used to teach the concept "square", the individual would have to learn each relevant feature to answer correctly during training. In contrast, all three relevant features differ between each example and the nonexample in the third column (they are far-out nonexamples). 
Using far-out or close-in nonexamples may affect how concepts are learned. In fact, previous studies of stimulus categorization suggest that similar and dissimilar stimuli may be processed in different areas of the brain (e.g., Seger et al., 2015, Broschard et al., 2019). Because far-out nonexamples differ from examples in more ways than close-in nonexamples, it may be easier to discriminate between far-out nonexamples and examples, resulting in more correct answers and faster learning. However, to correctly identify examples during training with examples and far-out nonexamples, the individual only needs to learn one relevant feature. If only one relevant feature is learned, responding may not be accurate on tests when untrained examples and nonexamples are presented that share one or more relevant features, indicating a failure in concept formation. It is not clear whether far-out or close-in nonexamples should be used during training when the desired outcome is to complete training as fast as possible while also ensuring concept formation occurs.

The purpose of the present study was to investigate how using nonexamples with different numbers of missing relevant features affects concept formation. Two experiments were conducted to compare concept formation following training with three different conditions. Training in each condition included a different combination of examples and nonexamples: examples only (no nonexamples), examples and close-in nonexamples, and examples and far-out nonexamples. Specifically, all far-out nonexamples in the present investigation lacked all (three) relevant features and all close-in nonexamples lacked exactly one relevant feature. In both experiments, adult participants completed baseline, training, and testing in all three conditions using a computer task. In the first experiment, participants were taught three arbitrary, geometric concepts to mitigate effects of previous learning histories on concept formation. In the second experiment, different participants were taught three biological concepts, akin to those that may 
be taught in a classroom. Differences in concept formation across conditions and experiments were evaluated to demonstrate the role of close-in and far-out nonexamples in concept formation and inform the use of nonexamples to teach concepts.

\section{Experiment 1}

\section{Method}

\section{Participants}

Adults were recruited using a system provided by the university and flyers posted in the community. Participation was not limited by race, ethnicity, gender, or age (as long as the person was at least 18 years old). The demographics for the eight participants enrolled for the experiment, including two who did not complete the experiment, are shown in Table 1. Of the two participants that did not complete the experiment, one was dismissed after she did not attend an appointment for two weeks and one withdrew because participation did not earn extra credit toward a course-grade. Six participants completed all three conditions of the experiment (bolded in Table 1). The average age of these participants was 19.50 years. At the start of the first appointment with a potential participant, the experimenter reviewed a consent form (see Appendix A) that described the experimental task, monetary incentives, and the right to withdraw from the experiment at any time. The consent form was approved by the university's Institutional Review Board for the Protection of Human Subjects. After signing the consent form, the participant completed a brief demographics survey (see Appendix B) and started the experimental task.

Each participant agreed to attend two to five appointments per week, with each appointment lasting approximately $45 \mathrm{~min}$ and scheduled at about the same time each day. The number of appointments scheduled each week was the same for each participant every week but 
varied across participants (shown in Table 1). Appointments ended when a block of trials was completed after at least $40 \mathrm{~min}$ had elapsed from the start of the appointment (to prevent the appointment from ending in the middle of a block) or after training or testing was completed in a condition. Appointments were scheduled until the participant completed all three training conditions, withdrew from the experiment, or completed 20 total hours of sessions.

Participants earned $\$ 0.04$ for each correct response they made on training trials and $\$ 5.00$ for each set of baseline and test blocks in each condition ( $\$ 15.00$ total across all three conditions); the participants were given a daily total earnings statement at the end of the day's appointment. If the participant withdrew before completing the experiment, they were paid what they earned to that point. After their last appointment, the participant was given a prepaid MasterCard loaded with the total amount of money earned. Cards for participants who completed the experiment also included a bonus equal to $25 \%$ of their earnings for correct responses. The amount of money earned by each participant is shown in Table 1. Participant 101's and 104's (both of whom did not complete the experiment) total earnings averaged less than $\$ 7.00$ per hour, so supplementary earnings were added to increase the hourly earnings to $\$ 7.00$ an hour.

\section{Setting and Apparatus}

Each participant worked alone in a 4.1-m by 3-m room equipped with a one-way mirror that allowed the experimenter to make unobtrusive observations. Before each appointment, the participant left all personal belongings in a separate secure area.

The experimental task was presented using a Visual Basic ${ }^{\circledR}$ program on a Microsoft Surface 3 tablet with a touch screen (27.5-cm display; 2160-pixel x 1440-pixel resolution) or Nextbook Flexx 11 tablet with a touch screen (29.5-cm display; 1366-pixel x 768-pixel 
Table 1

Experiment 1 Participants

\begin{tabular}{llccccccccc}
$\begin{array}{l}\text { ID } \\
\#\end{array}$ & Gender & Age & Ethnicity & Student? & Outcome & $\begin{array}{c}\text { Appointments } \\
\text { /Week }\end{array}$ & $\begin{array}{c}\text { Time in } \\
\text { Appointments }\end{array}$ & $\begin{array}{c}\text { Experiment } \\
\text { (hr) }\end{array}$ & $\begin{array}{c}\$ \\
\text { Earned }\end{array}$ & $\begin{array}{c}\text { Earned/ } \\
\text { Hour }\end{array}$ \\
\hline 101 & Female & 28 & White & No & Dismissed & 3 & 1 & 0.70 & 2.50 & 3.57 \\
$\mathbf{1 0 2}$ & Female & $\mathbf{1 8}$ & White & Yes & Completed & $\mathbf{2}$ & $\mathbf{7}$ & $\mathbf{2 . 7 5}$ & $\mathbf{3 2 . 3 5}$ & $\mathbf{1 1 . 7 6}$ \\
$\mathbf{1 0 3}$ & Female & $\mathbf{1 9}$ & White & Yes & Completed & $\mathbf{3}$ & $\mathbf{8}$ & $\mathbf{3 . 8 9}$ & $\mathbf{3 8 . 2 5}$ & $\mathbf{9 . 8 3}$ \\
104 & Female & 18 & - & Yes & Withdrew & 3 & 5 & 2.82 & 14.48 & 5.13 \\
$\mathbf{1 0 5}$ & Female & $\mathbf{1 8}$ & White & Yes & Completed & $\mathbf{2}$ & $\mathbf{8}$ & $\mathbf{3 . 1 9}$ & $\mathbf{3 7 . 6 6}$ & $\mathbf{4 7 . 0 8}$ \\
$\mathbf{1 0 6}$ & Female & $\mathbf{2 0}$ & Asian & Yes & Completed & $\mathbf{3}$ & $\mathbf{6}$ & $\mathbf{2 . 8 3}$ & $\mathbf{3 2 . 3 0}$ & $\mathbf{1 1 . 4 1}$ \\
$\mathbf{1 0 7}$ & Male & $\mathbf{1 8}$ & White & Yes & Completed & $\mathbf{3}$ & $\mathbf{9}$ & $\mathbf{3 . 2 7}$ & $\mathbf{5 3 . 3 0}$ & $\mathbf{1 6 . 3 0}$ \\
$\mathbf{1 0 8}$ & Female & $\mathbf{2 4}$ & Black & Yes & Completed & $\mathbf{4}$ & $\mathbf{1 4}$ & $\mathbf{7 . 5 1}$ & $\mathbf{5 5 . 1 5}$ & $\mathbf{7 . 3 4}$
\end{tabular}


resolution). Sounds were played using the tablets' native speakers set to the $50 \%$ volume setting (about $10 \mathrm{~dB}$ measured $90 \mathrm{~cm}$ from the touch screen). All responses were made by touching the screen. If, at any point, the participant did not make a response within $1 \mathrm{~min}$, all stimuli and text on the screen disappeared, and a reminder box appeared in the middle of the screen that said "Please respond as quickly as you can" with a button below containing text that said "ok". When "ok" was touched, the same stimuli and text reappeared, and the trial timer resumed. Unless noted otherwise, all text displayed by the program was in black, Microsoft Sans Serif 24-point font.

\section{Experimental Design}

A different concept was taught in each of three training conditions (Examples Only, Close-In Nonexamples, and Far-Out Nonexamples). The concept assigned to each condition was the same across participants. Hereafter, each concept will be referred to in terms of the condition in which it was taught (e.g., the concept taught in the Examples-Only condition will be called the Examples-Only concept). Conditions were signaled by the color of the experimental stimuli (grey, blue, or yellow), and the order of the colors remained constant across participants. The six participants were randomly assigned without replacement to one of the six possible combinations of color and condition, so that the color assigned to each condition was counterbalanced across participants. Participants completed baseline, training, and testing in one condition (all stimuli the same color) before beginning baseline in the next condition.

Within each condition, appointments were broken into blocks of 24 trials for the purpose of data analysis, but there was no indication to the participant of the end of one block and the beginning of the next. The number of appointments needed to complete a condition varied depending on the participant's performance. 
In all training conditions, four stimuli were presented in each trial. The number of training stimuli that were examples and nonexamples of the concept varied by condition. In the Examples-Only condition, four examples of the concept were presented in each training trial. In the Close-In Nonexamples condition, one example and three close-in nonexamples of the concept were presented. In the Far-Out Nonexamples condition, one example and three far-out nonexamples of the concept were presented. A single set of 24 stimuli was used during training across all conditions. In the Examples-Only condition, all 24 stimuli were examples. In the Close-In Nonexamples condition, six stimuli were examples and 18 were close-in nonexamples. In the Far-Out Nonexamples condition, six stimuli were examples and 18 were far-out nonexamples.

\section{Trial Structure}

At the start of each trial, a white rectangle (116 by 42 pixels, approximately 3.0 by 1.1 $\mathrm{cm}$ ) appeared with the word "START" printed on it in black (20 point). When the participant touched the rectangle, it disappeared and four stimuli appeared on the white background, one in each corner of the screen. Each stimulus was 250 by 250 pixels (approximately 6.6 by $6.6 \mathrm{~cm}$ ). The center of the stimulus was located $0.5 \mathrm{~cm}$ away from the two sides of the screen that defined the corner.

The stimuli remained on the screen until the participant selected one (by touching it). When a stimulus was selected, all stimuli disappeared, and written feedback appeared in the center of the screen. Feedback was positive (reinforcement), negative (punishment), or neutral depending on the selection and condition. In training trials, positive or negative feedback was provided when an example was selected (correct responses) or a nonexample was selected (incorrect response), respectively. In baseline and test trials, neutral feedback followed all 
responses (both correct and incorrect). Positive feedback consisted of text that said "Right! + $\$ 0.04$ " and a concurrent trumpet fanfare played for $1 \mathrm{~s}$. During the fanfare, the total amount of money earned so far in the experiment was displayed for $0.25 \mathrm{~s}$, disappeared for $0.25 \mathrm{~s}$, and reappeared for $0.5 \mathrm{~s}$ with $\$ 0.04$ added to the total. Neutral feedback consisted of text that said "Selected"; no auditory stimulus was played, and the total amount of money earned was not shown. Negative feedback consisted of text that said "Wrong. - \$0.04" and a concurrent buzzer sound that was played for $1 \mathrm{~s}$. During the buzzer, the total amount of money earned so far in the experiment was displayed for $0.25 \mathrm{~s}$, disappeared for $0.25 \mathrm{~s}$, and reappeared for $0.5 \mathrm{~s}$ with $\$ 0.04$ subtracted from the total. The total never went below $\$ 0.00$. All feedback stimuli ended after $1 \mathrm{~s}$, followed by a 1-s intertrial interval during which a blank, white screen was shown. At the end of the intertrial interval, the next trial (or, following an incorrect response during training, a correction trial) began. Correction trials contained exactly the same four stimuli as the previous trial, but the stimuli were randomly reassigned to the corners of the screen. Though the number of correction trials was recorded, correction trials did not count as one of the 24 trials in a block, nor were they included in summary data analyses for that block.

\section{Experimental Stimuli}

Each stimulus had nine features; three were relevant features of the concept to be taught. A stimulus with all three relevant features was an example of the concept, a stimulus with just two relevant features was a close-in nonexample, and a stimulus with no relevant features was a far-out nonexample. Figure 3 shows the relevant features of the Examples-Only, Close-In Nonexamples, and Far-Out Nonexamples concepts in the top, middle, and bottom row, respectively. The name of the relevant feature shown is printed below each stimulus. All stimuli (examples, close-in nonexamples, and far-out nonexamples) had the same number of lines 
(three), geometric objects

(three), and geometric-object

fills (three). The relevant

features of each concept

were a particular line

(inside/outside line, inside

line, or outside line), a

particular geometric object

(rectangle, triangle, or oval),

and a particular geometric-

object fill (horizontal line

through the top-center,

bottom-left, or bottom-right

geometric figures). For
Figure 3

Each of the Three Relevant Features of Each Concept and an Example of Each Experiment 1 Concept

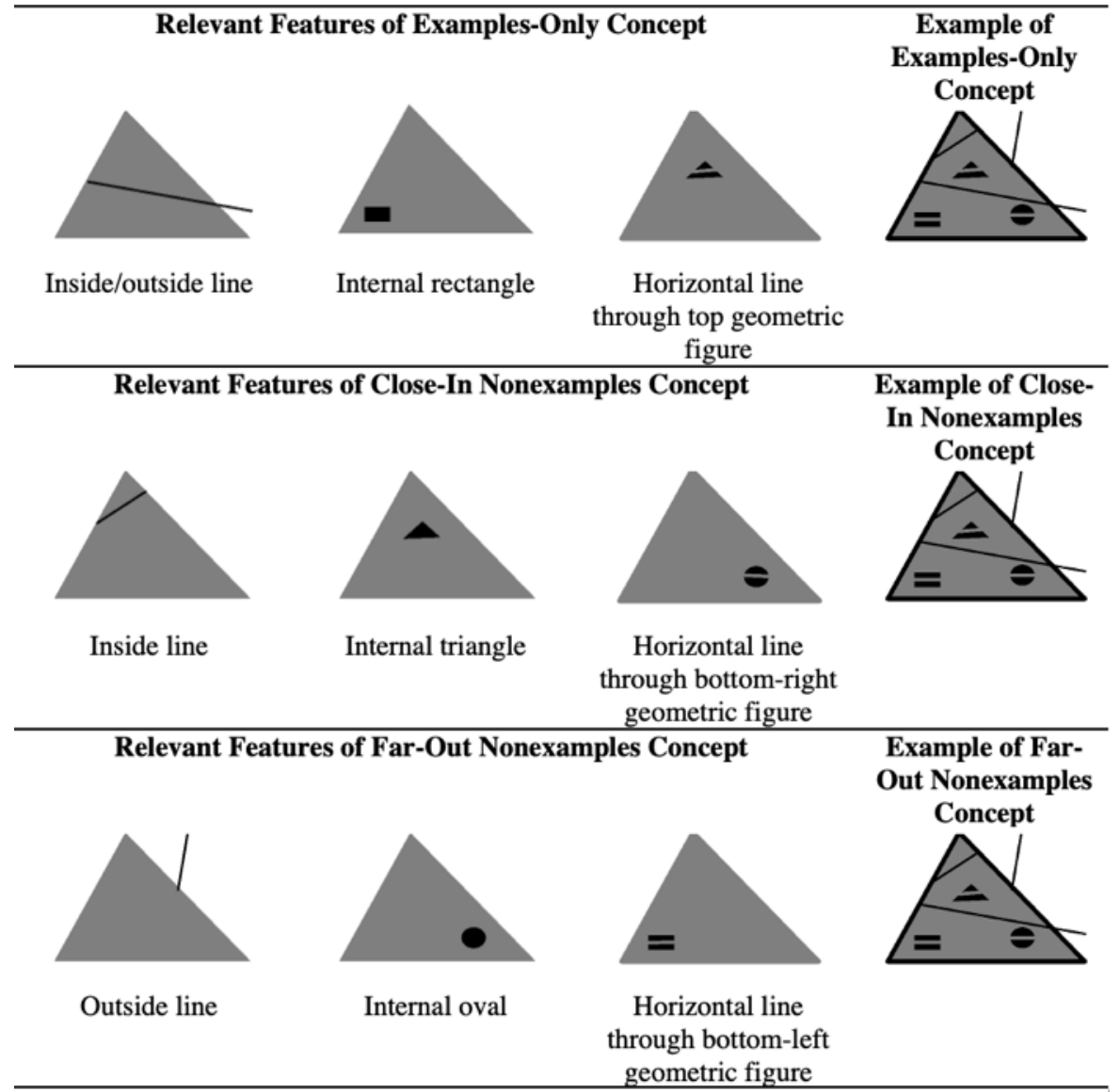

instance, examples of the Examples-Only concept had an inside and outside line (line that intersects two sides and crosses the side), a rectangle, and a horizontal line through the top-center geometric figure (in the case of Figure 3, a triangle).

The right-most stimulus in each row of Figure 3 is an example of each concept. Note that, in the case of this figure, the same stimulus is able to be an example for all three concepts, but the three features that make it an example differ for each concept. In the case of the example stimuli in the right column of Figure 3, the six features that were not relevant for each concept (i.e., irrelevant features) served as relevant features for the other two concepts. Alternatively, a stimulus (not shown in Figure 3) could have had all three features for only zero, one, or two 
concepts, while lacking at least one relevant feature of other concepts. This means that a stimulus could have been a nonexample of a given concept(s) while being an example, close-in nonexample, or far-out nonexample of other concept(s), allowing the same set of stimuli to be used in training and testing in all conditions.

Figure 4 demonstrates how a single stimulus could be an example, close-in nonexample, or far-out nonexample for any combination of concepts. The names of the relevant features that are present are printed below each stimulus. The triangles in each row are identical, yet a stimulus in one row may be an example of one concept, while the identical stimulus in another row may be a nonexample for another concept. For instance, the left-most triangles contain all three
Figure 4

Example, Close-In Nonexample, and Far-Out Nonexample of Each Concept

Examples-
Only
Concept
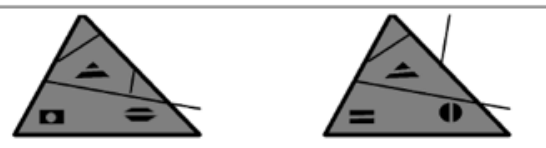

Examples

1.Inside/outside line

2.Internal rectangle 3.Horizontal line through top geometric figure

Close-In
Nonexamples
Concept
Concept

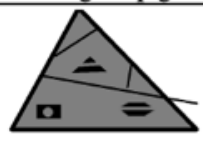

Example

Close-In Nonexample

1. ---

2. Internal triangle

3. Horizontal line through bottom-right geometric figure

Far-Out
Nonexamples
Concept

Far-Out Nonexample 1. ---

2. ---

3. ---
1. Inside line

2. Internal triangle

3. Horizontal line through bottom-right geometric figure

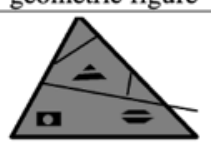

Far-Out Nonexample 1. ---

2. ---

3. ---

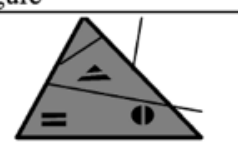

Close-In Nonexample

1. Inside line

2. Internal triangle

3. ---

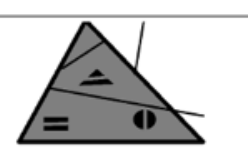

Example

1. Outside line

2. Internal oval

3. Horizontal line through bottom-left geometric figure

relevant features of the Examples-Only concept (making it an example of this concept, as would be shown for training in that condition), two of the three relevant features of the Close-In Nonexamples concept (making it a close-in nonexample of this concept), and zero of the three relevant of the Far-Out Nonexamples condition (making it a far-out nonexample of this concept). Similarly, the middle triangles contain all three relevant features of both the Examples-Only and 
Close-In Nonexamples concepts (making it an example of both these concepts) but lack all three relevant features of the Far-Out Nonexamples concept (making it a far-out nonexample of this concept). The right-most triangles contain all three relevant features of both the Examples-Only and Far-Out Nonexamples concepts (making it an example of these concepts) but lack a relevant feature of the Close-In Nonexamples concept (making it a close-in nonexample of this concept).

Whenever a stimulus is missing a relevant feature for any concept, that feature was replaced with a replacement feature that was irrelevant for all concepts so that the number of features in each stimulus (i.e., stimulus complexity) remained constant. Replacement features were a line (line touching no sides, line touching one side and going in, line going through one side, but not touching any other side), geometric object (hexagon or star), or a fill pattern (vertical line or circle). All stimuli in Experiment 1 are shown in Appendix C. Pre-experimental evaluations related to the development of these stimuli have been summarized in Appendix D.

\section{Procedure}

The following procedures were informed by pilot tests done prior to the experiment proper. Changes between the pilot testing procedure and the current procedure are summarized in Appendix E.

Baseline. Appointments at the start of each condition began with six baseline blocks of 24 trials. All responses during baseline trials were followed by neutral feedback. Responding during baseline was compared to responding on testing trials after training in each condition.

The following instructions appeared in text in the top, center of the screen before the first trial of each baseline began:

You will be shown four images at a time. Your job is to select an image you think is right. The images are different today, meaning that you may have to answer differently 
now to earn money. For now, you will not be told whether your selection is right or wrong. You will earn $\$ 2.50$ for selecting answers. When you are done, a message will appear giving you instructions for the next part of the study, when you will learn how to identify the correct answers.

The start button (identical to the one that appears at the start of each training trial) was placed directly below this text; the first baseline trial began when the start button was touched.

Baseline and test blocks were arranged in the same way across all training conditions. The stimuli during baseline blocks were identical to the stimuli used in test blocks. A set of 72 stimuli were used during these blocks: the 24 stimuli that were used in training (i.e., training stimuli) and 48 stimuli that were only used again during testing (to assess generalization and discrimination; i.e., untrained stimuli). Baseline and testing blocks contained 24 trials, 12 of which were identical to training trials. During these 12 trials with training stimuli, each of the 24 training stimuli was shown twice.

The other 12 trials each included four stimuli that were not used during training. Of these 48 untrained stimuli, 12 were examples, 18 were close-in nonexamples, and 18 were far-out nonexamples of each concept. Each untrained stimulus appeared exactly once per block. Untrained stimuli were arranged into two trial types (six trials of each type per block); these arrangements were the same across all training conditions. Close-in trials contained one example and three close-in nonexamples. Far-out trials contained one example and three far-out nonexamples. This arrangement allowed for separate measures of responding when the examples and close-in nonexamples were presented (i.e., when examples and nonexamples shared more features) to when the examples and far-out nonexamples were presented (i.e., when examples 
and nonexamples shared more features). Examples of trials with untrained close-in and far-out nonexamples in Experiment 1 are provided in Appendix F.

Training. After the final baseline trial in each condition and at the beginning of all appointments that were not the start of a new condition, text appeared in the top, center of the screen saying:

You will be shown four images at a time. You will earn $\$ .04$ each time you select a correct image and lose $\$ .04$ each time you select an incorrect image. When you select an image, you will be told whether your selection is right or wrong. When you are done, a message will appear on the center of the screen telling you to knock on the door behind you.

Training began when the participant touched the start button. Appendix G shows four stimuli that could be presented in a trial and descriptions of the relation between these stimuli and each training condition. During each block of 24 training trials, each stimulus was shown an equal number of times in each of the four corners on the screen. The proportion of the training stimuli that were examples, close-in nonexamples, and far-out nonexamples of the concept varied across the three conditions but remained constant across training trials within a condition.

A single set of 24 training stimuli was used in all three conditions so that no nonexamples of the Example-Only concept would be shown in training for any condition. Within this set, the number of examples and nonexamples of each concept varied across conditions so that trials with the appropriate number of examples and nonexamples could be arranged. All 24 training stimuli had all three features of the Examples-Only concept. Six of these training stimuli were also examples of the Close-In Nonexamples concept, and the remaining 18 had two of the three relevant features of the Close-In Nonexamples concept (that is, they were close-in nonexamples 
of the Close-In Nonexamples concept). Six of the 24 stimuli were examples of the Far-Out Nonexamples concept, and the remaining 18 had none of the relevant features of the Far-Out Nonexamples concept (that is, they were far-out nonexamples of the Far-Out Nonexamples concept). Training ended after responding was correct in at least 23 of 24 trials (not counting correction trials) for three consecutive blocks or failing to meet this mastery criterion after 25 blocks.

Affect. Immediately following training in each condition, the participant completed the Positive and Negative Affect Scales (PANAS-X; Watson \& Clark, 1994) to determine if some training conditions might elicit different emotional responses than others. This scale (see Appendix H) asked participants to rate (on a five-point Likert scale from Not at all to Extremely) the degree to which each of 60 feelings described how they currently felt. The scale was provided on a single page on Qualtrics.

Testing. The participant was dismissed for the day after completing training in a condition (even if the appointment was shorter than $45 \mathrm{~min}$ ). At the start of the next appointment, the participant completed a single block of training trials to assess maintenance since the previous appointment. If responding was correct for at least 23 of 24 trials, testing began. Otherwise, training continued until the participant responded correctly during at least 23 of 24 trials for three consecutive blocks, at which point the appointment ended. The same process repeated at the start of the next appointment. If a total of 25 blocks of training was completed, testing began regardless of response accuracy.

At the start of testing, text appeared in the top, center of the screen saying "Now you will not be told whether your selection is right or wrong. You will earn $\$ 2.50$ for selecting answers. When you are done, a message will appear on the center of the screen telling you to knock on the 
door behind you." When the participant touched the start button (identical to the one that appeared at the start of each training trial) directly below this text, the testing phase began. Testing consisted of six blocks of test trials. The same stimulus arrangements were used in baseline and testing blocks. All testing for a single condition took place during the same appointment.

\section{Dependent Variables}

For each training and correction trial, the computer program recorded which stimuli were displayed and their locations, the stimulus selected and its location, whether the correct stimulus was selected, whether the trial was a correction trial, and the consequence of the response (positive, negative, or neutral feedback). For each baseline and test trial, the program recorded the trial type (trial with training stimuli, trial with untrained close-in nonexamples, trial with untrained far-out nonexamples) and all of the information recorded on training trials. Responses on the PANAS-X were scored to calculate measures of general negative affect and general positive affect.

\section{Results and Discussion}

The primary purpose of this experiment was to compare concept formation following training with examples, examples and far-out nonexamples, and examples and close-in nonexamples. In each condition, visual inspection was used to compare accuracy across baseline and test trials. Graphs of data from the two participants who did not complete all three conditions of the experiment are shown in Appendix I.

The graphs in Figure 5 show accuracy in the baseline and testing blocks in each condition for Participant 102 and 105. Baseline and testing blocks are separated by a solid, vertical line, indicating when training occurred, but no training data are displayed. Responding on trials with 
training stimuli (triangles in the top graphs) should remain accurate during testing. Indeed, for these two participants, accuracy on trials with training stimuli increased from baseline to testing and was at or near $100 \%$ during testing. The bottom graphs in Figure 5 show accuracy during trials with untrained stimuli. Generally, responding during trials with untrained stimuli remained inaccurate following training in the Examples-Only condition. Responding during trials with untrained examples and far-out nonexamples (squares in the bottom graphs) improved following training in both the Close-In and Far-Out conditions. Responding during trials with untrained examples and close-in nonexamples (circles in the bottom graphs) improved only after training in the Close-In Nonexamples condition. These findings support the hypothesis that, relative to training with only examples, both close-in and far-out nonexamples improved concept formation, with close-in nonexamples improving concept-formation more than far-out nonexamples.

Graphs of data for Participants 103, 107, and 106 are shown in Figure 6 in a manner similar to Figure 5. Unlike Participants 102 and 105, accurate responding was not maintained during test trials with training stimuli in the Close-In Nonexamples condition (103, 107, and 106) and Far-Out Nonexamples condition (106 only), so conclusions about responding in the presence of untrained stimuli must be made with caution. At least two changes between training and testing may have affected responding. First, prior to testing, participants were shown instructions alerting them that they would receive $\$ 2.50$ for completing test trials, regardless of whether they selected the correct response. This may mean that participants were no longer motivated to respond correctly. The motivational differences may have been exacerbated in the Close-In Nonexamples condition because it was more difficult to differentiate examples and nonexamples in this condition. Future research could address this limitation by continuing to pay participants 
Figure 5

Baseline and Testing Accuracy for Participants 102 and 105

102

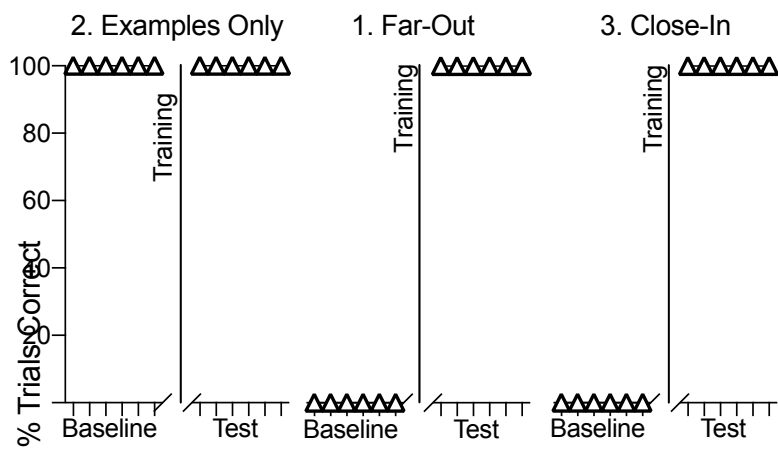

2. Examples Only
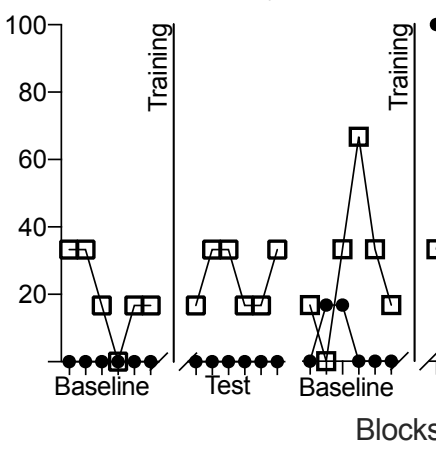

$\triangle$ Training Stimuli

3. Close-In
1. Far-Out

of $\bullet \bullet \bullet \bullet \bullet \bullet$
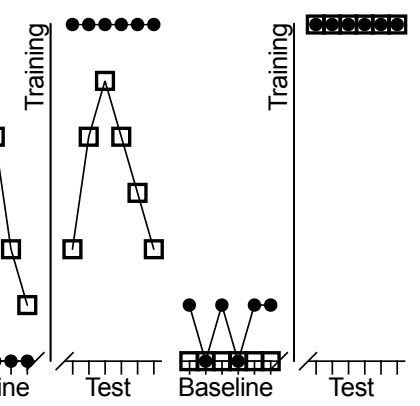

$\rightarrow-$

with Untrained Examples and Close-In Nonexamples
3. Examples Only

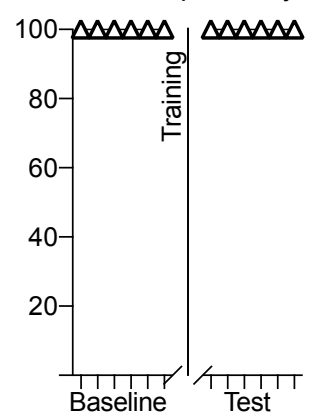

1. Far-Out

2. Close-In
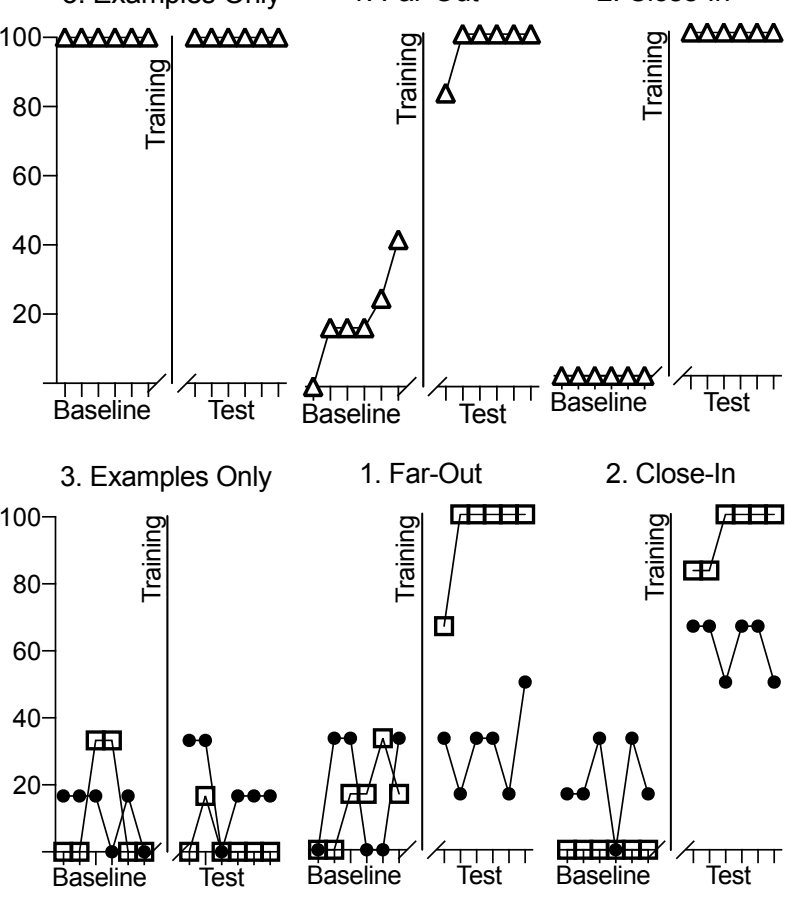

1. Far-Out

2. Close-In

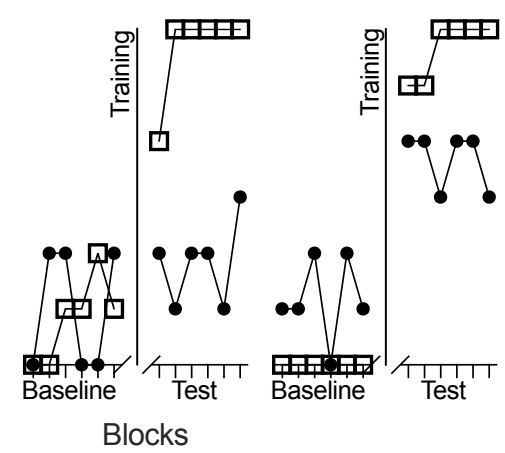

$\square$ Trials with Untrained Examples and Far-Out Nonexamples

Note. Training blocks are indicated by the solid, vertical line between baseline and testing blocks, though data from these blocks are not displayed. The conditions are indicated by the names above the graph and appear in the same order across both participants, though the conditions were not experienced in the same order. The number before the condition name indicates the order in which that condition was experienced. 
based on correct responding during testing or gradually decreasing feedback during training prior to introducing untrained stimuli (e.g., Galizio et al., 2004).

Second, the introduction of untrained stimuli and stimulus combinations (e.g., trials with far-out nonexamples after training that contained only close-in nonexamples) may have affected responding on trials with training stimuli. Responding on trials with training stimuli may be more disrupted when these trials are immediately preceded by trials with untrained stimuli than when they are preceded by trials with training stimuli. Trials with training stimuli and trials with untrained stimuli were randomly dispersed throughout the testing block; thus, the sequence of trials was not controlled. Future research could control the order of trials with untrained and training stimuli.

The lower graphs in Figure 6 show accuracy for Participants 103, 107, and 106 on trials with untrained stimuli during baseline and testing. Across all three participants, accuracy in trials with untrained stimuli remained low following the Examples-Only condition. As might be expected because accuracy on trials with training stimuli was low, accuracy on trials with untrained stimuli was also low for Participants 103, 107, and 106 in the Close-In Nonexamples condition and Participant 107 in the Far-Out Nonexamples condition. Accuracy remained high for Participant 103 in trials with training stimuli in the Far-Out Nonexamples condition but remained low in trials with untrained stimuli. Unlike Participant 103 and 107, responding by Participant 106 on trials with untrained examples and far-out nonexamples improved during testing in both the Far-Out and Close-In Nonexamples conditions (although it improved more in the Far-Out Nonexamples condition). Overall, the results for these three participants demonstrate that, regardless of condition, responding was not accurate on trials with untrained examples and close-in nonexamples if responding on trials with training stimuli was not accurate. 
Figure 6

Baseline and Testing Accuracy for Participants 103, 107, and 106

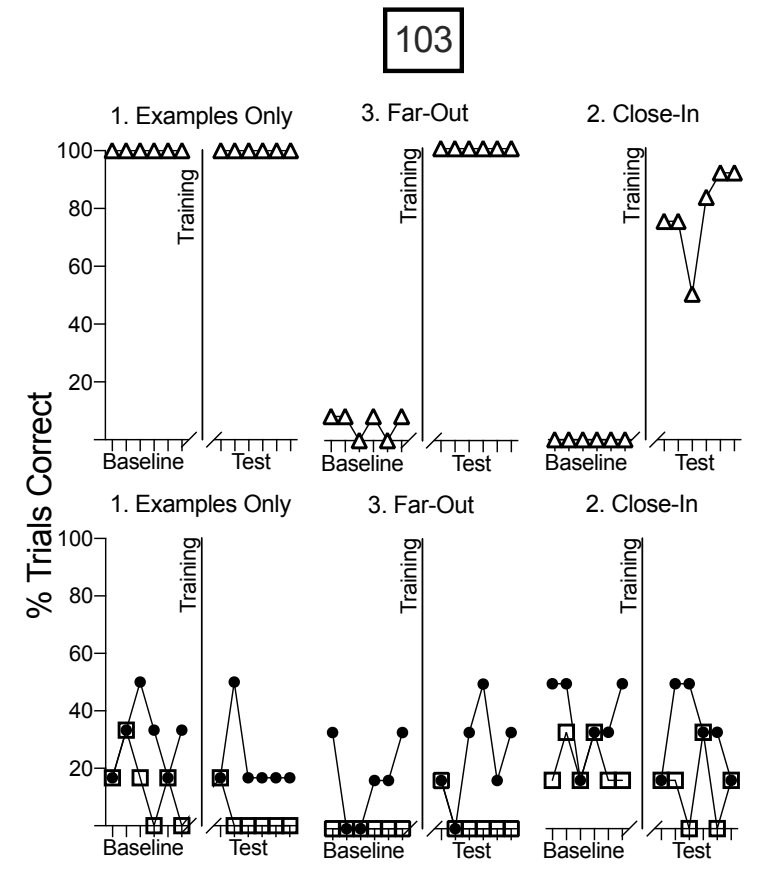

Blocks

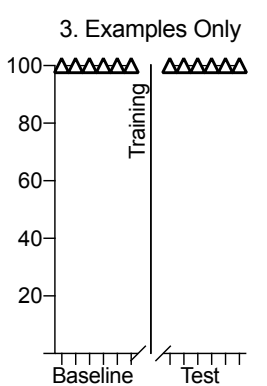

\section{7}
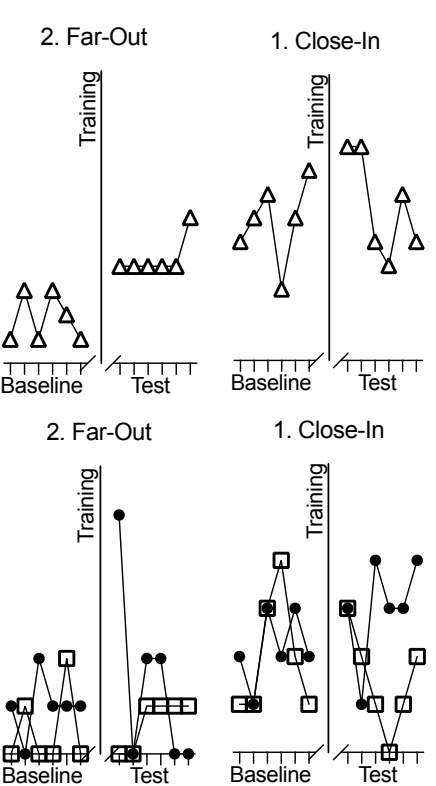

Blocks

$\Delta$ Training Stimuli

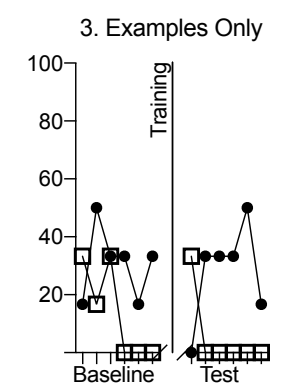

Exampl

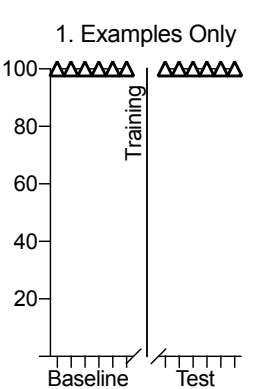

1. Examples Only

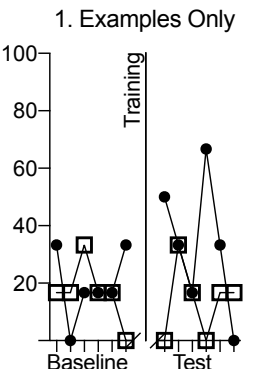

\section{6}

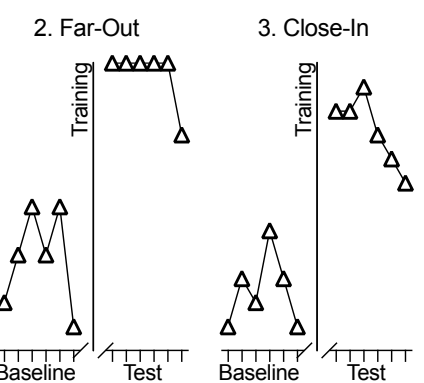

2. Far-Out 3. Close-In

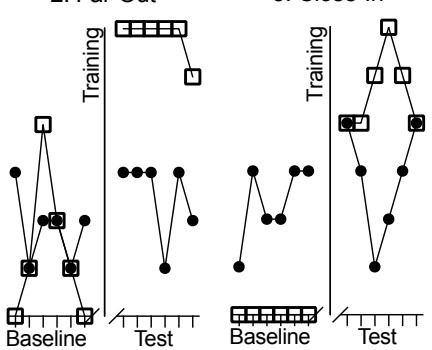

Blocks and Close-In Nonexamples

Trials with Untrained Examples and Far-Out Nonexamples

Note. Training blocks are indicated by the solid, vertical line between baseline and testing blocks, though data from these blocks are not displayed. The conditions are indicated by the names above the graph and appear in the same order across both participants, though the conditions were not experienced in the same order. The number before the condition name indicates the order in which that condition was experienced. 
All of the previously mentioned participants met the training mastery criterion in all conditions prior to testing. However, Participant 108 never responded correctly on more than $42 \%$ of trials in a training block in the Close-In Nonexamples condition that was conducted first (the condition was replicated later, as described below). Training ended because the participant reached the maximum number of training blocks (25). Figure 7 shows graphs of baseline and testing data for Participant 108 in the order that they occurred. As might be expected because responding was never accurate during training, accuracy in the Close-In Nonexamples remained near baseline levels on all test trials (both with training and untrained stimuli). As for all other participants, accuracy remained low on test trials with untrained examples and far-out nonexamples in the Examples-Only condition and untrained examples and close-in nonexamples in both the Examples-Only and Far-Out Nonexamples conditions. Responding was accurate with untrained examples and far-out nonexamples in the Far-Out Nonexamples condition, suggesting discrimination between the presence and absence of at least one relevant feature had been learned.

Discriminating between the presence and absence of one feature (necessary in the FarOut Nonexamples condition) could facilitate learning when it is necessary to discriminate between the presence and absence of multiple features (necessary in the Close-In Nonexamples condition) even though the concepts being taught were different. To see if the completing training in the Far-Out and Examples-Only conditions facilitated learning in the Close-In Nonexamples condition, the Close-In Nonexamples condition was replicated at the end of the experiment. At the start of the replication, the participant was paid the money she earned so far, and the money counter shown during the session was reset to 0 so that the motivation to respond correctly was similar to that in the first Close-In Nonexample condition. In the replication, 
training ended before responding met the accuracy criterion. Learning during trials with close-in nonexamples may have been facilitated by completing training that required easier discriminations (far-out nonexamples), aligning with the general recommendation to begin training with easy discriminations before progressing to more difficult ones (e.g., Fleming \& Levie, 1993; Merrill et al., 1992).

During testing in the Close-In Nonexamples condition replication, responding on trials with training stimuli remained mostly accurate. Responding on trials with untrained examples and far-out nonexamples initially improved relative to baseline but decreased as testing continued. Responding on trials with close-in nonexamples did not improve relative to baseline. It is unclear why conceptual learning was fairly poor following this second training with close-in nonexamples. Disruptions similar to those discussed for Participants 103, 107 and 108 may have occurred between training and testing, though responding on trials with training stimuli did not decrease as much as it did for those participants. Both participants who completed the Close-In Nonexamples condition first (Participant 107 and 108) did not respond accurately on test trials, suggesting that concept formation may not improve as much following only training in the Close-In Nonexamples conditions when previous training conditions had not also been completed, even though different concepts were taught in each condition. Future research could manipulate the order of conditions to identify if particular orders are more likely to produce concept formation than others. 
Figure 7

Baseline and Testing Accuracy for Participants 108

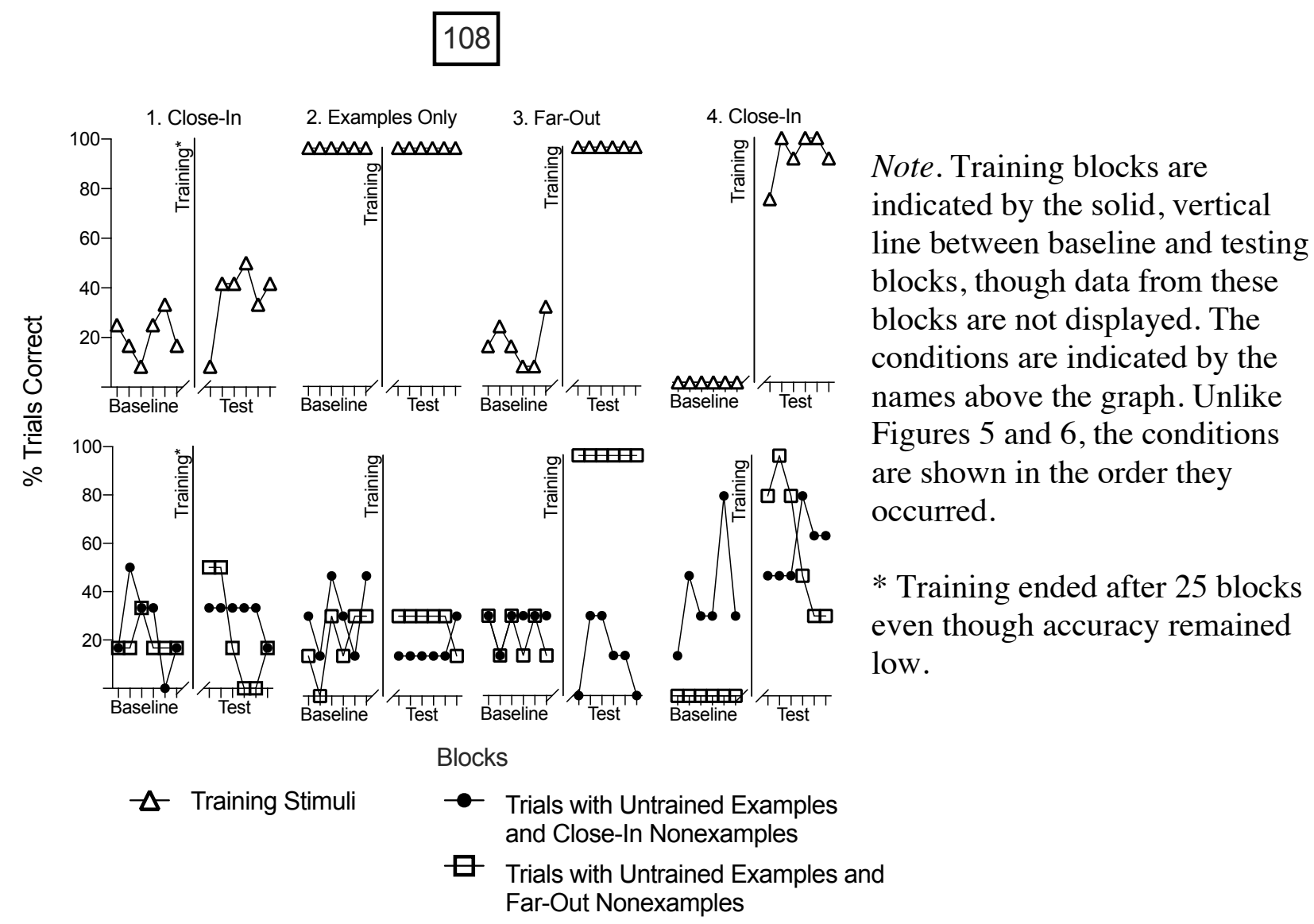


Including close-in nonexamples during training might improve concept formation under some conditions, but training with close-in nonexamples required considerably more time and resources. Figure 8 shows the number of blocks (left) and the number of correction trials (middle) in the Far-Out and Close-In Nonexamples conditions (Participant 108 contributed two data points for the Close-In Nonexamples condition because of the replication of this condition). These variables were compared between the Far-Out and Close-In Nonexamples conditions using a Wilcoxon signed-rank test. Data from the Examples-Only condition were not included because all responses in this condition were correct of necessity; training always ended after the minimum number of blocks (4) and no correction trials. On average, 4.17 more training blocks and 32.00 more correction trials were completed in the Close-In Nonexamples condition than in the Far-Out Nonexamples condition ( $z=-2.20, \mathrm{p}<.05$ for both comparisons $)$.

The right graph in Figure 8 shows the total duration of training in each of the three conditions (including the Examples Only condition). When compared using a Kruskal-Wallis test, the differences in the time to complete training in the Examples-Only $(M d n=8.81 \mathrm{~min})$, FarOut Nonexamples $(M d n=14.77 \mathrm{~min})$, and Close-In Nonexamples $(M d n=57.16 \mathrm{~min})$ conditions were statistically significantly different $(H(2)=14.38, \mathrm{p}<.05)$. A post-hoc Dunn's multiple comparison test indicated a statistically significant difference between the duration of the Examples-Only and Close-In Nonexamples conditions (Mean Rank Difference $=-11.67, \mathrm{p}<.05$ ) but not between the Examples-Only and Far-Out Nonexamples conditions or the Far-Out Nonexamples conditions (Mean Rank Difference $=-4.33, \mathrm{p}=0.55$ ) and Close-In Nonexamples conditions (Mean Rank Difference $=-7.33, \mathrm{p}=0.058$ ). In sum, there were more blocks, time, and incorrect responses during training with close-in nonexamples than during training with examples only or far-out nonexamples. However, whether the larger number of training trials is a 
socially significant difference may depend on the individuals being taught (e.g., tolerance for incorrect responses) and available resources (e.g., amount of available instruction time). Training with far-out nonexamples may be a more efficient approach to concept formation if the learner only needs to learn to discriminate between examples and far-out nonexamples (i.e., they are unlikely to encounter examples and close-in nonexamples outside of the classroom).

Figure 8

Number of Additional Training Blocks, Correction Trials, and Time to Complete Training in each Condition

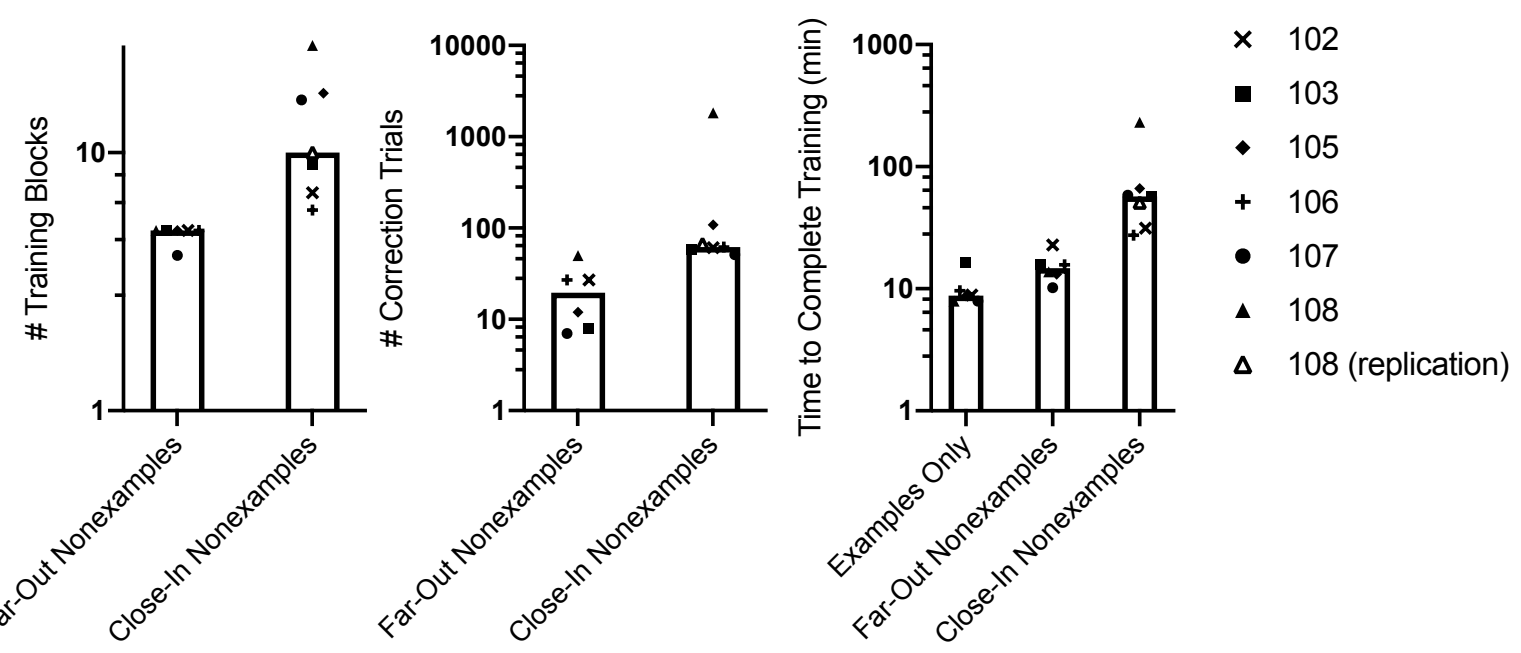

Note. The height of the bar depicts the median and each symbol depicts an individual participant. The y-axis has been scaled logarithmically to account for the large range in values.

As already noted, participants made more incorrect responses in the Close-In Nonexamples condition than in the Far-Out Nonexamples condition. Frequent incorrect responses could result in more negative emotional responding (e.g., Weeks \& Gaylord-Ross, 1981; Zarcone et al., 1994). To evaluate potential emotional effects of each training condition, participants completed the PANAS-X following the third consecutive training block in each condition with correct responses on 23 out of 24 trials. Figure 9 displays the median positive (left graph) and negative (right graph) affect score in each condition, where a higher score indicates 
more positive and negative affect. The median positive-affect score in the Examples-Only, FarOut Nonexamples, and Close-In Nonexamples condition was 24.5, 29.5, and 28, respectively. The median negative-affect score in the Examples-Only, Far-Out Nonexamples, and Close-In Nonexamples condition was $15.5,16$, and 17 , respectively. When compared using a KruskalWallis test, there were no statistically significant differences in positive $(H(2)=0.061, \mathrm{p}=0.97)$ or negative $(H(2)=0.34, \mathrm{p}=0.85)$ affect. These results suggest that the particular training conditions may not have a consistent effect on affect, even when some required involved more errors and take more time. Therefore, effects on affect may not need to be considered as strongly as training resources and the desired training outcomes when selecting the kinds of nonexamples to include during training.

Figure 9

Note. The height of the bar depicts the median, and each symbol depicts an individual participant. A higher score indicates more positive or negative affect, and the highest possible score is 50 .
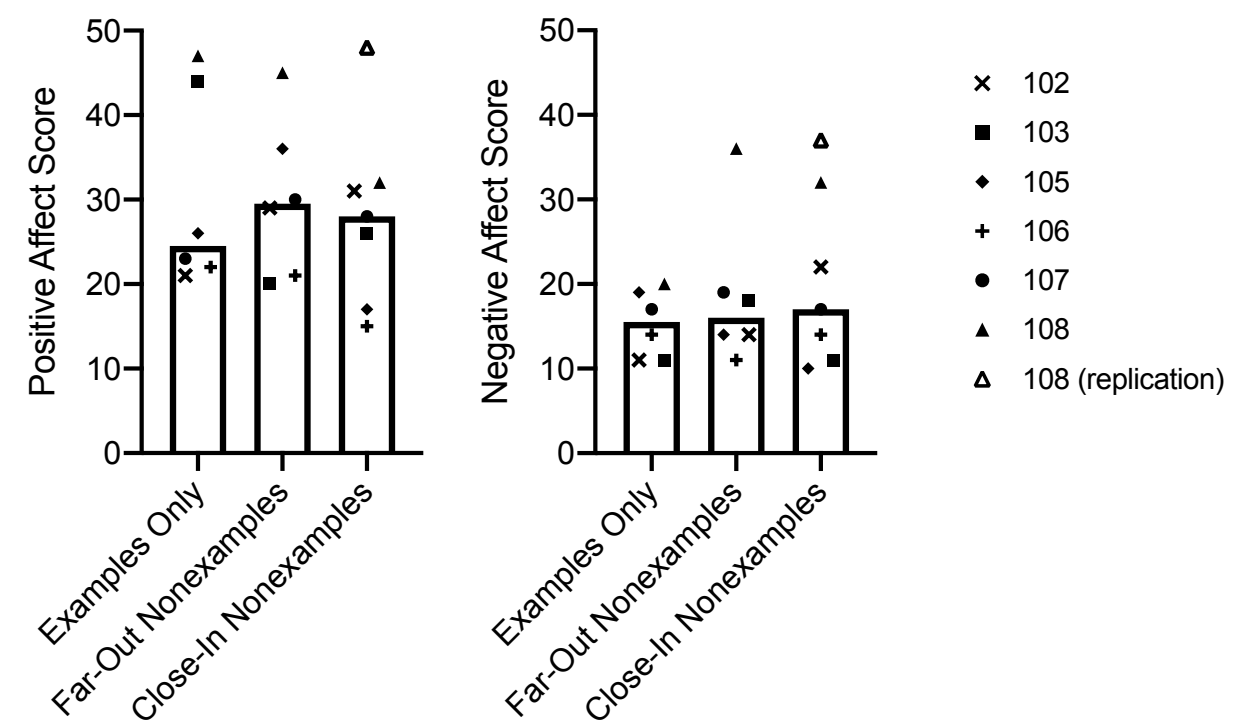

In sum, when responding remained accurate on test trials with training stimuli, nonexamples improved concept formation, with close-in nonexamples improving concept formation more than far-out nonexamples. Although training in the Close-In Nonexamples improved concept formation, accuracy on trials with training stimuli was more likely to decrease and it required additional time and practice relative to training with far-out nonexamples. The 
differences in efficiency between training with close-in and far-out nonexamples may be particularly important when concepts are being taught in the classroom. Ideally, concepts taught in the classroom are related to the previous learning history of the student. Each individuals' knowledge of related concepts could affect how they learn new concepts (e.g., Binder, 1996).

The concepts taught during Experiment 1 were arbitrary shapes that were created to minimize effects of previous knowledge. However, a limitation to using arbitrary concepts is that the results of the study may not generalize to concepts taught in a classroom setting. Experiment 2 investigated effects of nonexamples on concepts that might be taught in a classroom to see if effects of nonexamples changed when non-arbitrary concepts were taught, but no definitions or rules (such as might be typical in a classroom) were provided.

\section{Experiment 2}

\section{Method}

The setting and apparatus, experimental design, and trial structure were identical to those in Experiment 1. Changes between the pilot testing procedure and the current procedure are summarized in Appendix E.

\section{Participants}

Participants were recruited, scheduled, and paid in the same manner as in Experiment 1. The demographics for the twelve participants enrolled for the experiment, including four who did not complete testing in any condition, are shown in Table 2. Of the four participants who did not complete the experiment, two (Participants 206 and 207) were dismissed after responding at a high level of accuracy during the first baseline and two (Participants 203 and 212) withdrew prior to finishing the first condition. 
Table 2

Experiment 2 Participants

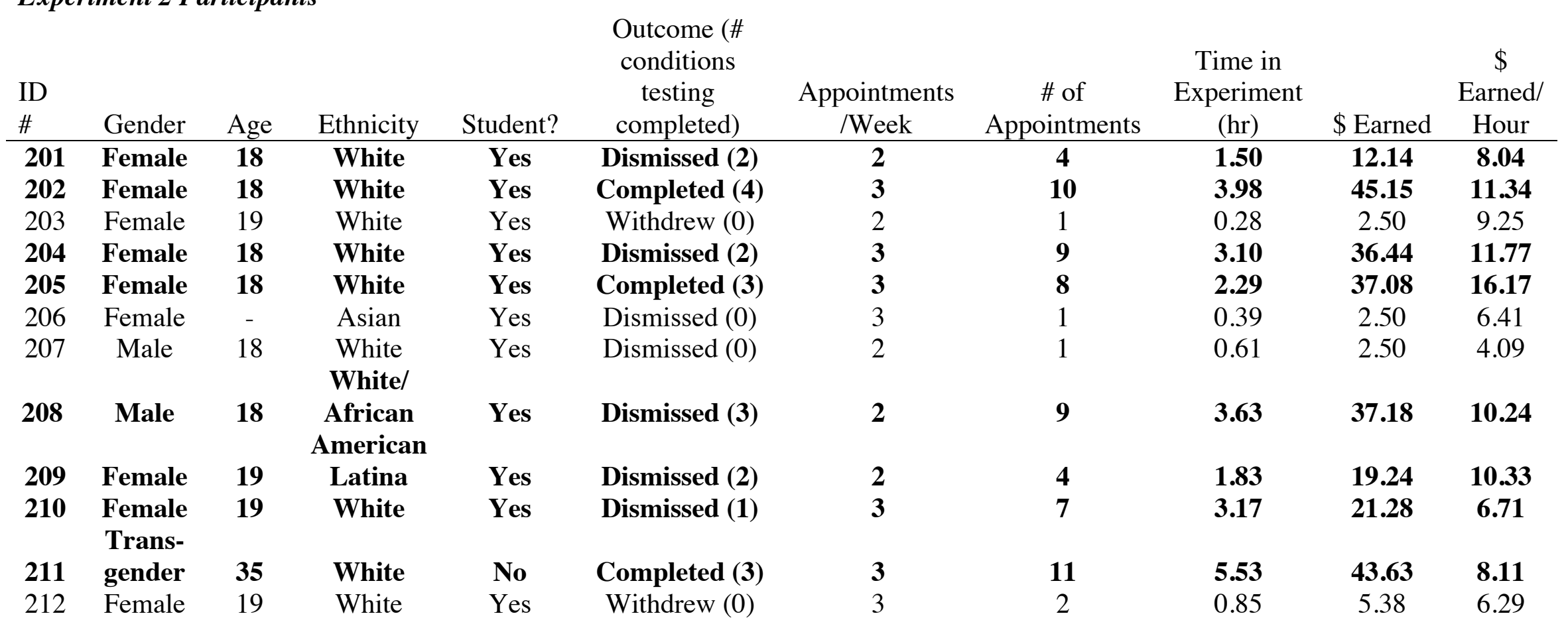


Eight participants completed testing in at least one condition of the experiment (bolded in Table 2). The average age was 20.38 years. The amount of money earned by each participant is shown in Table 2. Participants 206's, 207's, 210's, and 212's (all of whom either withdrew or were dismissed) total earnings averaged less than $\$ 7.00$ per hour, so supplementary earnings were added to increase the hourly earnings to $\$ 7.00$.

\section{Training Stimuli}

One of three biological concepts was taught in each condition: sensory neurons, bacteria, or plant cells. Each biological concept had three relevant features. When a relevant feature was

Figure 10

Each of the Three Relevant Features of Each Examples-Only Concerted an Example of Each Experiment 1 Concept

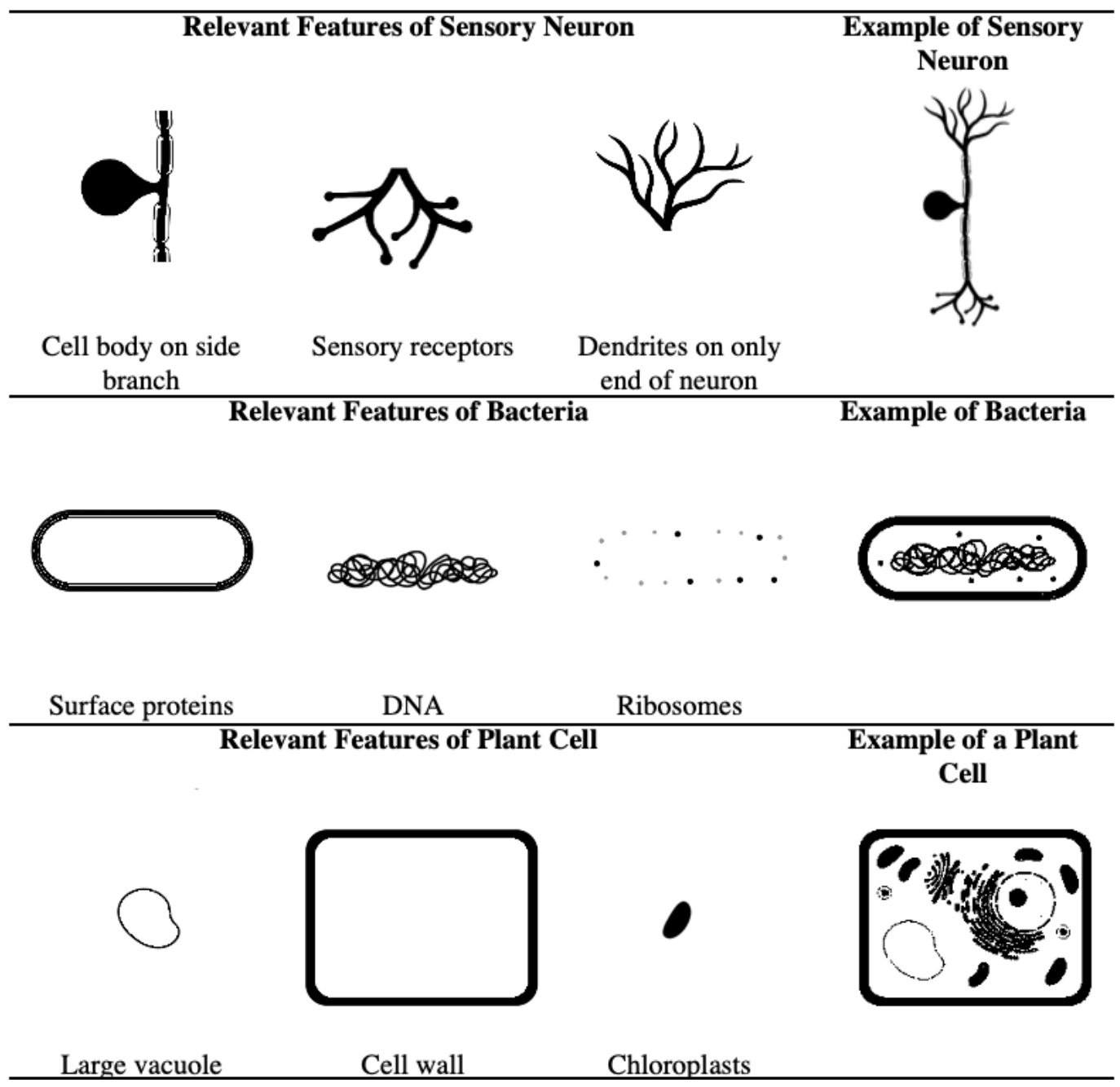


not present, it was replaced with one of two other features so that the overall complexity of the stimulus remained similar across all stimuli. The top row of Figure 10 shows three relevant features of sensory neurons, which are cell body on side branch, sensory receptors, and dendrites on one only end of the neuron. The middle row of Figure 10 shows three relevant features of bacteria, which are surface proteins, DNA, and ribosomes. The bottom row of Figure 10 shows three relevant features of plant cells, which are a large vacuole, cellulose cell wall, and chloroplasts. In addition to the relevant features, all cells had three irrelevant features that varied across stimuli: the cell's orientation, the cell's color scheme, and the location of each feature of the cell. The irrelevant features vary roughly equally across all examples and nonexamples of each concept. All stimuli used in Experiment 2 are shown in Appendix $\mathbf{J}$ and were created with BioRender.com.

Figure 11 shows an example, close-in nonexample (missing one relevant feature), and far-out nonexample (missing all three relevant features) for each concept. As in Experiment 1, Figure 11

Example, Close-In Nonexample, and Far-Out Nonexample of Each Concept in Experiment 2

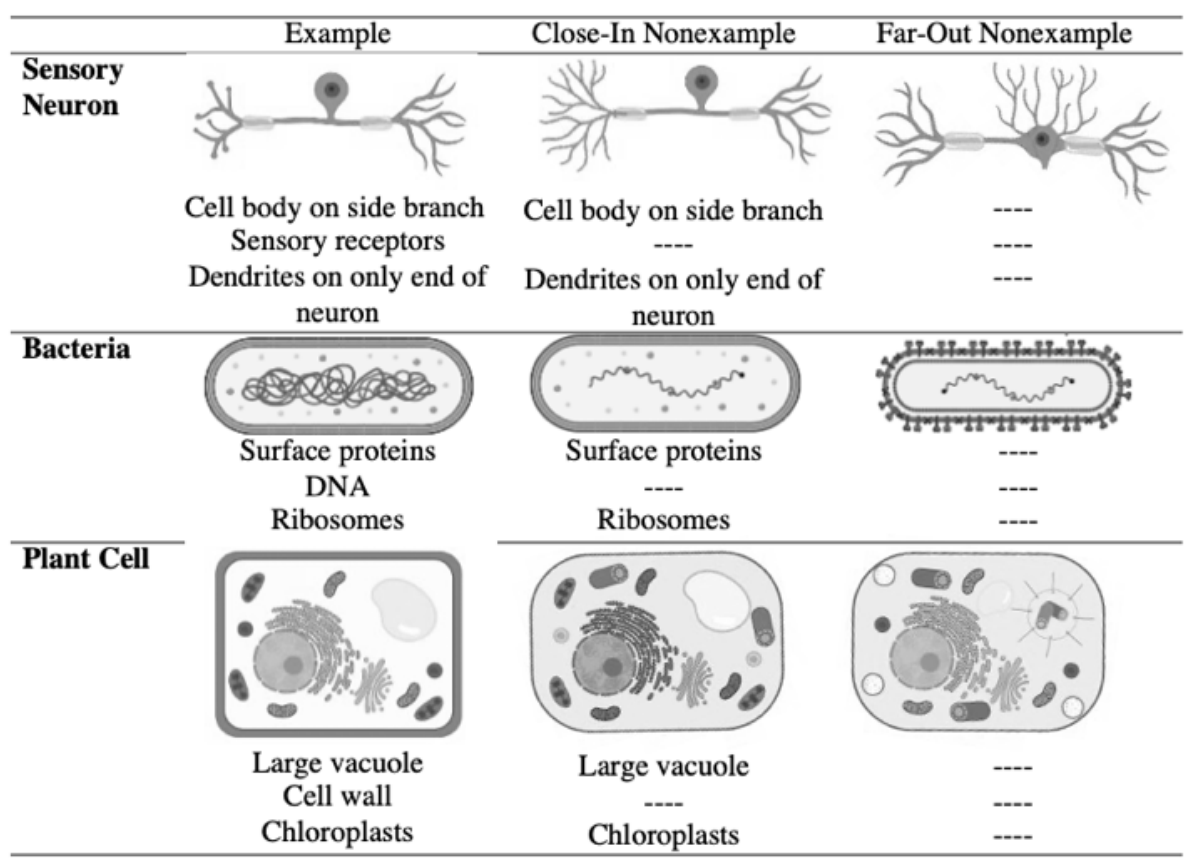


each missing relevant feature was replaced with one of two replacement features to keep the stimulus complexity constant. The irrelevant features of these stimuli have been held constant in Figure 11 to draw attention to the relevant features. The names of the relevant features present are printed below each stimulus.

As in Experiment 1, training trials contained four examples in the Examples-Only condition, one example and three close-in nonexamples in the Close-In Nonexamples condition, and one example and three far-out nonexamples in the Far-Out Nonexamples condition. A different concept was taught in each of the three conditions. A training stimulus set was created for each of the three concepts in each of the three conditions (nine stimulus sets total), but each participant only completed training with three stimulus sets (one set for each condition). The order in which the biological concepts were introduced remained the same across participants, but the order of the three training conditions was counterbalanced across participants as in Experiment 1.

The set of 24 training stimuli used in the Examples-Only condition had all features of the Examples-Only concept. Of the 24 training stimuli used in the Close-In Nonexamples condition, six were examples of the Close-In Nonexamples concept, and the remaining 18 had two of the three relevant features of the Close-In Nonexamples concept (close-in nonexamples of Close-In Nonexamples concept). Of the set of 24 training stimuli used in the Far-Out Nonexamples condition, six of these stimuli were examples of the Far-Out Nonexamples concept, and the remaining 18 had none of the relevant features of the Far-Out Nonexamples concept (far-out nonexamples of Far-Out Nonexamples concept).

Blocks of baseline and test trials were arranged with the stimulus set associated with each biological concept in the same way as in Experiment 1. Examples of trials with untrained close- 
in and far-out nonexamples with each stimulus set in Experiment 2 are provided in Appendix K. Pre-experimental evaluations related to the development of these stimuli have been summarized in Appendix L.

Mid-Experiment Stimulus Modifications. Stimuli that differed from those described above were used at the beginning of Experiment 2 data collection for the first 8 participants (201-208). Each relevant feature was originally replaced with the same nonrelevant feature. However, this resulted in all far-out nonexamples looking similar to each other, so two alternative nonrelevant features were added. It was not possible to provide two alternative nonrelevant features for one of the original sets of stimuli (influenza stimuli) while maintaining some relation to naturally occurring biological stimuli. Therefore, the influenza stimuli were replaced with the sensory neuron stimuli. The participants who had completed baseline with the original stimuli were invited to continue participating when the new stimuli were created (Participants 201, 202, 204, 205, and 208 continued), even if they had previously been dismissed for responding correctly during baseline (Participants 204 and 205).

\section{Procedure}

An example of four stimuli that could be presented in a trial and descriptions of the relation between these stimuli and each training condition are provided in Appendix M (Experiment 2 stimuli). Baseline, training, and test trials were conducted in a manner similar to Experiment 1 except for the differences outlined below.

Baseline Dismissal Criterion. Because the stimuli in Experiment 2 were based on real biological concepts, it was possible that the participants could already be familiar with these concepts. The first eight participants (201-208) were dismissed if, in any baseline block, a participant correctly selected examples of the biological concept in 18/24 trials. However, 
because any response during trials with training stimuli in the Examples-Only condition was necessarily correct, the criterion was modified such that participants were dismissed from the session only if they correctly selected examples of the biological concept during all (6/6) trials with untrained close-in nonexamples. The next session began with baseline blocks in the next condition. This process was repeated until baseline in all conditions was completed, at which point the participant was paid the money they earned and dismissed from the experiment.

Concept Description. After testing in each condition, participants wrote answers to two open-ended questions (see Appendix N). First, the participants were asked to name the concept they were taught. Next, the participants were asked to describe how they selected an image. This survey was not included in Experiment 1 because the same stimuli were used across conditions, so these questions may have prompted answers that then affected responding on future conditions.

\section{Results and Discussion}

As in Experiment 1, visual inspection was used to compare concept formation across training with examples, training with examples and far-out nonexamples, and training with examples and close-in nonexamples. In each condition, accuracy was compared across baseline and test trials (if they were completed). Recall that training and testing were not completed if correct stimuli were selected on all baseline trials with examples and close-in nonexamples (updated neuron, plant, or bacteria stimuli) or with examples and far-out nonexamples (original influenza stimuli). Graphs of data for the four participants who did not complete the experiment are shown in Appendix O.

The graphs in Figure 12 show accuracy in each of the six baseline and testing blocks in each condition for Participants 205 and 202, both of whom completed baseline and testing in all 
conditions at least once. As noted in the Method, Participant 205 was dismissed for responding accurately during baseline with the original stimuli (influenza) but resumed participation with the new stimuli (neuron). Conditions are shown in the order in which they were completed.

Responding on test trials with training stimuli (triangles in the top graphs) should remain accurate during testing, as was observed across all conditions for Participant 205 and 202 except the Far-Out Nonexamples conditions with neuron stimuli for 202. It is possible that responding was disrupted during testing, as discussed following Experiment 1. However, the pattern of disruption differs from Experiment 1 in that responding on trials with training stimuli only decreased during this Far-Out Nonexamples condition.

Across all conditions for Participants 205 and 202, accuracy was low on trials with untrained examples and close-in nonexamples (circles in the bottom graphs) during baseline, and only improved during testing following training in the Close-In Nonexamples condition. Accuracy on trials with examples and untrained far-out nonexamples (squares in the bottom graphs) only improved across baseline and testing for Participant 202 in the Far-Out Nonexamples condition with influenza stimuli. Accuracy on these trials remained low across baseline and testing for Participant 205 in the Examples-Only condition with neuron stimuli. In all other conditions across both participants, accuracy on trials with far-out nonexamples was moderate to high (albeit sometimes variable) during baseline, suggesting that participants discriminated between examples and nonexamples prior to training. 
Figure 12

Baseline and Testing Accuracy for Participants 202 and 205

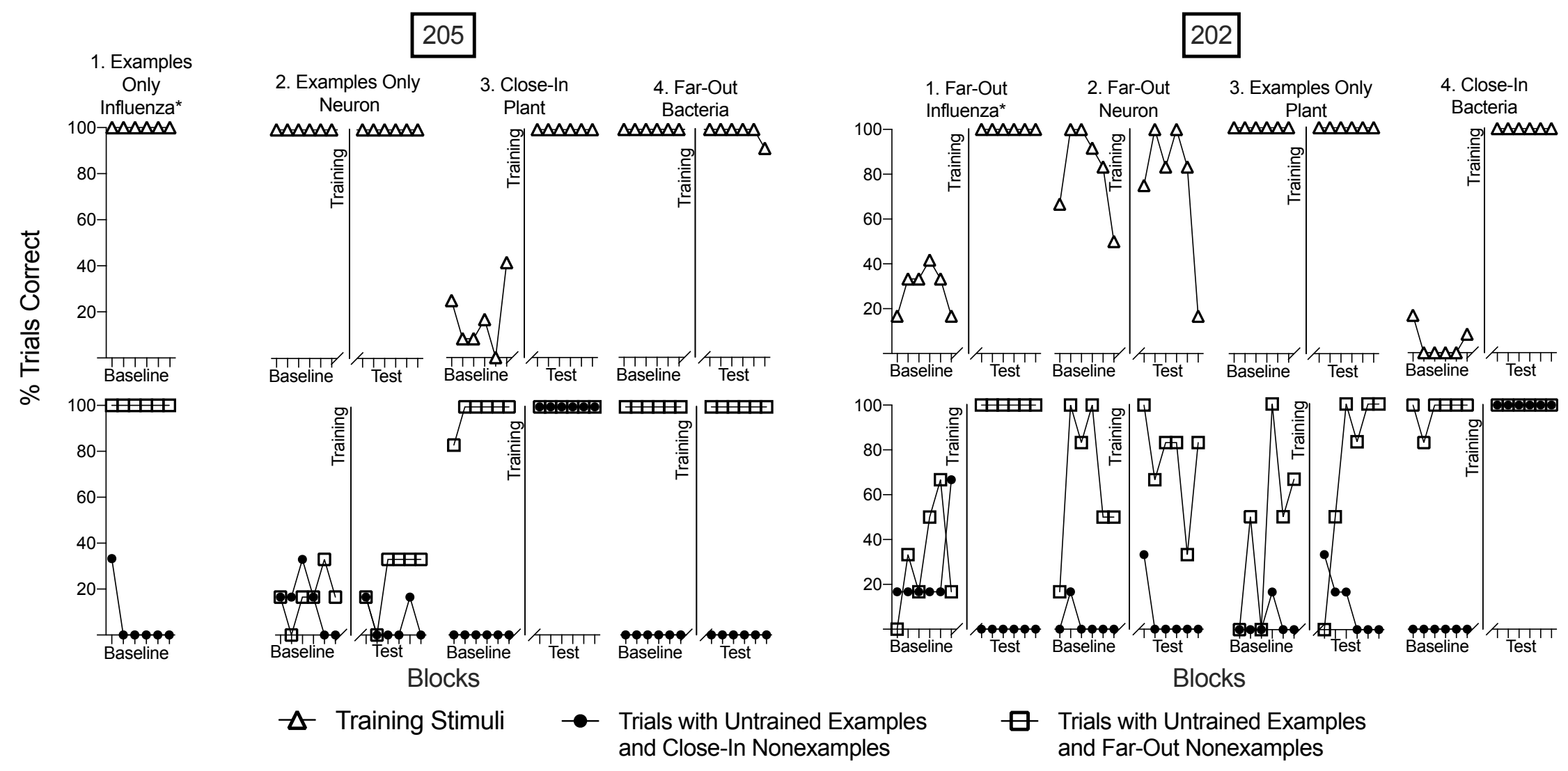

* Original set of experimental stimuli was used. Each relevant feature was replaced with only one replacement feature.

Note. Training blocks are indicated by the solid, vertical line between baseline and testing blocks, though data from these blocks are not displayed. The conditions are indicated by the names above the graph and appear in the order in which they occurred. 
There are at least two explanations for relatively accurate responding during baseline on trials with untrained far-out nonexamples. First, because the concepts were biological, participants may have had a history with them. However, if this were the case, participants might have been able to report the name of the concept or describe the features in biological terms. Neither Participant 205 nor Participant 202 used any biological term when asked to name and describe the concept (see Appendix P for each participant's responses), so knowledge of the concept, if any, was limited. Second, there may be relations among stimuli, both within and across trials, that enabled the participant to identify the correct stimulus. A participant may choose a stimulus based on a particular feature, and they may be more likely to select a feature that is reliably present in all trials. Each relevant feature was always present in exactly one stimulus in every trial, whereas irrelevant and replacement features could be present in zero to three (replacement features) or four (irrelevant features) stimuli in each trial. Selecting a response with a feature that is always present would result in accurate responding before training. Accurate baseline responding only occurred during Experiment 2, which may be due to differences in the creation of the arbitrary and biological stimuli (discussed in more detail in the General Discussion). Future research could create nonexamples and arrange trials such that each replacement feature was present as frequently as each relevant feature.

Graphs of data for Participant 211 are shown in Figure 13. Accuracy on testing trials with training stimuli improved relative to baseline in the Close-In Nonexamples condition but remained below 100\%. Following training in the Close-In Nonexamples condition, accuracy remained low on testing trials with untrained close-in nonexamples. Similar to Participants 103, 107, and 106 in Experiment 1, it is possible that a lack of motivation or disruption following the presentation of untrained stimuli may have resulted in the decreased accuracy on test trials with 
both training and untrained stimuli. Accuracy on testing trials with untrained close-in nonexamples also remained low across baseline and testing in the Examples-Only and Far-Out Nonexamples condition. Accuracy on test trials with untrained far-out nonexamples increased between baseline and testing in the Close-In Nonexample condition. Accuracy on test trials with untrained far-out nonexamples also improved gradually following training in the Examples-Only condition, even though no nonexamples were included during training. Similarly, accuracy on trials with far-out nonexamples (both training and untrained) was high during baseline in the FarOut Nonexamples condition indicating that the participant discriminated between examples and far-out nonexamples without training with far-out nonexamples.

Figure 13

Baseline and Testing Accuracy for Participant

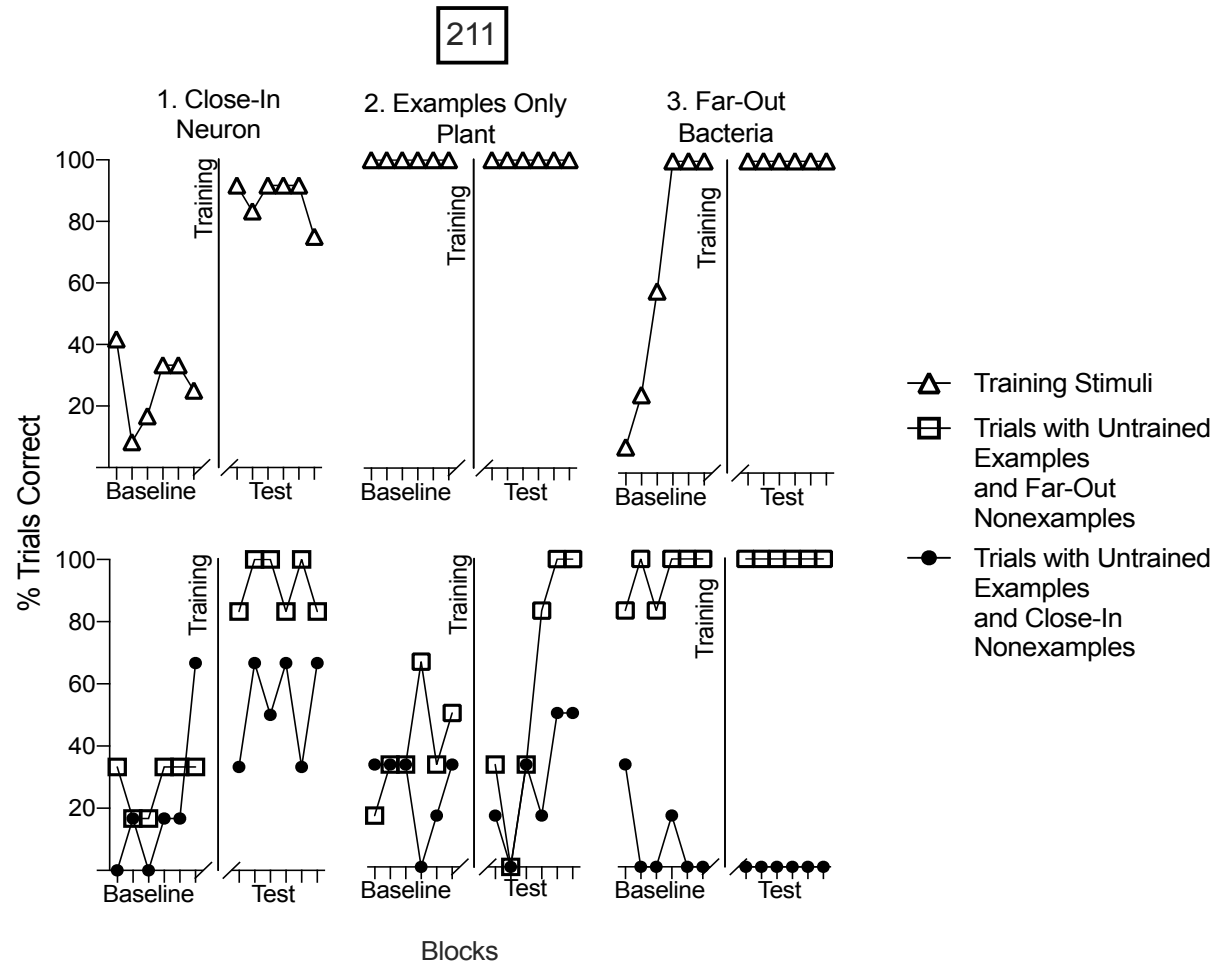

Note. Training blocks are indicated by the solid, vertical line between baseline and testing blocks, though data from these blocks are not displayed. The conditions are indicated by the names above the graph and appear in the order in which they occurred. 
Graphs of data for Participant 209 are shown in Figure 14. In the Close-In and Far-Out Nonexample conditions, accuracy increased relative to baseline on trials with training stimuli and untrained far-out nonexamples. As was the case for Participants 205 and 202, accuracy increased on trials with untrained examples and close-in nonexamples following training in the Close-In Nonexamples condition. Accuracy on trials with untrained examples and close-in nonexamples also gradually improved following training in the Far-Out Nonexamples condition, suggesting that discrimination between close-in nonexamples and examples was learned over the course of the testing blocks, even though no feedback on accuracy was provided. Recall that, on trials with far-out nonexamples, examples could be identified by selecting the stimulus with one of the three relevant features because these features were always present in exactly one stimulus. That exact strategy would not work to select examples on trials with close-in nonexamples because each nonexample lacks only one relevant feature, so more than one stimulus could have each relevant feature. However, rather than learn to discriminate between the presence and absence of a single relevant

Figure 14

\section{Baseline and Testing Accuracy for Participant 209}

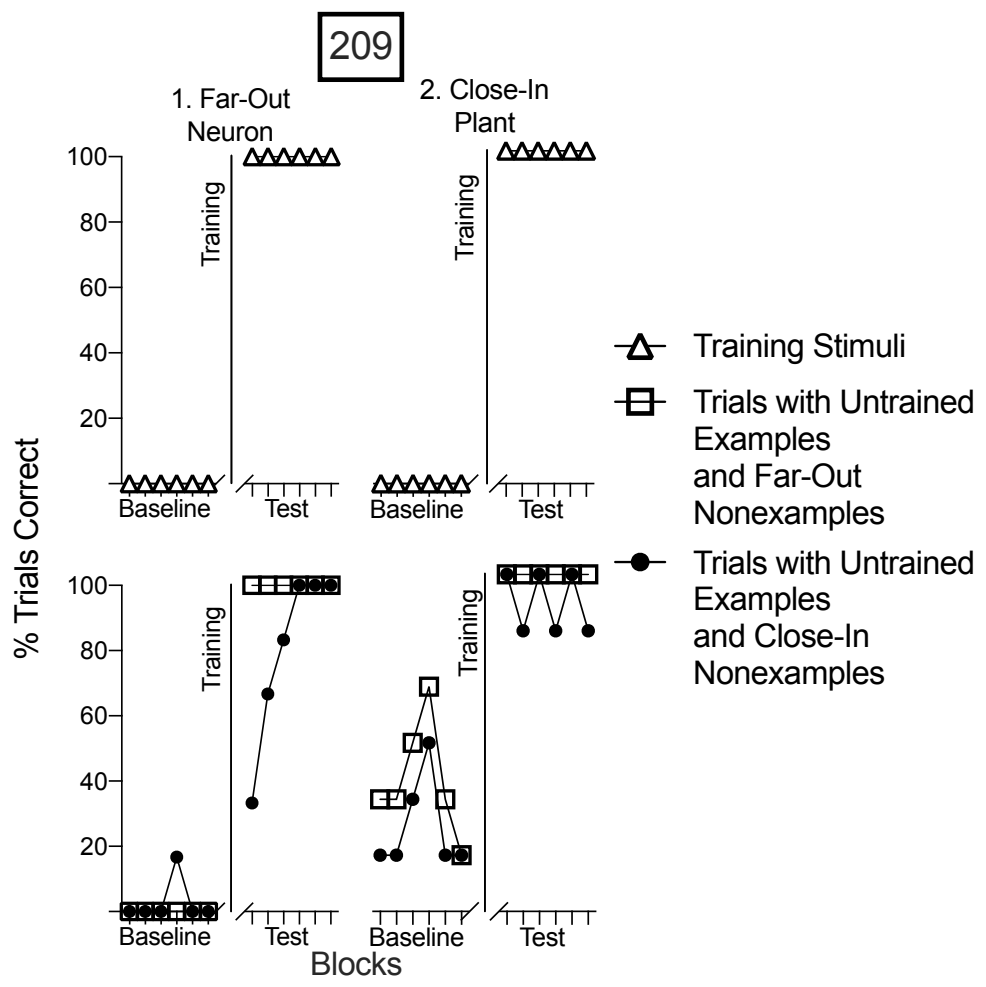

Note. Training blocks are indicated by the solid, vertical line between baseline and testing blocks, though data from these blocks are not displayed. The conditions are indicated by the names above the graph and appear in the order in which they occurred. The participant withdrew prior to completing the third (Examples Only) condition. 
feature, they could learn to discriminate the presence and absence of a particular combination of features. In trials with close-in nonexamples, all three relevant features were always present on exactly one stimulus (the example). The other three stimuli (close-in nonexamples) each had a random combination of one replacement feature and two relevant features (12 possible combinations). The example could be selected by identifying the combination of three features that occurred most frequently and was always present in each trial. The strategies used to identify examples in conditions with close-in and far-out nonexamples could be combatted by modifying the number of stimuli presented in each trial or increasing the number of irrelevant features (thus making it harder to identify relevant features). In the case of Participant 209, even though responses became more accurate during testing with untrained close-in nonexamples after training in the Far-Out Nonexamples condition, accuracy was low at baseline for the next condition, suggesting the method for selecting untrained examples and close-in nonexamples did not generalize to another stimulus set prior to training.

Graphs of data for Participants 201, 210, 204, and 208 are shown in Figure 15. Whenever training occurred, responding on test trials with training stimuli improved relative to baseline. All of these participants responded accurately during baseline trials with untrained examples and nonexamples after completing training in at least one other condition. Both participants who completed the Close-In Nonexamples condition first (201 and 210) responded accurately on all trials by the end of baseline in all subsequent conditions, regardless of stimulus set. Both participants who completed Examples Only first (204 and 208) responded accurately on all trials at baseline after completing training in multiple conditions. Across all four participants, baseline accuracy increased on trials with untrained far-out nonexamples before it increased on trials on untrained close-in nonexamples. Training only took place if accuracy never reached $100 \%$ on 
baseline trials with untrained examples and close-in nonexamples. When training did occur, accuracy on trials with untrained examples and close-in nonexamples only increased between baseline and testing in the Close-In Nonexamples condition. Following completion of the study, each participant was asked to describe how they chose the correct stimulus (see Appendix Q for their responses). All four participants described looking for features that occurred together on every trial, suggesting that their correct responding at baseline can be attributed to the relations among stimuli across trials, not to previous knowledge of the biological concepts. In addition, accuracy on baseline trials with untrained examples and close-in nonexamples occurred at least once for each set of stimuli (neurons, plants, and bacteria), suggesting that accurate baseline responding cannot be attributed to features of a particular stimulus set.

Training procedures that are likely to produce generative repertoires could be applied to classroom settings to facilitate "learning to learn" (Harlow, 1949). It is not clear exactly what conditions may be most likely to support the development of a strategy to identify examples prior to training. However, all participants who responded accurately on baseline trials with untaught close-in nonexamples completed at least one condition with nonexamples, suggesting that nonexamples are necessary, though not always sufficient, to produce these generative repertoires. Each stimulus set in this experiment was created with similar arrangements of relevant, irrelevant, and replacement features so that the concept assigned to each condition was unlikely to affect concept formation. Arranging the stimulus features similarly across concepts may have facilitated the development of generative repertoires. Future research could evaluate ways of arranging related concepts in classroom settings to facilitate learning across concepts. 
Figure 15

Baseline and Testing Accuracy for Participants 201, 210, 204, and 208
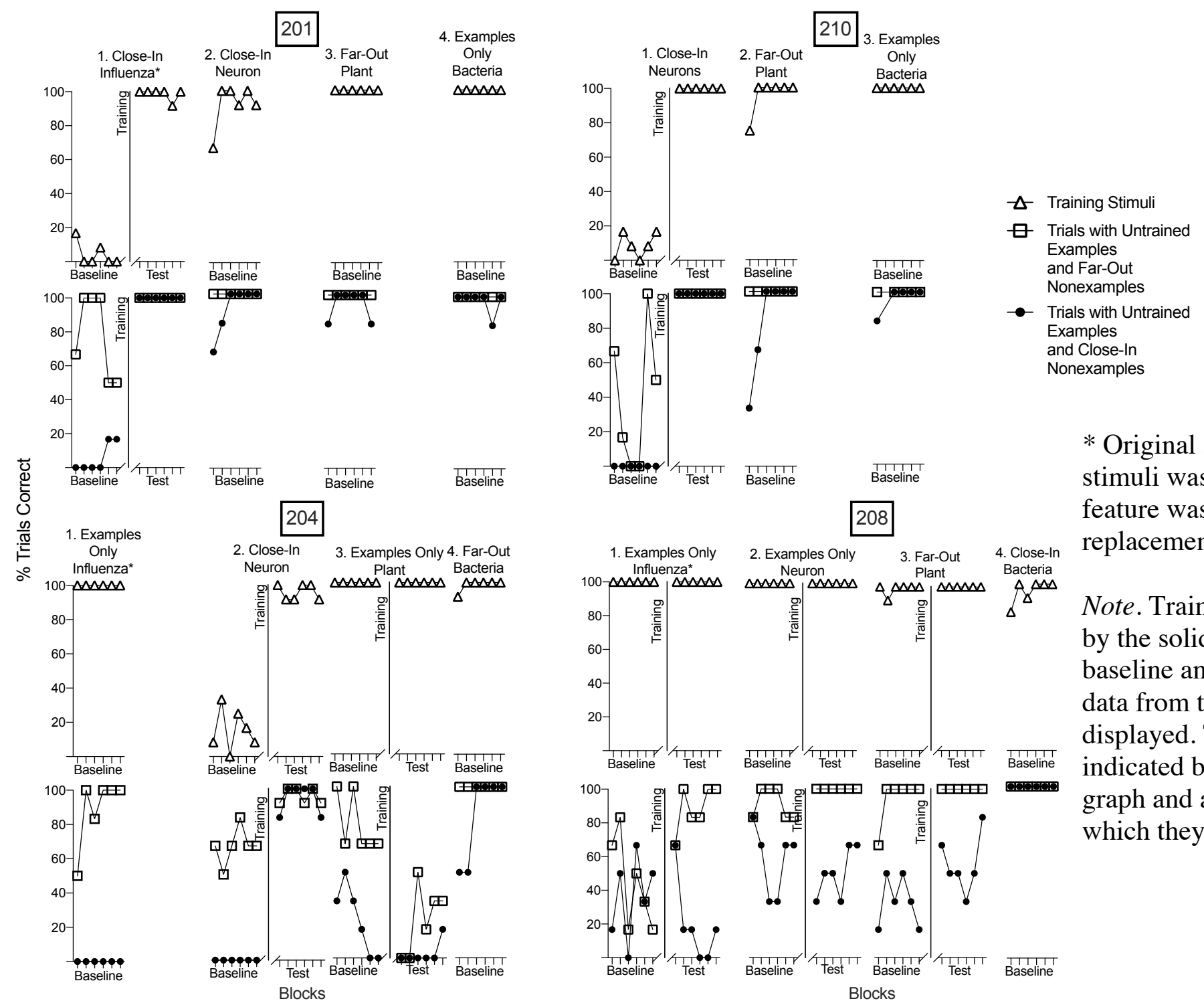

* Original set of experimental stimuli was used. Each relevant feature was replaced with only one replacement feature.

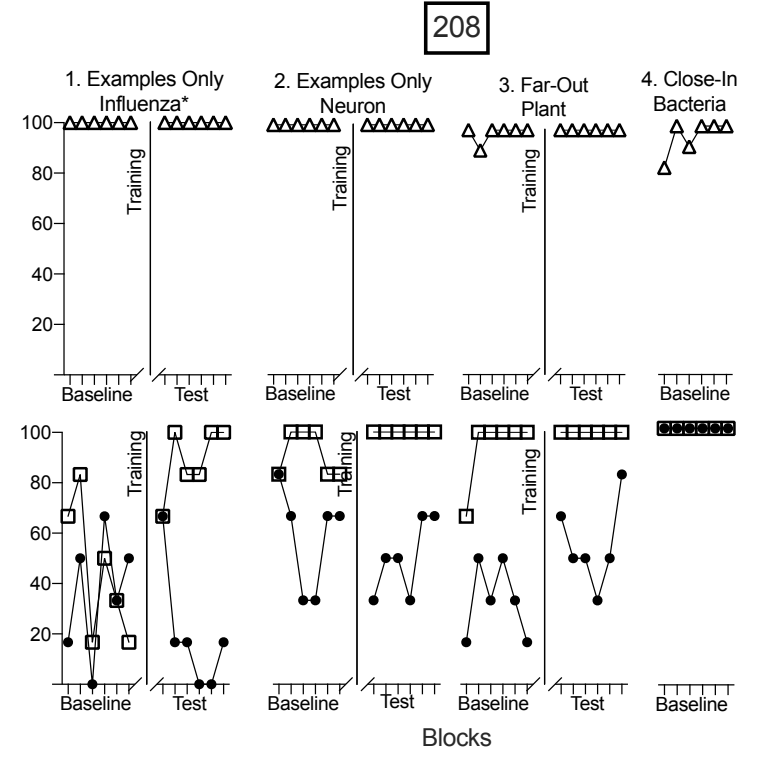

Note. Training blocks are indicated by the solid, vertical line between baseline and testing blocks, though data from these blocks are not displayed. The conditions are indicated by the names above the graph and appear in the order in which they occurred. 
Relative to baseline, accuracy increased on test trials with untrained examples and closein nonexamples across all participants who had completed training in the Close-In Nonexamples condition, but accuracy generally did not increase relative to baseline for participants who had completed training in the Far-Out Nonexamples condition. Even though training in the Close-In Nonexamples condition improved concept formation, it is also necessary to consider the time and resources needed to complete training with close-in nonexamples compared to far-out nonexamples. Figure 16 shows the number of blocks (left) and the number of correction trials (middle) completed during training in the Far-Out and Close-In Nonexamples conditions. Data from the Examples-Only condition were not included because all responses were correct; training always ended after the minimum number of blocks (4) and no correction trials. No

Figure 16

Number of Additional Training Blocks, Correction Trials, and Time to Complete Training in each Condition

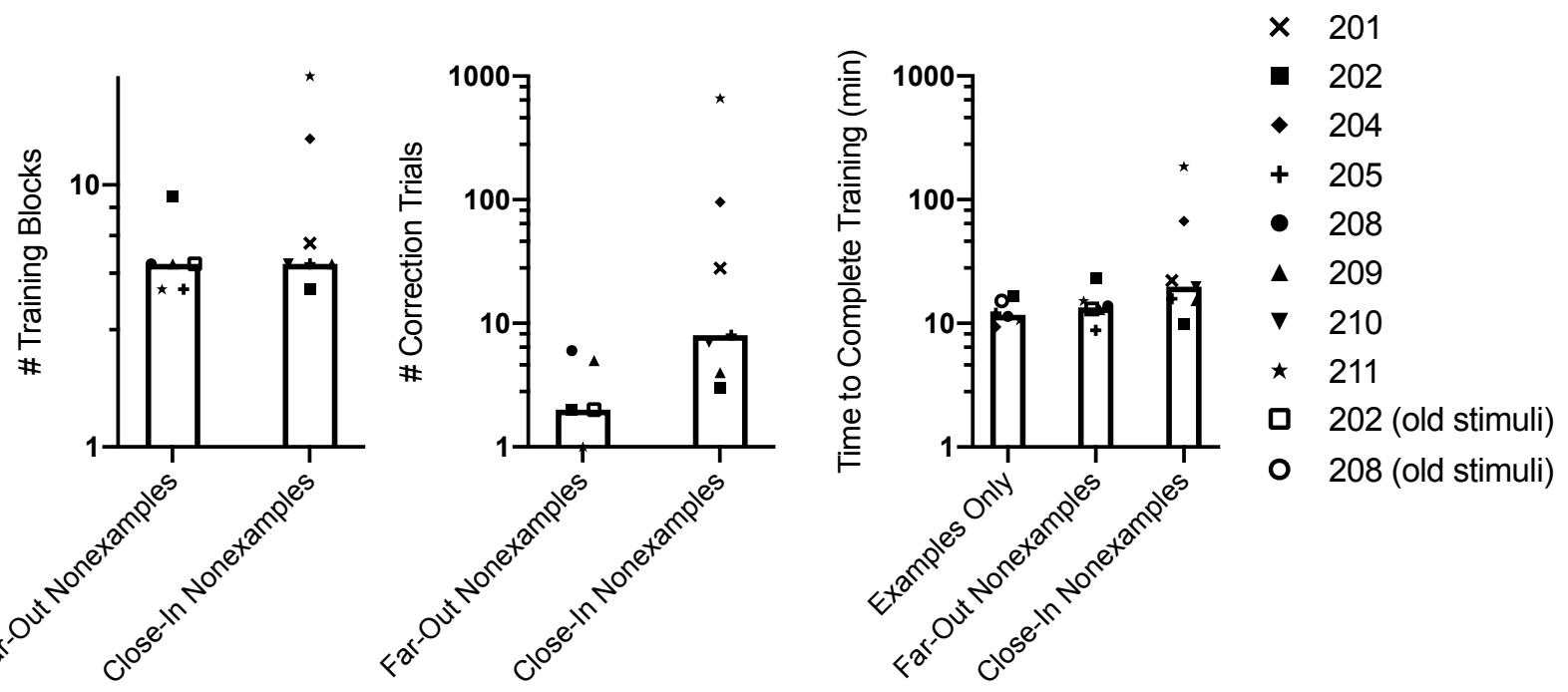

Note. The height of the bar depicts the median and each symbol depicts an individual participant. The $y$-axis has been scaled logarithmically to account for the large range in values. Participant 205 did not complete any correction trials in the Far-Out Nonexamples condition, so no data point was shown. 
statistical analysis was conducted to compare the number of training blocks or correction trials across conditions because the number of participants who completed training in both the CloseIn and Far-Out Nonexample conditions (Participants 201, 204, 208, 210) was insufficient to conduct a Wilcoxon signed-rank test. Based on visual inspection, the number of training blocks completed in the Far-Out and Close-In Nonexamples conditions was similar, but more correction trials were typically required to complete training in the Close-In Nonexamples condition than in the Far-Out Nonexamples condition.

The right graph in Figure 16 shows the total duration of training in each of the three conditions (including the Examples-Only condition). When compared using a Kruskal-Wallis test, the time to complete training in the Examples-Only ( $M d n=11.74 \mathrm{~min})$, Far-Out Nonexamples $(M d n=13.45 \mathrm{~min})$, and Close-In Nonexamples $(M d n=19.74 \mathrm{~min})$ conditions did not differ statistically significantly $(H(2)=5.21, \mathrm{p}=0.071)$. In sum, training in the Close-In Nonexamples condition resulted in more incorrect responses during training than training with far-out nonexamples but did not require significantly more blocks or time to complete. Therefore, training with far-out nonexamples and close-in nonexamples required similar resources, but only close-in nonexamples resulted in correct responding on test trials with untrained close-in nonexamples. At least in the context of the concepts taught in this experiment, it may be better to train with close-in nonexamples even if time is limited.

As in Experiment 1, potential emotional effects were evaluated for each training condition by having the participants complete the PANAS-X after the third consecutive training block in each condition with correct responses on at least 23 out of 24 trials. Figure 17 displays the median positive (left graph) and negative (right graph) affect score in each condition, where a higher score indicates more positive and negative affect. The median positive-affect score in the 
Examples-Only, Far-Out Nonexamples, and Close-In Nonexamples condition was 14.5, 16, and 20, respectively. The median negative-affect score in the Examples-Only, Far-Out Nonexamples, and Close-In Nonexamples condition was 13.5, 14.5, and 12, respectively. When compared using a Kruskal-Wallis test, as in Experiment 1, there were no statistically significant differences in positive $(H(2)=5.611, \mathrm{p}=0.055)$ or negative $(H(2)=0.061, \mathrm{p}=0.73)$ affect. A measure of affect was included because it was hypothesized that frequent incorrect responses could result in more negative emotional responding. Even though the most incorrect responses tended to occur in the Close-In Nonexamples condition, positive affect was highest and negative affect was lowest in this condition. These findings provide additional support that Close-In Nonexamples can be used during training without systematically worsening affect, even if more incorrect responses were made in this condition.

Figure 17

Note. The height of the bar depicts the median, and each symbol depicts an individual participant. A higher score indicates more positive or negative affect, and the maximum score is 50 .
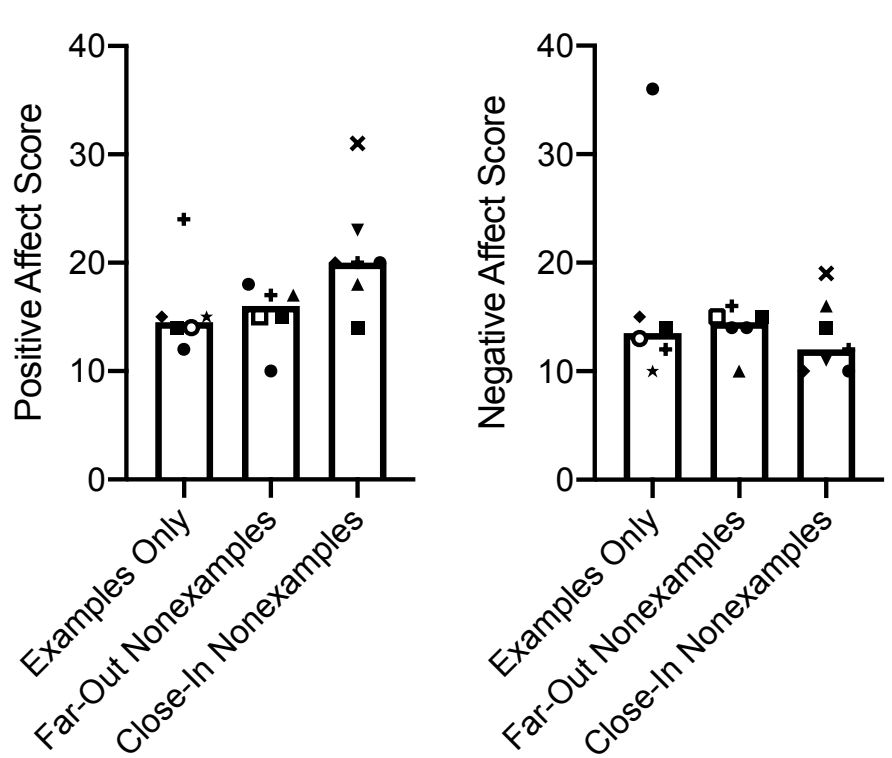

$\times \quad 201$

- 202

- 204

$+205$

- 208

- 209

จ 210

$\star 211$

ㅁ 202 (old stimuli)

o 208 (old stimuli)

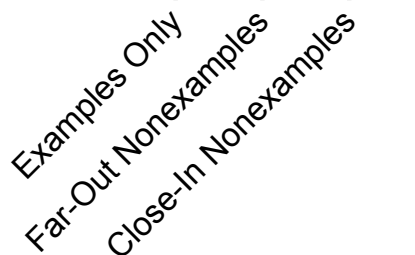

General Discussion

The present experiment compared concept formation following training with only examples, examples and far-out nonexamples, and examples and close-in nonexamples. Across both Experiment 1 (arbitrary stimuli) and Experiment 2 (biological stimuli), when only examples 
were presented during training, concept formation (as measured by increases in accuracy on trials with untrained close-in nonexamples across baseline and testing) never occurred (see Appendix R for a summary of outcomes across both experiments). As in previous research, presenting nonexamples during training was necessary for concept formation (e.g., Shumway \& Lester, 1974). In addition to the presence of nonexamples, the relation between examples and nonexamples also affected conceptual learning. Concept formation only occurred during testing after training in the Close-In Nonexamples condition. These results support the use of close-in nonexamples during training when discrimination between examples and close-in nonexamples is a desired outcome.

Although training in the Close-In Nonexamples condition facilitated concept formation, it was not always sufficient. Of the 13 participants who completed training in the Close-In Nonexamples condition, concept formation was observed for eight. Of the five participants for whom concept formation was not observed, accuracy on test trials with training stimuli decreased relative to training trials for all but two participants (108, who initially did not respond accurately during training in the Close-In Nonexamples condition, and 211). Accuracy may have decreased between training and testing because participants were not paid for correct responses during testing or because responding was disrupted by the introduction of untrained stimuli. Decreased accuracy on trials with training stimuli complicates the interpretation of inaccurate responses on trials with untrained stimuli. To promote accuracy on testing trials with training stimuli, future research could continue to pay participants for correct responses during testing or expand training to include trials with neutral feedback (as described in more detail in the Experiment 1 Results and Discussion). 
Across both experiments there was some evidence that the condition order might affect concept formation, even though a different concept was taught in each condition. In Experiment 1, Participant 108 completed the Close-In Nonexamples condition first and never responded accurately during training. When this condition was repeated after the Examples-Only and FarOut Nonexamples conditions, accurate responding occurred during training, suggesting that completing these conditions improved accuracy in the Close-In Nonexamples condition. In Experiment 2, four participants responded accurately on trials with untrained close-in nonexamples before completing training. All of these participants had already completed training in either the Close-In (Participants 201, 210, and 204) or Far-Out (Participant 208) Nonexamples condition, suggesting that the Examples-Only condition may not be sufficient to produce strategies to identify examples prior to training. Both participants in Experiment 1 (Participant 107 and 108) and one (Participant 211) of three participants in Experiment 2 who completed the Close-In Nonexamples condition first did not respond accurately on test trials, suggesting that concept formation may not improve as much following only training in the Close-In Nonexamples conditions when previous training conditions had not also been completed, even though different concepts were taught in each condition. Because a different concept was taught in each condition and the order of conditions was counterbalanced across participants in both experiments, it is unclear exactly how the condition order affects concept formation. It is possible that training the same concept using far-out nonexamples then close-in nonexamples (or both simultaneously) may improve concept formation relative to training using just close-in or far-out nonexamples. Future research should manipulate the order of different combinations of example and nonexamples to compare concept formation across different training sequences. 
Generally, participants in Experiment 2 responded more accurately than participants in Experiment 1 in at least three ways. First, accuracy for more participants in Experiment 2 improved on test trials with untrained examples and close-in nonexamples than participants in Experiment 1. Second, four of seven participants in Experiment 2 responded accurately on baseline trials with untrained close-in nonexamples, whereas this did not happen for any participants in Experiment 1. Third, relative to training in the Far-Out Nonexamples condition, training in the Close-In Nonexamples condition required more blocks, correction trials, and time in Experiment 1, but only more correction trials in Experiment 2. All three of these differences indicate that the Experiment 2 concepts were easier to learn than the Experiment 1 concepts.

Recall that arbitrary stimuli were used in Experiment 1 to minimize effects of previous learning histories whereas biological stimuli were used in Experiment 2 to mimic the kinds of concepts that would be taught in a classroom. It is possible that the differences between experiments were the result of participants' histories with the concepts in Experiment 2. However, there were at least three differences in the relations between relevant, irrelevant, and replacement features in Experiment 1 compared to Experiment 2 that could also account for the differences observed across experiments.

First, the arrangement of features across the Experiment 2 stimuli (as discussed in more detail in the Experiment 2 Results and Discussion) may have allowed participants to identify the example in the absence of feedback because the three relevant features were present together more often than any combination of irrelevant features. In Experiment 1, participants could not identify examples in this way because multiple combinations of irrelevant features were presented together as often as the three relevant features. When it is necessary to establish experimental control by preventing concept formation in the absence of feedback, researchers 
should include stimuli across trials such that any combination of irrelevant features is as likely to occur as the relevant features. On the other hand, to facilitate concept formation in classroom settings, it may be beneficial to present the relevant features more frequently than any particular combination of irrelevant features. For example, if teaching the concept "square", one option should be a square and the other option should vary across trials.

Second, the irrelevant features for each concept in Experiment 2 (color, orientation, and location of each feature) were the same, but the irrelevant features for each concept in Experiment 1 differed across concepts. Thus, in only Experiment 2, generalization across examples with irrelevant features during one condition could be applied to examples in other conditions. If it is not desirable for concept formation for one concept to facilitate concept formation for another (i.e., concepts taught need to be independent), different irrelevant features should be used for each concept. If it is desirable for concept formation for one concept to facilitate concept formation for another (e.g., teaching related concepts in a classroom), then it may be beneficial to use parallel irrelevant features when possible.

Third, each irrelevant feature was present an equal number of times in all conditions in Experiment 2, but some irrelevant features were present more than others across conditions in Experiment 1 so that the same set of stimuli could be used across conditions. Song et al. (2021) compared training and testing in two conditions: one in which each irrelevant feature was presented equally and one in which one irrelevant feature was presented more often (78\% of trials) than the other two (11\% each). They found that responding on trials with untrained stimuli was similar following training in both conditions, but training required fewer trials when the irrelevant features were presented equally. In the present Experiment 1, irrelevant features were presented equally in the Close-In Nonexamples condition but unequally in the Far-Out 
Nonexamples condition. Based on the findings of Song et al., the unequal distribution of irrelevant features should slow acquisition in the Far-Out Nonexamples condition relative to the Close-In Nonexamples condition, but only in Experiment 1. However, the opposite was observed, as training in the Far-Out Nonexamples condition was consistently faster than training in the Close-In Nonexamples condition in Experiment 1, but not in Experiment 2. These results suggest that how often each irrelevant feature is present may be less important than the number of relevant features missing from nonexamples. Future research could investigate the interaction between the number of missing relevant features and relative prevalence of each irrelevant feature.

Despite the aforementioned differences, both experiments share some limitations. Previous research has demonstrated that training using examples and nonexamples with different irrelevant features (i.e., unmatched examples and nonexamples) is less likely to result in concept formation than training using examples and nonexamples with identical irrelevant features (i.e., matched examples and nonexamples; Merrill \& Tennyson, 1978; Tennyson et al., 1972;

Tennyson, 1973). Although there were matched examples and nonexamples in each stimulus set used in this experiment, the exact examples and nonexamples displayed in each trial were randomized. Therefore, the number of training trials with a matched example and nonexample pair may differ across conditions, although these differences should not be systematic. Training with matched examples and nonexamples could improve concept formation above and beyond training with close-in nonexamples. Future research could investigate the interaction between matched examples and nonexamples and the use of different types of nonexamples.

Another general limitation of the present experiments is that a fairly small number of examples (six) were used in the Close-In and Far-Out training conditions. It is possible that 
participants could memorize each example (discriminate between the presence and absences of all features, even if they were not relevant) rather than discriminate between the presence and absence of only the relevant features. When asked to name the concept they learned, three participants in Experiment $2(202,204$, and 211) indicated that they may have memorized the examples of the concept in the Close-In Nonexamples condition. Accuracy during training could occur if the six examples were memorized but would not result in accurate responding on test trials with untrained stimuli. Accuracy increased between baseline and testing on trials with untrained stimuli for both Participants 202 and 204, indicating that they learned the concept even though they called this concept "memorization". For 211, accuracy increased between baseline and testing only on trials with untrained examples and far-out nonexamples, even though responding on trials with training stimuli remained above baseline level, which may indicate she did memorize the training stimuli. A larger set of training stimuli could be used to mitigate memorization (e.g., Kodak et al., 2020; Nakamura et al., 2008).

The concepts taught in Experiment 2 were selected to represent concepts that could be taught in the classroom, but they were intentionally taught in the absence of typical classroom instruction (i.e., presentation of rules or definitions) to isolate the effects of nonexamples during instruction. Previous investigations on matched examples and nonexamples conducted in the classroom (Merrill \& Tennyson, 1978; Tennyson et al., 1972; Tennyson, 1973) provided the students with a definition of the concept prior to training. The inclusion of a definition alone can improve concept formation (e.g., Klausmeier et al., 1973). Definitions may teach the relevant and irrelevant features of a concept, moderating the effects of examples and nonexamples on concept formation. Future research should evaluate effects of nonexamples on concept formation in classroom settings to identify best practices for teaching concepts. However, research should 
also continue to evaluate concept formation in the absence of verbal instructions to isolate the effects of nonexamples and produce findings that more likely to generalize to populations that cannot follow instructions or understand definitions.

The results of the present experiments, in combination, demonstrate how the stimuli used to train concepts affect concept formation. Training in the Close-In Nonexamples condition resulted in better concept formation than training in the Far-Out Nonexamples and ExamplesOnly conditions, indicating that the relation between examples and nonexamples affects concept formation. When the Experiment 2 stimuli were modified to include two replacement features, fewer participants responded accurately during baseline at the start of the experiment compared to when the stimuli included only one replacement feature, providing evidence that the number of replacement features may affect concept formation. The relative findings between Experiments 1 and 2 suggest that the number of irrelevant features and relative probability of the different feature combinations also affect concept formation. Future research should manipulate these variables to provide recommendations for best practice when selecting stimuli to teach concepts.

Despite the numerous suggested effects of stimulus features on concept formation, few experiments have been conducted to demonstrate these effects. In addition, although stimulus sets are often used in evaluations of different instructional techniques, few provide descriptions of the stimuli that are detailed enough to allow for the relation among stimulus features to be evaluated (e.g., Schnell et al., 2018) making it difficult to consider the relation among stimulus features when interpreting disparate findings across studies. In general, research on concept formation will benefit if researchers provide descriptions of stimuli or provide the stimulus sets as supplemental material to allow for these evaluations. Overall, the present experiments provide 
evidence that relations between examples and nonexamples, both within and across trials, affect concept formation and supporting the use of close-in nonexamples to promote concept formation. 


\section{References}

Binder, C. (1996). Behavioral fluency: Evolution of a new paradigm. The Behavior Analyst, 19(2), 163-197. https://doi.org/10.1007/BF03393163

Bodily, K. D., Katz, J. S., \& Wright, A. A. (2008). Matching-to-sample abstract-concept learning by pigeons. Journal of Experimental Psychology: Animal Behavior Processes, 34(1), 178-184. https://doi.org/10.1037/0097-7403.34.1.178

Broschard, M. B., Kim, J., Love, B. C., Wasserman, E. A., \& Freeman, J. H. (2019). Selective attention in rat visual category learning. Learning \& Memory, 26(3), 84-92. http://doi.org/10.1101/1m.048942.118

Cedro, Á. M., Borges, J., Diniz, M. L. N., Rodrigues, R. M., Rico, V. V., Leme, A. C., \& Huziwara, E. M. (2019). Evaluating concept formation in multiple exemplar training with musical chords. The Psychological Record, 69(3), 379-391. http://doi.org/10.1007/s40732-019-00346-5

Fleming, M. L., \& Levie, W. H. (1993). Instructional message design: Principles from the behavioral and cognitive sciences. Educational Technology.

Galizio, M., Stewart, K.L. and Pilgrim, C. (2004). Typicality effects in contingency-shaped generalized equivalence classes. Journal of the Experimental Analysis of Behavior, 82: 253-273. https://doi.org/10.1901/jeab.2004.82-253

Harlow, H. F. (1949). The formation of learning sets. Psychological Review, 56(1), 5165. https://doi.org/10.1037/h0062474

Klausmeier, H. J. \& Feldman, K. V. (1973). The effects of a definition and a varying number of examples and nonexamples on concept attainment (250). Wisconsin research and 
development center for cognitive learning. Retrieved July 21, 2020, from https://files.eric.ed.gov/fulltext/ED085684.pdf

Khallad, Y. (2004). Conceptualization in the pigeon: What do we know?. International Journal of Psychology, 39(2), 73-94. https://doi.org/10.1080/00207590344000349

Kodak, T., Halbur, M., Bergmann, S., Costello, D. R., Benitez, B., Olsen, M., ... \& Cliett, T. (2020). A comparison of stimulus set size on tact training for children with autism spectrum disorder. Journal of applied behavior analysis, 53(1), 265-283. https://doi.org/10.1002/jaba.553

Lazarowski, L., Goodman, A., Galizio, M., \& Bruce, K. (2019). Effects of set size on identity and oddity abstract-concept learning in rats. Animal Cognition, 22(5), 733-742. http://doi.org/10.1007/s10071-019-01270-5

Markle, S. M., \& Tiemann, P. W. (1970a). Problems of conceptual learning. Journal of Educational Technology, 1(1), 52-62. Retrieved July 21, 2020, from https://eric.ed.gov/?id=EJ024688

Markle, S. M., \& Tiemann, P. W. (1970b). "Behavioral” analysis of "cognitive" content. Educational Technology, 10(1), 41-45. Retrieved July 21, 2020, from http://www.jstor.org/stable/44423095

Markle, S. M. (1990). Designs for instructional designers (3 ${ }^{\text {rd }}$ ed.). Morningside Press. Mechner, F. (1965). Science education and behaviour technology. In R. Glaser (Ed.), Teaching Machines and Programmed Learning II (pp. 441-507) National Education Association.

Merrill, M. D., \& Tennyson, R. D. (1978). Concept Classification and Classification Errors as a Function of Relationships between Examples and Nonexamples. Improving Human Performance Quarterly, 7(4), 351-64. 
Merrill, M. D., Tennyson, R. D., Posey, L. O. (1992). Teaching Concepts (2 ${ }^{\text {nd }}$ ed.). Education Technology Publications.

Nakamura, T., Wright, A. A., Katz, J. S., Bodily, K. D., \& Sturz, B. R. (2009). Concept learning carryover effects from the initial training set in pigeons (Columba livia). Journal of Comparative Psychology, 123(1), 79. http://doi.org/10.1037/a0013126

Seger, C. A., Braunlich, K., Wehe, H. S., \& Liu, Z. (2015). Generalization in category learning: the roles of representational and decisional uncertainty. Journal of Neuroscience, 35(23), 8802-8812. https://doi.org/10.1523/JNEUROSCI.0654-15.2015

Schnell, L. K., Vladescu, J. C., Kodak, T., \& Nottingham, C. L. (2018). Comparing procedures on the acquisition and generalization of tacts for children with autism spectrum disorder. Journal of Applied Behavior Analysis, 51(4), 769-783. https://doi.org/10.1002/jaba.480

Shumway, R. J., \& Lester, F. K. (1974). Negative instances and the acquisition of the mathematical concepts of commutativity and associativity. Educational Studies in Mathematics, 5(3), 301-315. https://doi.org/10.1007/BF01424552

Song, C. J., Vladescu, J. C., Reeve, K. F., Miguel, C. F., \& Breeman, S. L. (2020). The influence of correlations between nonrelevant features and reinforcement on stimulus generalization. Journal of Applied Behavior Analysis. https://doi.org/10.1002/jaba.760

Stokes, T. F., \& Baer, D. M. (1977). An implicit technology of generalization. Journal of Applied Behavior Analysis, 10(2), 349-367. https://doi.org/10.1901/jaba.1977.10-349

Tennyson, R. D. (1973). Effects of negative instances in concept acquisition using a verbal learning task. Journal of Educational Psychology, 64(2), 247-260. https://doi.org/10.1037/h0034400 
Tennyson, R. D., Woolley, F. R., \& Merrill, M. D. (1972). Exemplar and nonexampler variables which produce correct concept classification behavior and specified classification errors. Journal of Educational Psychology, 63(2), 144-152. https://doi.org/10.1037/h0032368

Tiemann, P., \& Markle, S. (1985). Analyzing instructional content: A guide to instruction and evaluation. Champaign. Stipes Publishing Co.

Wasserman, E. A. (2016). Conceptualization in pigeons: The evolution of a paradigm. Behavioural processes, 123, 4-14. http://doi.org/10.1016/j.beproc.2015.09.010

Watson, D., \& Clark, L. A. (1994). The PANAS-X: Manual for the Positive and Negative Affect Schedule-Expanded Form. Iowa City: Unpublished manuscript. University of Iowa.

Weeks, M., \& Gaylord-Ross, R. (1981). Task difficulty and aberrant behavior in severely handicapped students. Journal of Applied Behavior Analysis, 14(4), 449-463. https://doi.org/10.1901/jaba.1981.14-449

Woolley, F. R., \& Tennyson, R. D. (1972). Conceptual model of classification behavior. Educational Technology, 12(4), 37-39. https://www.jstor.org/stable/44417827

Zarcone, J. R., Iwata, B. A., Smith, R. G., Mazaleski, J. L., \& Lerman, D. C. (1994). Reemergence and extinction of self-injurious escape behavior during stimulus (instructional) fading. Journal of Applied Behavior Analysis, 27(2), 307-316. https://doi.org/10.1901/jaba.1994.27-307

Zentall, T. R., Galizio, M., \& Critchfield, T. S. (2002). Categorization, concept learning, and behavior analysis: An introduction. Journal of the Experimental Analysis of Behavior, 78(3), 237-248. https://doi.org/10.1901/jeab.2002.78-2 


\title{
Appendix A: Consent Form
}

\author{
Principal Investigator (PI) | Claire St. Peter, Ph.D., BCBA-D \\ Department | Psychology \\ WVU IRB Protocol \# | 2006046831
}

Study Title | Studying variables that affect learning using a computer task

\section{Why is this research being done and what is involved?}

The purpose of this study is to identify variables that affect learning. The impacts of information presentation and delivery of feedback on individuals' learning and use of information is currently unclear. To see how the way information is presented affects learning, you can earn rewards by figuring out how to respond correctly on arbitrary tasks created to mimic learning in the real world.

The study involves engaging in simple responses on a computer to earn or lose money. You may be paid this money at the end of the appointment or this money may be hypothetical. If you are a psychology student, you may receive course credit. The investigator will tell you which is the case for the current study. Your earnings will be displayed on the screen. It is up to you to figure out how to earn money.

Your participation will take between 1 and 20 hours. The exact amount of time required will vary depending on your responding and the experimental condition to which you are assigned. We will provide you with at least a 5-minute break after each hour that you participate. If your participation will last more than three hours, you will be required to come in for multiple appointments. Up to 26 appointments may be required to complete the study.

\section{Do I have to participate and what are the risks?}

Your participation in this research study is completely voluntary and you are free to withdraw from the research at any time. You may not receive any direct benefits from the study. The knowledge gained from this study may eventually benefit others.

There are no known or expected risks from participating in this study, except for the mild frustration associated with determining how to best earn money.

\section{Will I be compensated for my participation?}

You may earn extra credit for your Psychology courses or money for your participation. You cannot receive both course credit and monetary payment. If you earn extra credit, that credit will be awarded according to the policy on your instructor's syllabus. You will earn extra credit based on the amount of time that you spend participating. It may be possible for you to earn more extra credit than can be awarded in your class. You will not lose any extra credit that you have already earned if you withdraw from the study before its conclusion. Please consult with your instructor or read your syllabus for more details. Other options are available for earning the same credit. If you receive monetary compensation, you will be asked to provide your name, address, Social Security Number and verification of U.S Citizenship or Permanent Resident Status to receive payment. Your information, if obtained, may be provided to the appropriate parties for billing and/or payment purposes. Please be advised that any compensation received for participation in a research study, including a gift card, is considered taxable income and must be reported to the Internal Revenue Service (IRS). You will be paid based on your point earnings, which will range 
from $\$ 7$ to $\$ 20$ per hour. If you withdraw before the end of the study, no additional payments will be made but you will still receive the money you have earned so far.

\title{
What will happen to my research information and data?
}

Your data, research results, or any and all other information related to this research study used in this research study may contribute to a new discovery or treatment. In some instances, your data, your research results, these discoveries, or any other information related to this research study, even if identifiers are removed, may be of commercial value and may be sold, patented, or licensed by the investigators and West Virginia University for use in other research or the development of new products. You will not retain any property rights, nor will you share in any money or commercial profit that the investigators, West Virginia University, or their agents may realize.

Any information about you that is obtained as a result of your participation in this research will be kept as confidential as legally possible. Your research records, just like hospital records, may be subpoenaed by court order or may be inspected by the study sponsor or federal regulatory authorities without your additional consent.

In addition, there are certain instances where the researcher is legally required to give information to the appropriate authorities. These would include mandatory reporting of infectious diseases, mandatory reporting of information about behavior that is imminently dangerous to you or to others, such as suicide, child abuse, etc.

In any publications that result from this research, neither your name nor any information from which you might be identified will be published without your consent.

\section{Who can I talk to if I have questions or concerns?}

If you have any questions or concerns about this research, you can contact Dr. Claire St. Peter at Claire.StPeter@mail.wvu.edu from the Dept. of Psychology at West Virginia University. For information regarding your rights as a participant in research or to talk about the research, contact the WVU Office of Human Research Protection (OHRP) at (304) 293-7073 or by email at IRB@mail.wvu.edu.

\section{Signatures}

You have been given the opportunity to ask questions about the research, and you have received answers concerning areas you did not understand. Upon signing this form, you will receive a copy.

\section{Participant Signature}

I willingly agree to participate in this research.

\author{
Signature of Participant
}


Printed Name

Date

\section{Consenting Individual Signature}

The participant has had the opportunity to have questions addressed. The participant willingly agrees to be in the study.

Signature of Person Obtaining Informed Consent

Printed Name Date




\section{Appendix B: Demographics Survey}

What is your date of birth?

What is your gender?

What is your ethnicity?

What is your annual income?

Are you currently a WVU student? (check one)

Yes _ _ No _ _ Prefer not to say

$\underline{\text { Are you currently taking any medications? (check one) }}$

_ Yes__ No _ _ Prefer not to say

Have you been diagnosed with any of the following learning disabilities (check all that apply; check "None" if none apply):

_ None _ I'd rather not say

_Dyscalculia __ Dysgraphia __ Dyslexia _ ADHD _ D _ Dyspraxia

_ Non-verbal Learning Disabilities____ _ Executive Functioning Disabilities

_Oral/Written Language Disorder and Specific Reading Comprehension Deficit

If yes, what medications are you currently taking? What is the dose and timing of your medication?

Do you have a color-vision deficiency?

Y Yes__ No _ _ Prefer not to say

If yes, what kind? 


\section{Appendix C: Experiment 1 Stimuli}

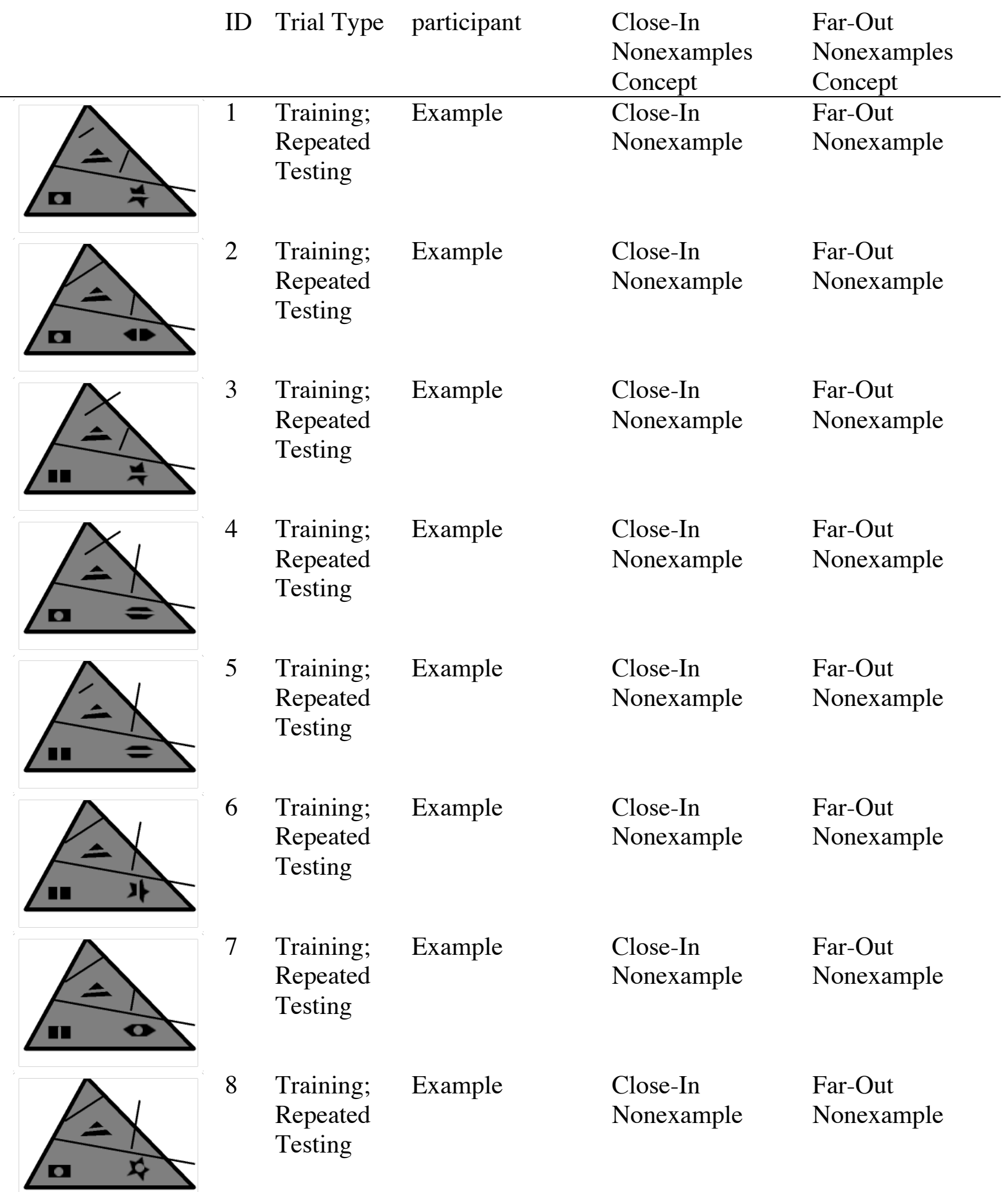



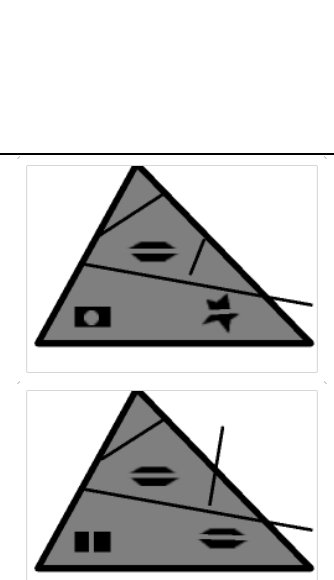

ID Trial Type participant

Close-In

Far-Out

Nonexamples

Nonexamples

Concept

Concept

9 Training;

Example

Close-In

Far-Out

Repeated

Nonexample

Nonexample

Testing

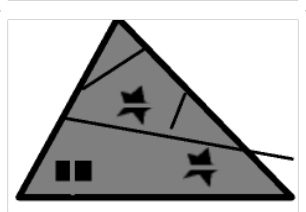

11 Training;

Example

Close-In

Far-Out

Repeated

Nonexample

Far-Out

Close-In

Nonexample

Testing

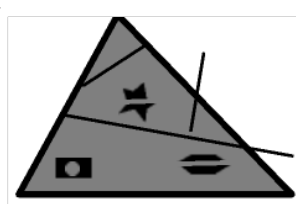

\section{Training; Example}

Repeated

Nonexample

Testing

Close-In

Nonexample

Far-Out

Nonexample

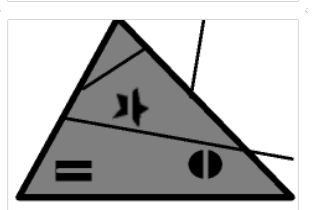

13 Training; Example

Close-In

Nonexample

Example

Repeated

Testing

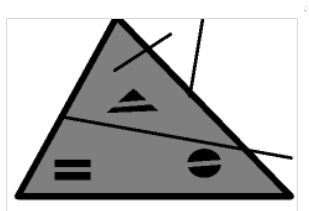

14 Training;

Example

Close-In

Example

Repeated

Nonexample

Testing

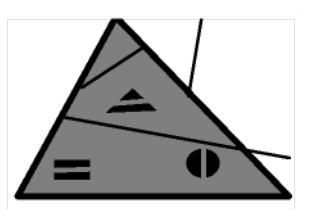

15 Training;

Example

Close-In

Example

Repeated

Nonexample

Testing

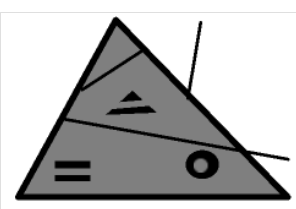

16 Training;

Repeated

Example

Close-In

Example

Testing

Nonexample

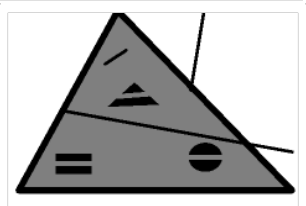

17 Training;

Repeated

Close-In

Nonexample

\section{Example}

Testing 

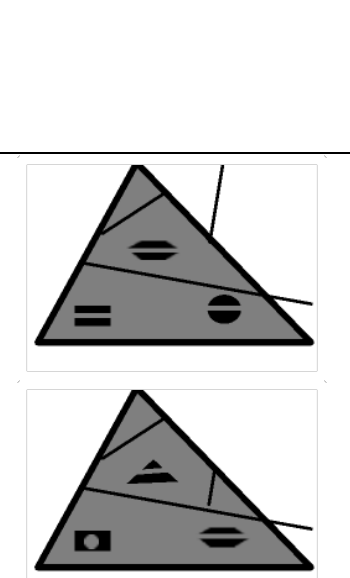

ID Trial Type participant

Close-In

Far-Out

Nonexamples

Nonexamples

Concept

Concept

18 Training;

Close-In

Example

Repeated

Nonexample

Testing

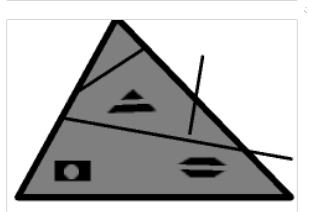

20

19 Training;

Example

Example

Far-Out

Repeated

Nonexample

Testing

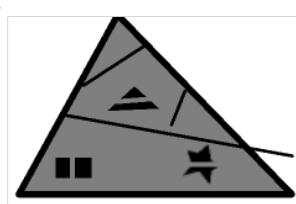

21 Training;

Example

Repeated

Example

Example

Far-Out

Repeated

Nonexample

Testing

Testing

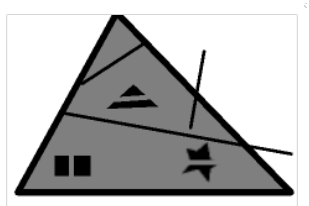

22

Training;

Example

Example

Far-Out

Testing

Example

Far-Out

Nonexample

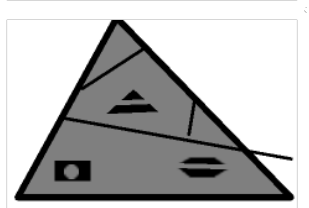

23 Training;

Example

Repeated

Example

Nonexample

Testing

24 Training;

Example

Example

Far-Out

Repeated

Testing

25 Untrained

Testing

(Far-Out)

Example

Far-Out

Far-Out

Nonexample

Far-Out

Nonexample

Nonexample

26 Untrained

Testing

Example

Far-Out

Nonexample

Far-Out

Nonexample 

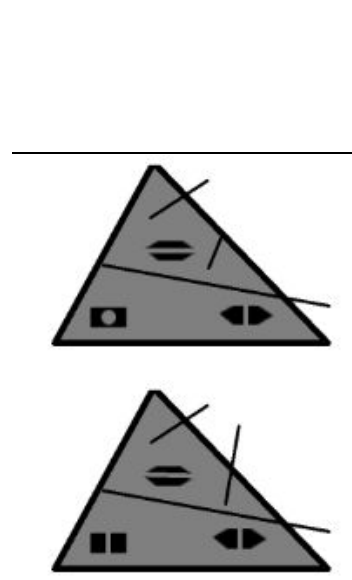

ID Trial Type participant

Close-In

Far-Out

Nonexamples

Nonexamples

Concept

Concept

27 Untrained Example

Far-Out

Far-Out

Testing

(Far-Out)

Nonexample

Nonexample

28 Untrained

Testing

(Far-Out)

Example

Far-Out

Far-Out

Nonexample

Nonexample

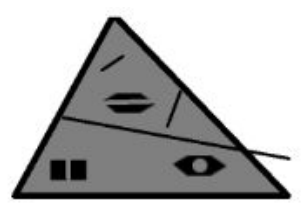

29 Untrained

Example

Far-Out

Far-Out

Testing

(Far-Out)

Nonexample

Nonexample

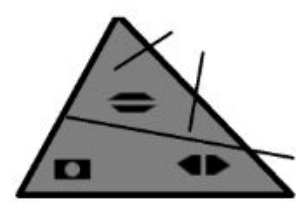

30 Untrained

Testing

(Far-Out)

Example

Far-Out

Nonexample

Far-Out

Nonexample

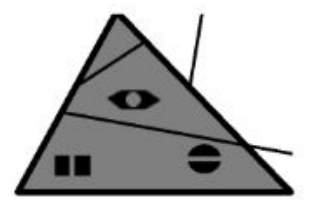

31 Untrained

Example

Close-In

Close-In

Testing

(Close-In)

Nonexample

Nonexample

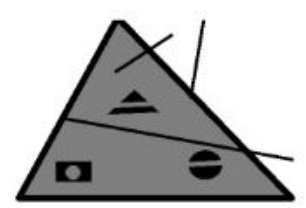

32 Untrained

Testing

(Close-In)

Example

Close-In

Close-In

Nonexample

Nonexample

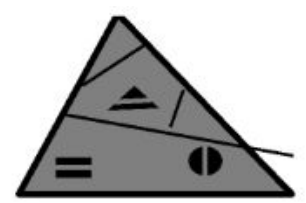

33 Untrained

Example

Close-In

Close-In

Testing

(Close-In)

Nonexample

Nonexample

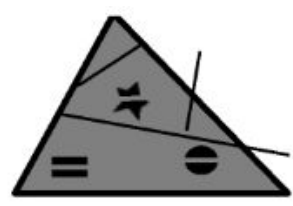

34 Untrained

Example

Close-In

Close-In

Testing

(Close-In)

Nonexample

Nonexample

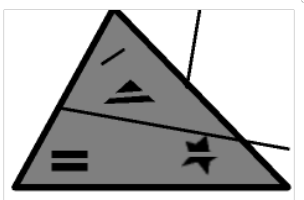

35 Untrained

Testing

(Close-In)

\section{Example}

Close-In

Close-In

Nonexample

Nonexample 

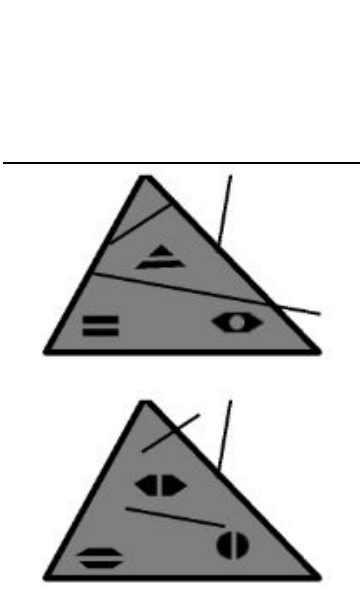
37 Untrained Far-Out
Testing Nonexample
(Far-Out)

ID Trial Type participant

Close-In

Far-Out

Nonexamples

Nonexamples

Concept

Concept

36 Untrained Example

Close-In

Close-In

Testing

(Close-In)

Nonexample

Nonexample

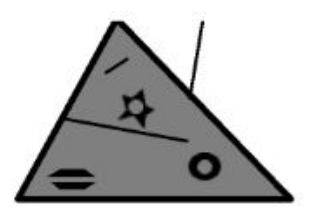

38 Untrained

Far-Out

Testing

Nonexample

(Far-Out)

Example

Far-Out

Nonexample

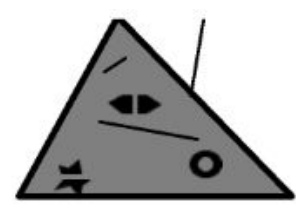

39 Untrained Far-Out

Testing Nonexample

Example

Far-Out

(Far-Out)

Example

Far-Out

Nonexample

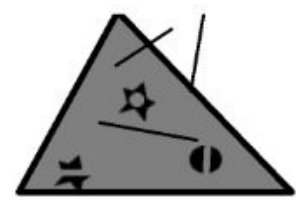

40

$\begin{array}{ll}\text { Untrained } & \text { Far-Out } \\ \text { Testing } & \text { Nonexample }\end{array}$

Example

Far-Out

(Far-Out)

Nonexample

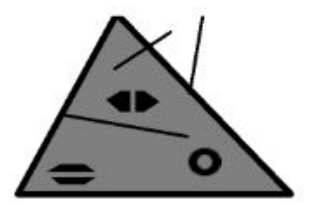

41 Untrained

Far-Out

Testing

Nonexample

(Far-Out)

Example

Far-Out

Nonexample

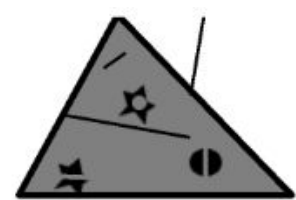

42 Untrained

Far-Out

Testing Nonexample

(Far-Out)

Example

Far-Out

Nonexample

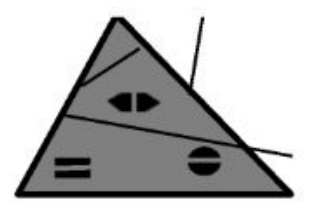

43 Untrained

Close-In

Testing

(Close-In)

Nonexample

Example

Close-In

Nonexample

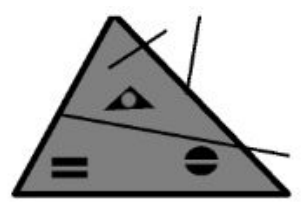

44 Untrained

Testing

Close-In

Nonexample

Example

Close-In

Nonexample 


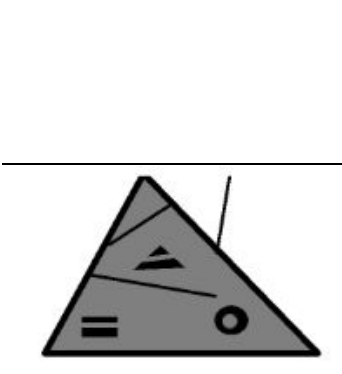

ID Trial Type participant

Close-In

Far-Out

Nonexamples

Nonexamples

Concept

Concept

45 Untrained Close-In

Testing

Nonexample

Example

Close-In

(Close-In)

Nonexample

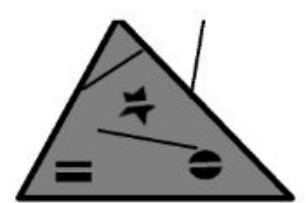

46 Untrained

Testing Nonexample

Example

Close-In

(Close-In)

Close-In

Nonexample

Nonexample

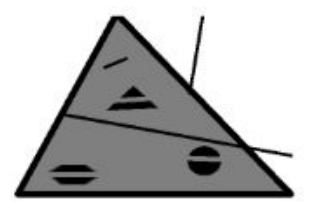

47

$\begin{array}{ll}\text { Untrained } & \text { Close-In } \\ \text { Testing } & \text { Nonexample } \\ \text { (Close-In) } & \end{array}$

Example

Close-In

(Close-In)

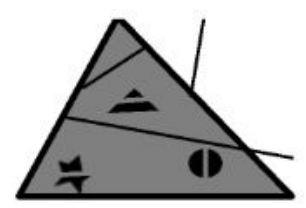

48 Untrained

Close-In

Testing

Nonexample

Example

Close-In

(Close-In)

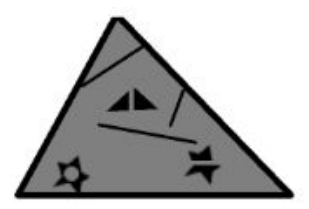

49

$\begin{array}{ll}\text { Untrained } & \text { Far-Out } \\ \text { Testing } & \text { Nonexample }\end{array}$

Far-Out

Example (Far-Out)

Nonexample

Nonexample

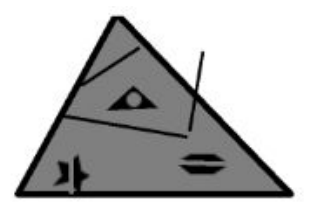

50 Untrained

Far-Out

Testing

Nonexample

(Far-Out)

Far-Out

Nonexample

Example

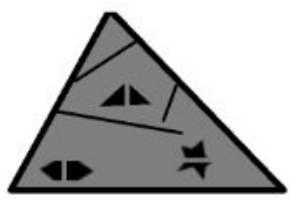

51 Untrained

Far-Out

Testing Nonexample

Far-Out

Example

(Far-Out)

Nonexample

Far-Out

Example

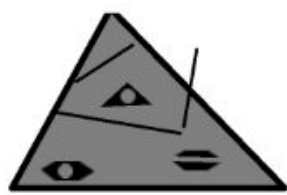

52 Untrained

Far-Out

Nonexample

(Far-Out)

Nonexample

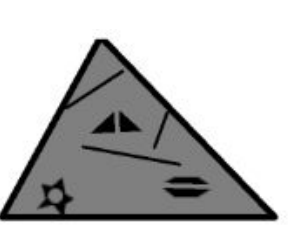

53 Untrained Far-Out

Testing

Nonexample

Far-Out

Nonexample

\section{Example} (Far-Out) 


\begin{tabular}{lllll} 
ID & Trial Type participant & $\begin{array}{l}\text { Close-In } \\
\text { Nonexamples } \\
\text { Concept }\end{array}$ & $\begin{array}{l}\text { Far-Out } \\
\text { Nonexamples } \\
\text { Concept }\end{array}$ \\
\hline & $\begin{array}{llll}\text { Untrained } \\
\text { Testing } \\
\text { (Far-Out) }\end{array}$ & $\begin{array}{l}\text { Far-Out } \\
\text { Nonexample }\end{array}$ & $\begin{array}{l}\text { Far-Out } \\
\text { Nonexample }\end{array}$ & Example \\
& & & &
\end{tabular}

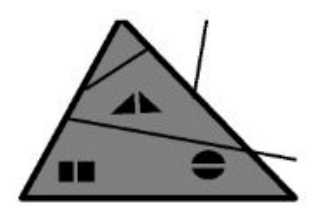

55 Untrained

Testing Nonexample

Close-In

Example

(Close-In)

Nonexample

56 Untrained

Close-In

Testing

Nonexample

Close-In

Nonexample

Example

(Close-In)

57 Untrained Close-In

Testing

Nonexample

Close-In

Nonexample

Example

(Close-In)

58 Untrained

Close-In

Testing

Nonexample

(Close-In)

Close-In

Nonexample

Example

0

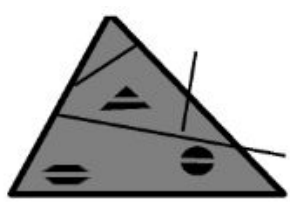

59

Untrained

Close-In

Testing

(Close-In)

Nonexample

Close-In

Nonexample

Example

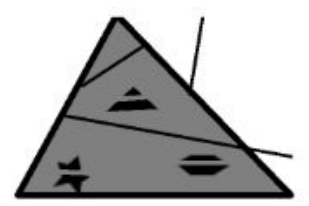

60 Untrained

Close-In

Testing

Nonexample

Close-In

Nonexample

Example

(Close-In)

61 Untrained

Far-Out

Far-Out

Far-Out

Testing

Nonexample

Nonexample

Nonexample

(Far-Out)

62 Untrained

Testing

(Far-Out)
Far-Out
Nonexample

Far-Out

Nonexample

Far-Out

Nonexample 


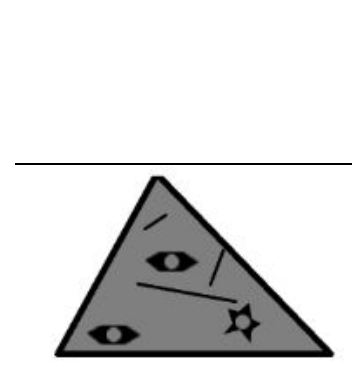

ID Trial Type participant

Close-In

Far-Out

Nonexamples

Nonexamples

Concept

Concept

63 Untrained Far-Out

Far-Out

Far-Out

Testing

Nonexample

Nonexample

Nonexample

(Far-Out)

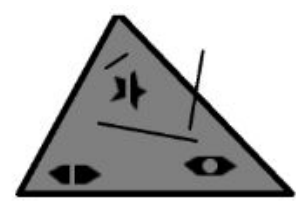

64 Untrained Far-Out

Testing Nonexample

(Far-Out)

Far-Out

Far-Out

Nonexample

Nonexample

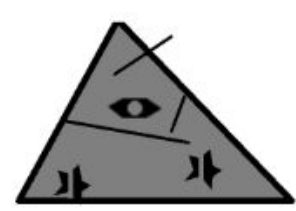

65 Untrained

Far-Out

Far-Out

Far-Out

Testing

Nonexample

(Far-Out)

Nonexample

Nonexample

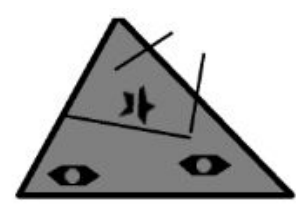

66

Untrained

Far-Out

Nonexample

Far-Out

Nonexample

Far-Out

Testing

(Far-Out)

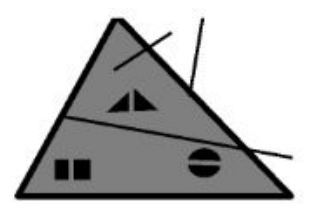

67

Untrained

Close-In

Testing

(Close-In)

Nonexample

Close-In

Close-In

Nonexample

Nonexample

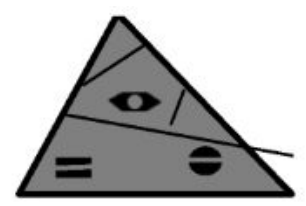

68 Untrained

Close-In

Close-In

Close-In

Testing

(Close-In)

Nonexample

Nonexample

Nonexample

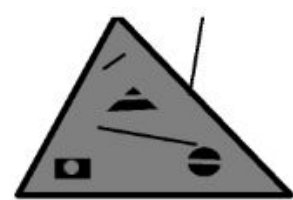

69

Untrained

Close-In

Close-In

Close-In

Testing Nonexample

Nonexample

Nonexample

(Close-In)

70 Untrained

Close-In

Close-In

Close-In

Testing

(Close-In)

Nonexample

Nonexample

71 Untrained

Testing

(Close-In)

Close-In

Close-In

Close-In

Nonexample

Nonexample

Nonexample 


\begin{tabular}{lllll} 
ID & Trial Type participant & $\begin{array}{l}\text { Close-In } \\
\text { Nonexamples } \\
\text { Concept }\end{array}$ & $\begin{array}{l}\text { Far-Out } \\
\text { Nonexamples } \\
\text { Concept }\end{array}$ \\
\hline & $\begin{array}{l}\text { Untrained } \\
\text { Testing } \\
\text { (Close-In) }\end{array}$ & $\begin{array}{l}\text { Close-In } \\
\text { Nonexample }\end{array}$ & $\begin{array}{l}\text { Close-In } \\
\text { Nonexample }\end{array}$ & $\begin{array}{l}\text { Nonexample } \\
\end{array}$ \\
& & &
\end{tabular}




\section{Appendix D: Pre-Experimental Stimuli Evaluations (Experiment 1)}

After the stimuli were made, four rounds of testing were conducted to ensure the stimuli were appropriate for use in this experiment.

First, testing was conducted using a parallel procedure but adapted for pigeons as subjects. To test whether the difference between the relevant feature and each nonrelevant feature that replaced it was salient. To test this, trials were conducted with two stimuli, where responses were made by pecking on a touch screen and correct responses were followed by access to food. The two stimuli presented differed in the presence or absence of a relevant feature (relevant features were replaced with a replacement feature). The location of each feature varied across trials. When responding remained inaccurate, the stimuli were modified so that each feature always appeared at the same place. Despite these changes, responding remained inaccurate and experimentation with pigeons was discontinued.

Second, the same stimuli were used, and tests were conducted to evaluate if the difference between the relevant feature and each replacement feature was salient. To test this, trials were conducted that akin to those described in Trial Structure except containing only two stimuli. The two stimuli differed in exactly one way: the relevant feature was present in one and replaced with one of the nonrelevant features in the other. A single block of 24 trials was conducted for each relevant/nonrelevant feature pairing (nine relevant features $\mathrm{x}$ two nonrelevant replacements $=18$ total pairs). This test was informally conducted with two adult participants who were affiliated with the author but unfamiliar with the experimental question and procedures. Both participants responded accurately during at least 23 of 24 trials across all stimulus pairings, indicating that the difference between the presence and absence of each relevant features was salient. 
Third, two other adults affiliated with the author informally completed the full experiment. Response patterns during baseline suggested one stimulus feature (number of sides) may have been more salient than others. To address this, the features were made to align across the three concepts (as described in Training Stimuli, each concept was changed to be defined by a particular line, geometric object, and geometric-object fill).

Fourth, a third adult completed the experiment with the updated stimuli and responding indicated that each stimulus feature was discriminable and did not suggest a particular feature was more salient than others. These stimuli were used in the present experiment. 


\section{Appendix E: Summary of Procedural Changes Following Pilot Testing}

\begin{tabular}{ccc}
\hline $\begin{array}{c}\text { Pilot Procedure } \\
\text { (Experiments to which this } \\
\text { change applied) }\end{array}$ & $\begin{array}{c}\text { Current Experiment } \\
\text { Procedure }\end{array}$ & Rationale \\
\hline
\end{tabular}

Number of distinct stimuli presented in each trial block gradually increased from 4 to 24 across training blocks. (Exp. 1 and 2)
Training blocks contain all

24 stimuli from the start.
An experiment conducted as an undergraduate capstone compared gradually increasing the number of stimuli and using the full number of stimuli from the start. Results of this experiment suggested acquisition occurred as frequently when the full number of stimuli were used and doing so tended to save time. Effectively, this change shortened training duration.

Money should be delivered following testing because all test trials were followed by neutral feedback, so participants were not able to earn money during testing. The amount was decreased from $\$ 5.00$ to $\$ 2.50$ because this money was delivered a total of six times per participant and, based on pilot testing earnings, $\$ 5.00$ would result in too great $(>\$ 20.00)$ of earnings per hour.

\begin{tabular}{lll}
\hline $\begin{array}{l}\text { Affect was not measured. } \\
\text { (Exp. 1 and 2) }\end{array}$ & $\begin{array}{l}\text { Affect was measured } \\
\text { following each training } \\
\text { condition. }\end{array}$ & $\begin{array}{l}\text { A measure of affect will allow } \\
\text { emotional responses to each } \\
\text { condition to be compared, which } \\
\text { may inform the use of these } \\
\text { procedures in the classroom. }\end{array}$ \\
\hline $\begin{array}{l}\text { Positive or negative } \\
\text { feedback continued to be } \\
\text { given following training } \\
\text { trials repeated during test } \\
\text { trials }(50 \% \text { of test trials were } \\
\text { followed by positive or } \\
\text { negative feedback). (Exp. 1) }\end{array}$ & $\begin{array}{l}\text { Neutral feedback was } \\
\text { given following all test } \\
\text { trials. }\end{array}$ & $\begin{array}{l}\text { Providing feedback during } \\
\text { training trials repeated during } \\
\text { testing but not during baseline } \\
\text { could affect responding during } \\
\text { testing relative to baseline. }\end{array}$ \\
\hline $\begin{array}{l}\text { Before training ended, 25\% } \\
\text { then 50\% of training trials } \\
\begin{array}{l}\text { were followed by neutral } \\
\text { feedback. Testing only }\end{array}\end{array}$ & $\begin{array}{l}\text { Neutral feedback was } \\
\text { provided on all training } \\
\text { trials. Instructions were } \\
\text { given to the participant }\end{array}$ & $\begin{array}{l}\text { This procedure was originally } \\
\text { used to align the method between } \\
\text { human and nonhuman } \\
\text { experiments. Because the }\end{array}$ \\
\hline
\end{tabular}




\begin{tabular}{|c|c|c|}
\hline $\begin{array}{l}\text { began after responding was } \\
\text { accurate even when } 50 \% \text { of } \\
\text { trials were followed by } \\
\text { neutral feedback (akin to the } \\
\text { testing arrangement, see } \\
\text { above). (Exp. 1) }\end{array}$ & $\begin{array}{l}\text { prior to testing to let them } \\
\text { know that positive/negative } \\
\text { feedback would no longer } \\
\text { be provided. }\end{array}$ & $\begin{array}{l}\text { nonhuman experiment was } \\
\text { removed, instructions were a way } \\
\text { to save time by removing } \\
\text { unnecessary experimental stages. } \\
\text { Effectively, this change } \\
\text { shortened training duration. }\end{array}$ \\
\hline $\begin{array}{l}\text { Testing could occur } \\
\text { immediately following } \\
\text { training and may occur over } \\
\text { multiple appointments. (Exp. } \\
\text { 1) }\end{array}$ & $\begin{array}{l}\text { A single training block } \\
\text { occurred prior to testing } \\
\text { and all testing was } \\
\text { completed in one } \\
\text { appointment. }\end{array}$ & $\begin{array}{l}\text { This change ensured the time } \\
\text { between training and testing was } \\
\text { equal across participants. }\end{array}$ \\
\hline $\begin{array}{l}\text { A single set of six baseline } \\
\text { blocks was completed at the } \\
\text { start of the experiment. (Exp. } \\
\text { 1) }\end{array}$ & $\begin{array}{l}\text { A set of six baseline blocks } \\
\text { was completed at the start } \\
\text { of each condition (three } \\
\text { total sets). }\end{array}$ & \multirow{2}{*}{$\begin{array}{l}\text { Because the same stimuli were } \\
\text { used in each condition, training } \\
\text { in one condition could affect } \\
\text { responding in the next. } \\
\text { Collecting baseline prior } \\
\text { provides a comparison for each } \\
\text { condition relative to the } \\
\text { beginning of that condition rather } \\
\text { than the beginning of the } \\
\text { experiment. }\end{array}$} \\
\hline $\begin{array}{l}\text { A single baseline block was } \\
\text { competed for each stimulus } \\
\text { set (three blocks total) at the } \\
\text { start of the experiment. (Exp. } \\
\text { 2) }\end{array}$ & $\begin{array}{l}\text { A set of six baseline blocks } \\
\text { was completed at the start } \\
\text { of each condition with the } \\
\text { stimulus set used for the } \\
\text { that condition. }\end{array}$ & \\
\hline $\begin{array}{l}\text { Only trials with untrained } \\
\text { stimuli were included in } \\
\text { baseline blocks. (Exp. 2) }\end{array}$ & $\begin{array}{l}\text { Both trials with training } \\
\text { and untrained stimuli were } \\
\text { included in baseline } \\
\text { blocks. }\end{array}$ & $\begin{array}{l}\text { The presence of trials with } \\
\text { training stimuli could affect } \\
\text { responding. It is easier to } \\
\text { compare baseline and testing } \\
\text { responding by keeping the } \\
\text { number of trials with training and } \\
\text { untrained stimuli constant } \\
\text { between baseline or testing. }\end{array}$ \\
\hline $\begin{array}{l}\text { Participants were not asked } \\
\text { to name the concept or } \\
\text { describe their strategy for } \\
\text { selecting a stimulus. (Exp. 2) }\end{array}$ & $\begin{array}{l}\text { Participants were asked to } \\
\text { name the concept and } \\
\text { describe their strategy } \\
\text { following testing in each } \\
\text { condition. }\end{array}$ & $\begin{array}{l}\text { These descriptions could provide } \\
\text { evidence of particular patterns of } \\
\text { responding that resulted from } \\
\text { each condition. }\end{array}$ \\
\hline
\end{tabular}




\section{Appendix F: Experiment 1 Baseline/Test Trial Examples}

As in the training trials, the same stimuli are displayed on test trials in all conditions. Test trials are divided into three stimulus arrangements: repeated trials (shown in Appendix E), untrained trials with close-in nonexamples (shown on this page), and untrained trials with far-out nonexamples (shown on the following page).

Trials with Untrained Close-In Nonexamples: Examples-Only Condition The three relevant features for an example of participant (correct during examples-only condition) are: 1 . Inside and outside line; 2 . Internal rectangle; 3 . Horizontal line through top geometric figure. Stimulus IV contains all three relevant features, making it an example of participant. Selecting Stimulus IV would be the only correct response. Stimuli I, II, and III contain an inside and outside line and horizontal line through the top geometric figure but lack an inside and outside line. Because Stimuli I, II, and III each lack one relevant feature, they are close-in nonexamples of participant.

\section{Trials with Untrained Close-In Nonexamples: Close-In Nonexamples Condition} The three relevant features for an example of Close-In Nonexamples concept (correct during Close-In Nonexamples condition) are: 1 . Inside line; 2 . Internal triangle; 3 . Horizontal line through bottom-right geometric figure. Stimulus II contains all three relevant features, making it an example of Close-In Nonexamples concept. Selecting Stimulus II would be the only correct response. Stimulus I contain an inside line and a horizontal line through the bottom-right geometric figure but lacks an inside triangle. Stimuli III and IV contain inside lines and inside triangle but lack a horizontal line through the bottom-right geometric figure. Because Stimuli I, III, and IV each lack one relevant feature, they are close-in nonexamples of Close-In Nonexamples concept.

\section{Trials with Untrained Close-In Nonexamples: Far-Out Nonexamples Condition}

The three relevant features for an example of Far-Out Nonexamples concept (correct during FarOut Nonexamples condition) are: 1 . Outside line; 2. Internal oval; 3 . Horizontal line through bottom-left geometric figure. Stimulus III contains all three relevant features, making it an example of Far-Out Nonexamples concept. Selecting Stimulus III would be the only correct response. Stimuli I, II, and IV contain an outside line and horizontal line through the bottom-left geometric figure but lack an internal oval. Because Stimuli I, II, and IV each lack one relevant feature, they are close-in nonexamples of Close-In Nonexamples concept.

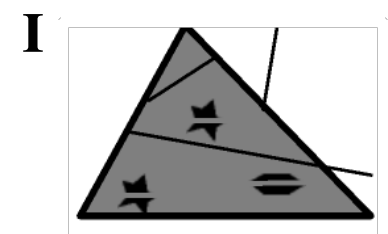

II

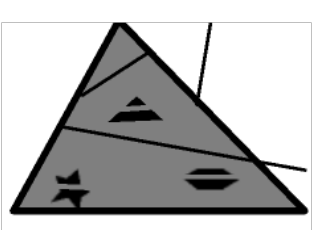

III

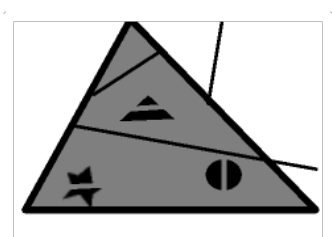

*Roman numerals will not appear on screen and are for reference only. 
Trials with Untrained Far-Out Nonexamples: Examples-Only Condition The three relevant features for an example of participant (correct during examples-only condition) are: 1 . Inside and outside line; 2 . Internal rectangle; 3 . Horizontal line through top geometric figure. Stimulus I contains all three relevant features, making it an example of participant. Selecting Stimulus III would be the only correct response. Stimuli II, III, and IV lack all three relevant features, making them far-out nonexamples of participant.

\section{Trials with Untrained Far-Out Nonexamples: Close-In Nonexamples Condition} The three relevant features for an example of Close-In Nonexamples concept (correct during Close-In Nonexamples condition) are: 1 . Inside line; 2. Internal triangle; 3 . Horizontal line through bottom-right geometric figure. Stimulus III contains all three relevant features, making it an example of Close-In Nonexamples concept. Selecting Stimulus III would be the only correct response. Stimuli I, II, and IV lack all three relevant features, making them far-out nonexamples of Close-In Nonexamples concept.

Trials with Untrained Far-Out Nonexamples: Far-Out Nonexamples Condition The three relevant features for an example of Far-Out Nonexamples concept (correct during FarOut Nonexamples condition) are: 1 . Outside line; 2. Internal oval; 3. Horizontal line through bottom-left geometric figure. Stimulus II contains all three relevant features, making it an example of Far-Out Nonexamples concept. Selecting Stimulus II would be the only correct response. Stimuli I, III, and IV lack all three relevant features, making them far-out nonexamples of Far-Out Nonexamples concept.
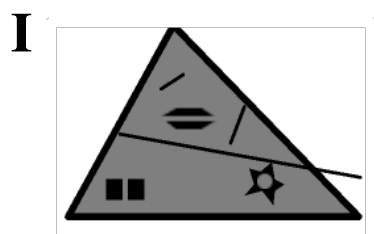

III

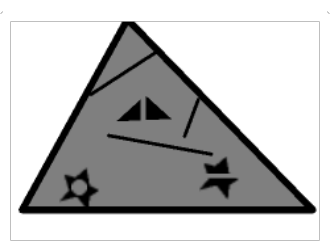

II

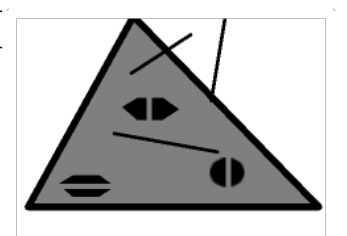

*Roman numerals will not appear on screen and are for reference only. 


\section{Appendix G: Experiment 1 Training Trials Examples}

Note that, as in the experiment, the same stimuli are used during trials in all conditions.

\section{Representative Training Trial Stimuli: Examples-Only Condition}

The three relevant features for an example of participant (correct during examples-only condition) are: 1 . Inside and outside line; 2 . Internal rectangle; 3 . Horizontal line through top geometric figure. Although these stimuli vary in many ways, all four stimuli contain these three features, making them all examples of participant. Selecting any of the four stimuli would be a correct response.

\section{Representative Training Trial Stimuli: Close-In Nonexamples Condition} The three relevant features for an example of Close-In Nonexamples concept (correct during Close-In Nonexamples condition) are: 1. Inside line; 2. Internal triangle; 3. Horizontal line through bottom-right geometric figure. Stimulus II contains three relevant features, making it an example of Close-In Nonexamples concept. Selecting Stimulus II would be the only correct response. Stimuli I, III, and IV contain a horizontal line through the bottom-right geometric figure and a triangle but lacks an inside line. Because Stimuli I, III, and IV each lack one relevant feature, they are close-in nonexamples of Close-In Nonexamples concept.

\section{Representative Training Trial Stimuli: Far-Out Nonexamples Condition}

The three relevant features for an example of Far-Out Nonexamples concept (correct during FarOut Nonexamples condition) are: 1 . Outside line; 2. Internal oval; 3. Horizontal line through bottom-left geometric figure. Stimulus III contains all three relevant features, making it an example of Far-Out Nonexamples concept. Selecting Stimulus I would be the only correct response. Stimuli I, II, and IV lack all three relevant features, making them far-out nonexamples of Far-Out Nonexamples concept.
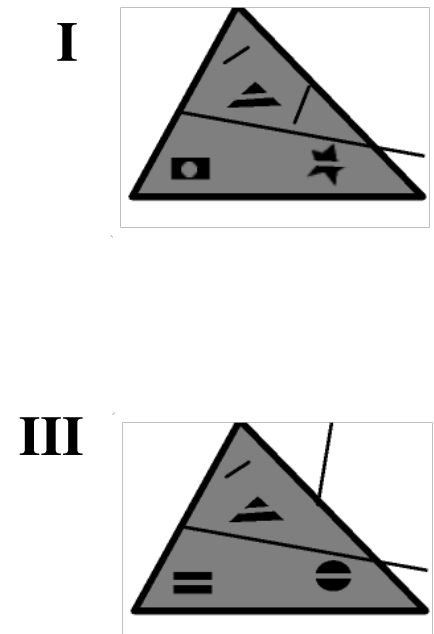

II

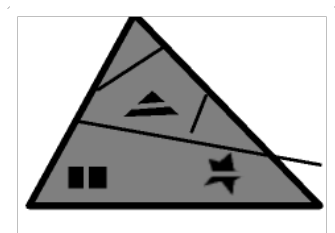

IV

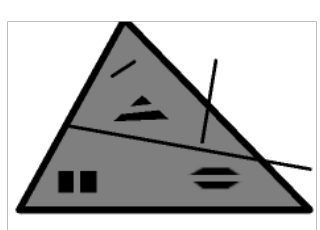

*Roman numerals will not appear on screen and are for reference only. 


\section{Appendix H: PANAS-X}

To what extent have you felt this way while learning this concept:

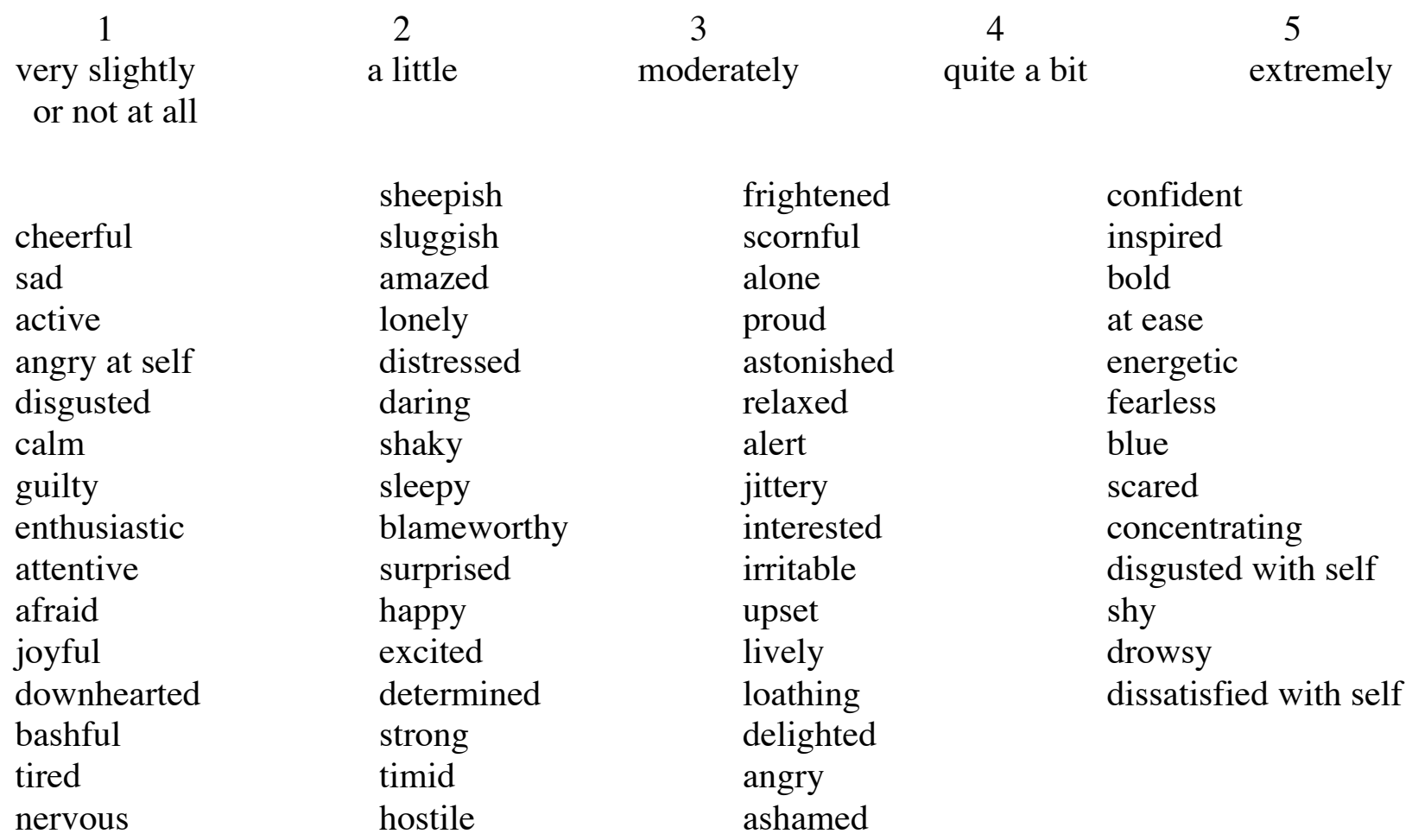

Item Composition of the PANAS-X Scales
General Negative Affect afraid, scared, nervous, jittery, guilty, ashamed, irritable, hostile, upset, distressed
General Positive Affect active, alert, attentive, enthusiastic, excited, inspired, interested, proud, strong, determined

To score a scale, sum the responses to the items in that scale. 


\section{Appendix I: Experiment 1 Incomplete Participants}

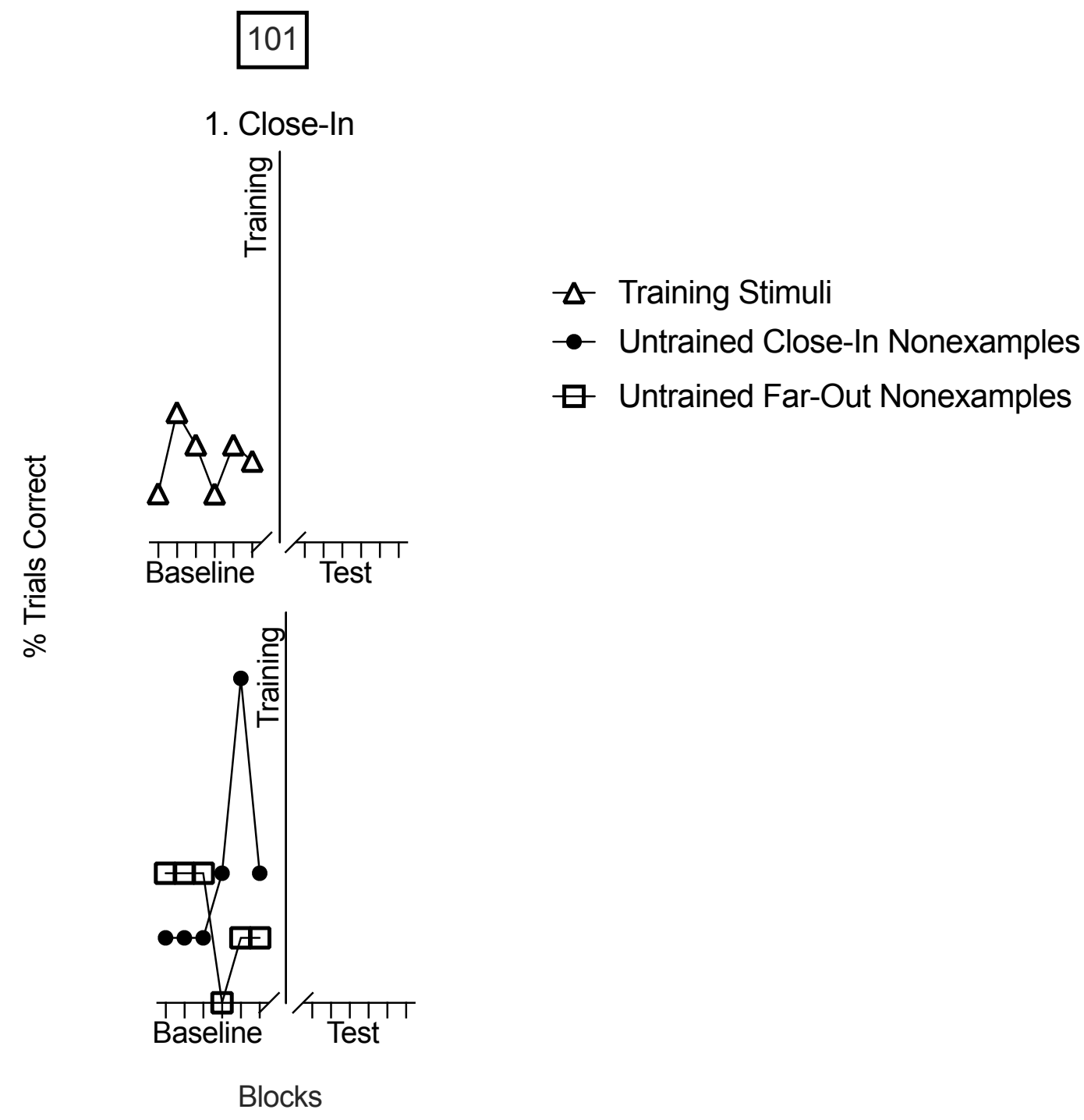

Note. Training blocks are indicated by the solid, vertical line between baseline and testing blocks, though data from these blocks are not displayed. The conditions appear in the order in which that participant experienced them. 


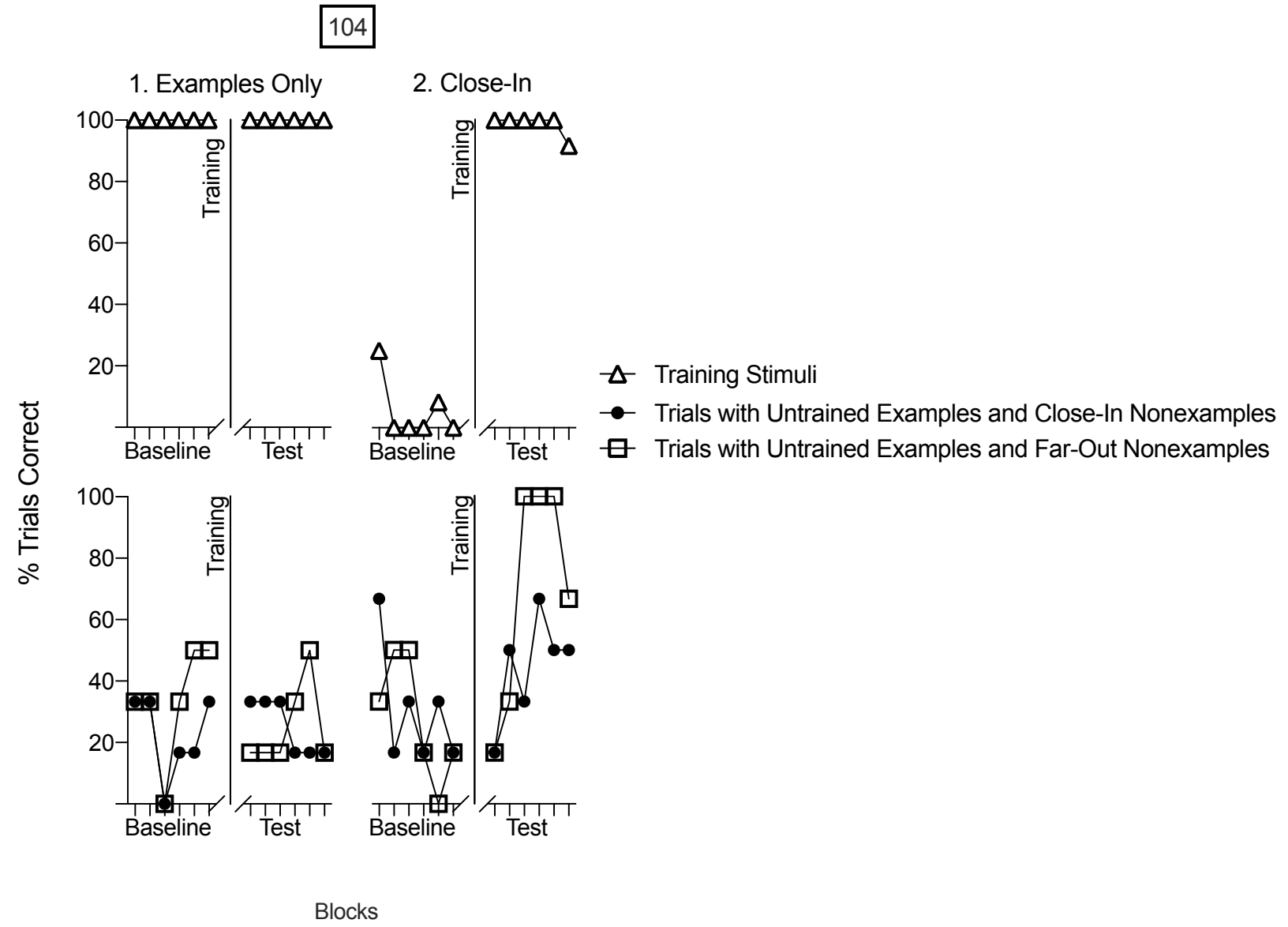

Note. Training blocks are indicated by the solid, vertical line between baseline and testing blocks, though data from these blocks are not displayed. The conditions appear in the order in which that participant experienced them. 


\section{Appendix J: Experiment 2 Stimuli \\ Neurons}

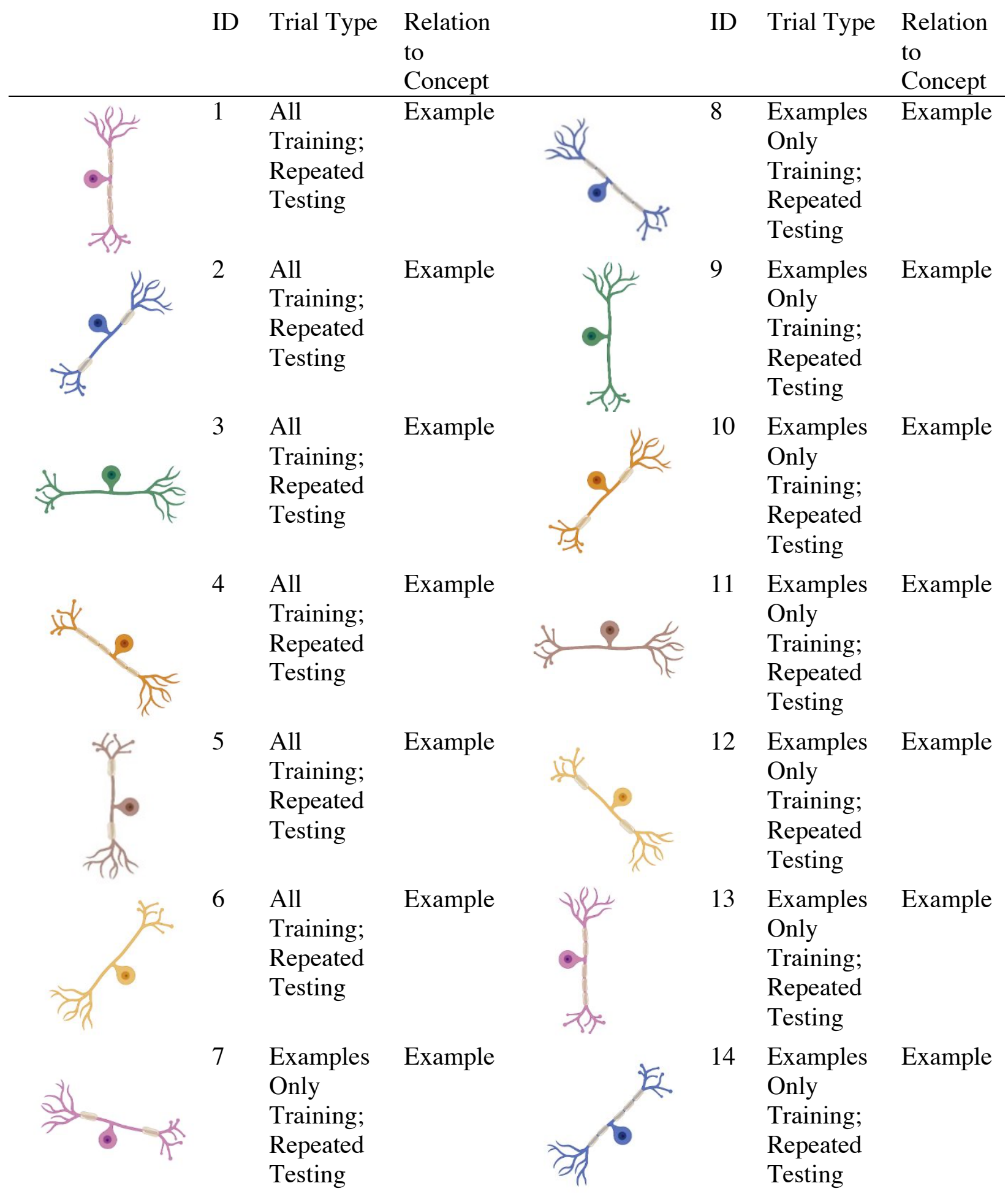




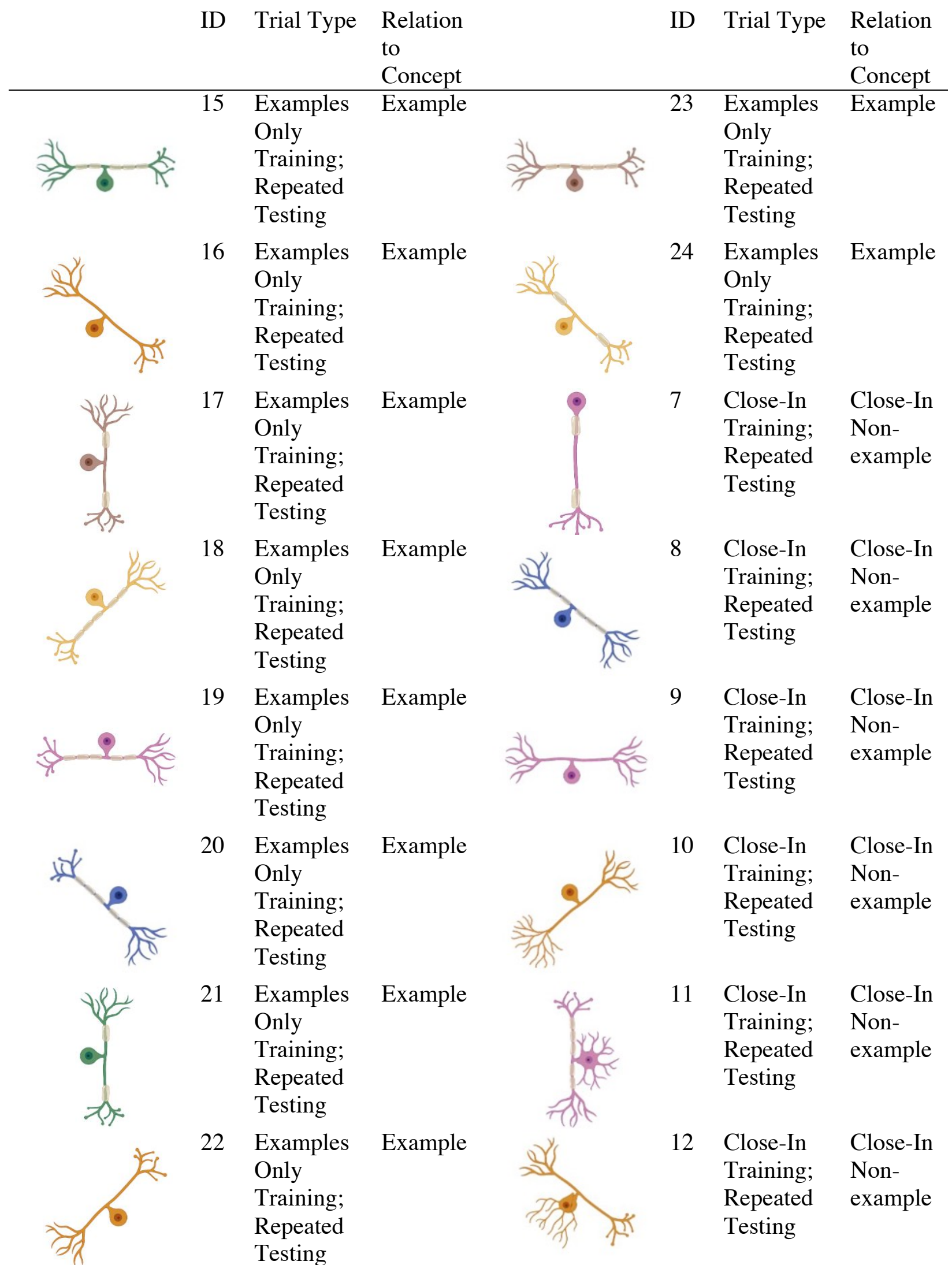




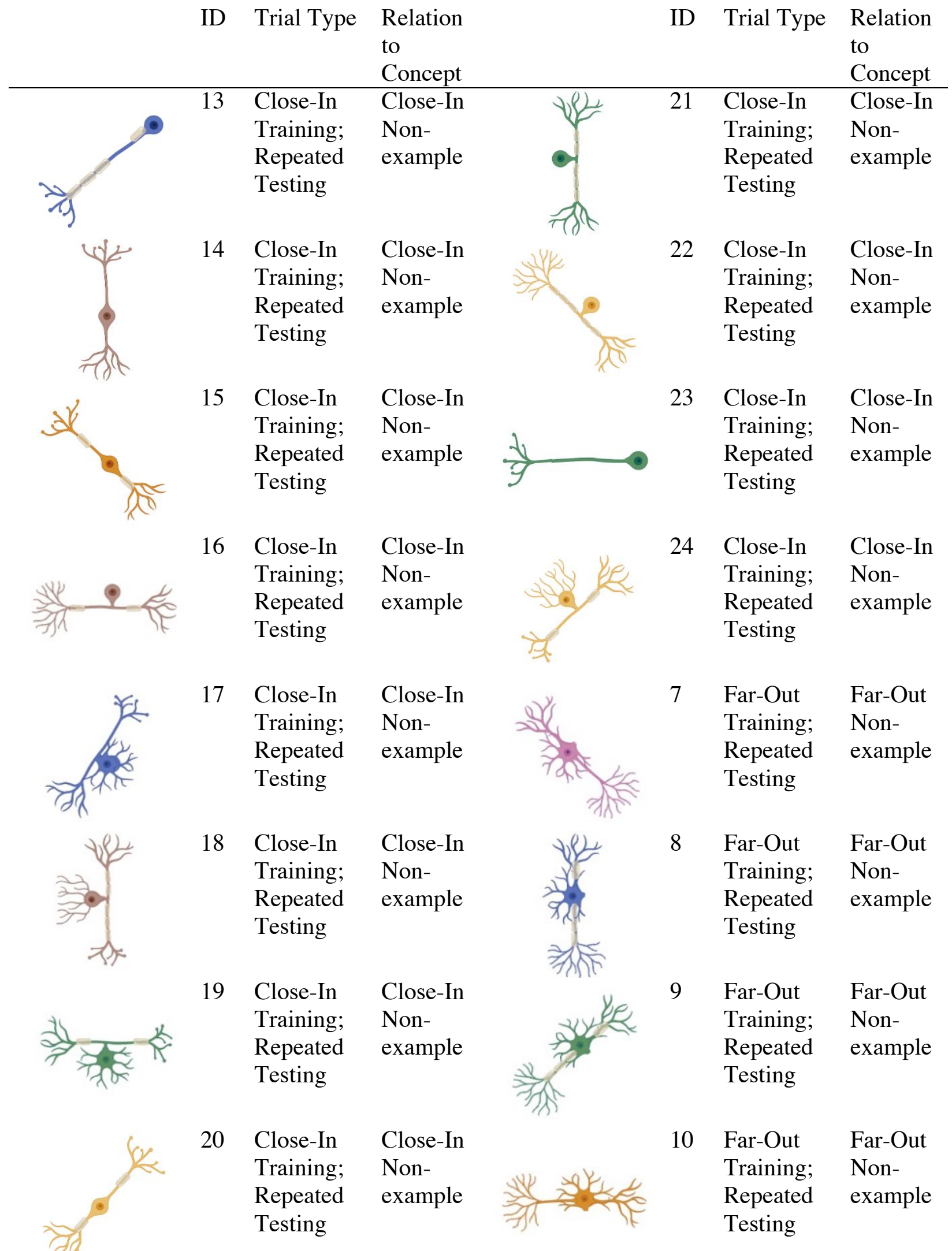




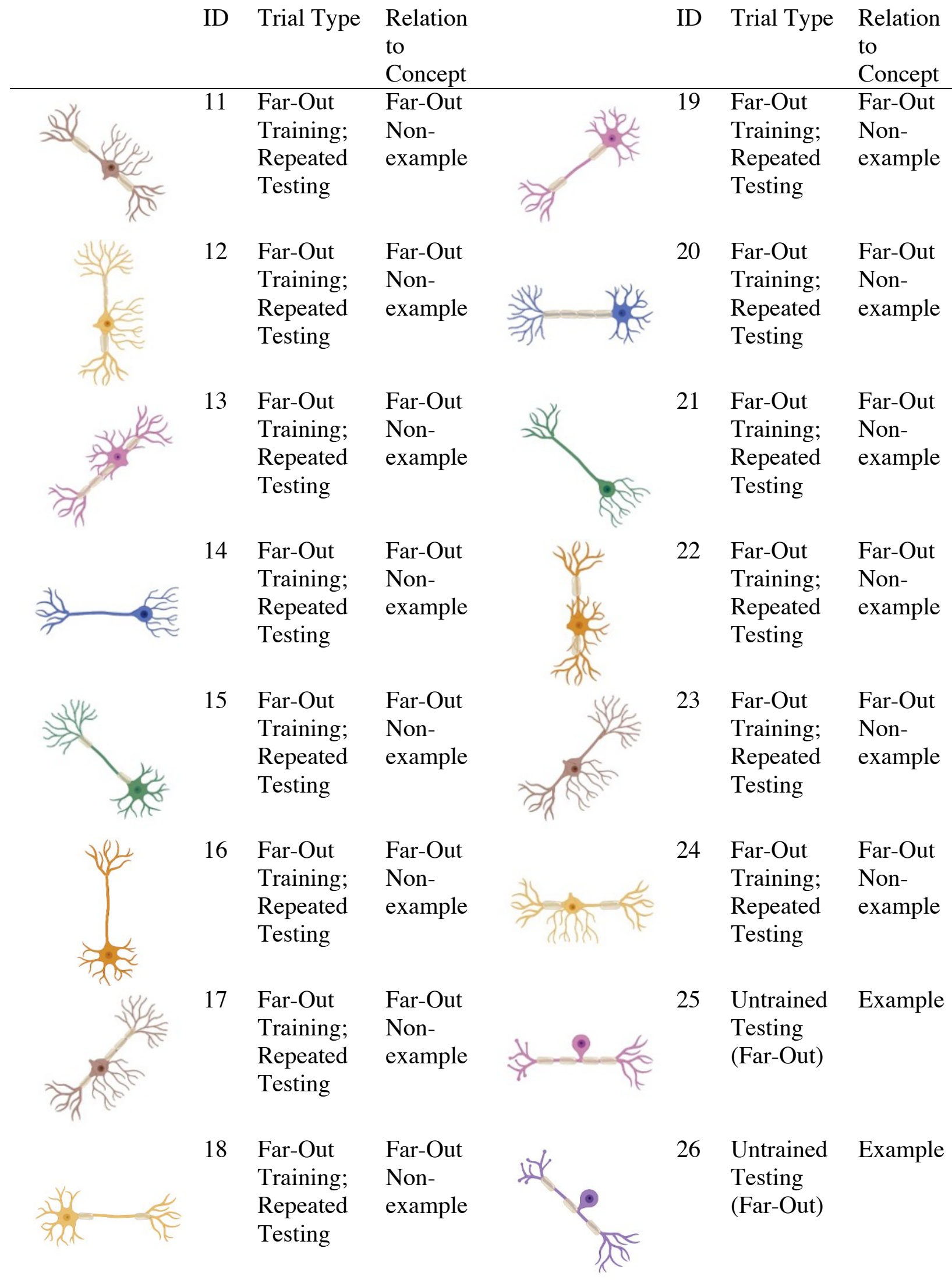




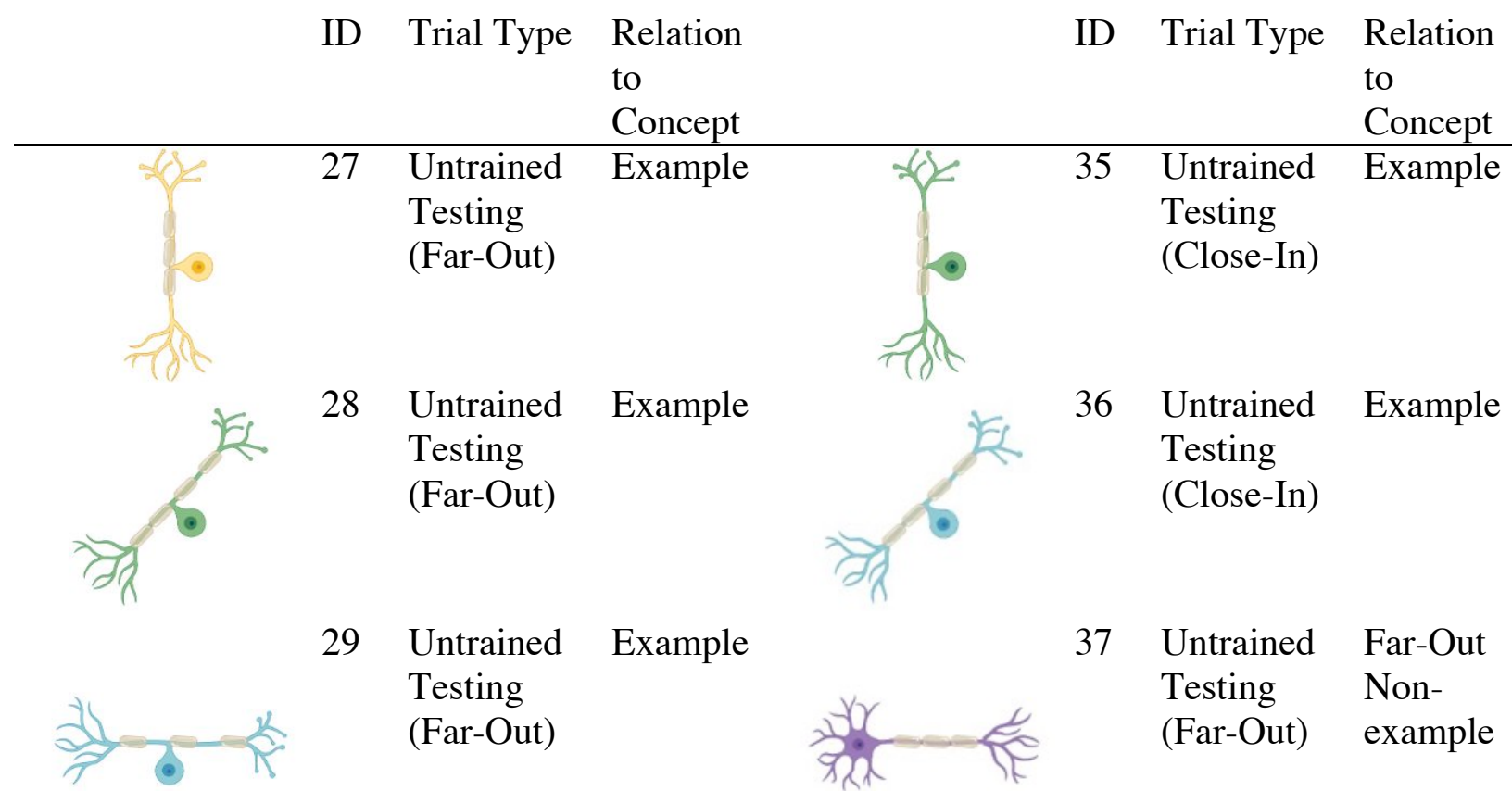

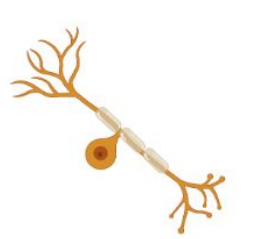

30 Untrained Example

Testing

(Far-Out)

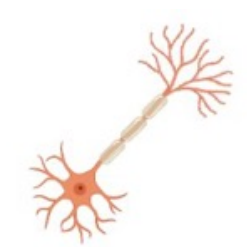

38 Untrained Far-Out Testing Non-

(Far-Out) example

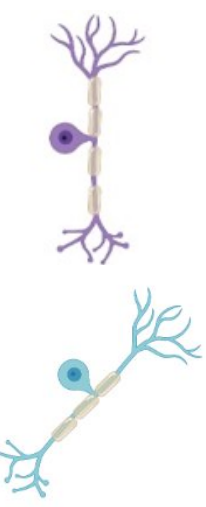

31 Untrained Example

Testing

(Close-In)

32 Untrained Example

Testing

(Close-In)

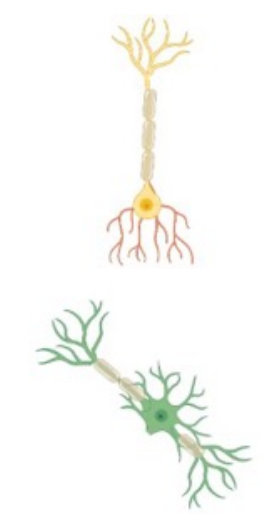

39 Untrained Far-Out

Testing Non-

(Far-Out) example

40 Untrained Far-Out Testing Non-

(Far-Out) example

33 Untrained Example

Testing

(Close-In)

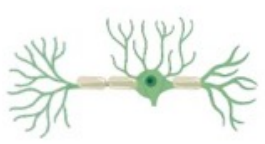

41 Untrained Far-Out

Testing Non-

(Far-Out) example

34 Untrained Example

Testing

(Close-In)

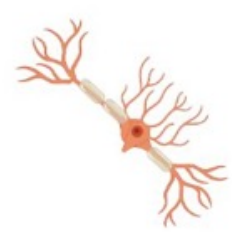

42 Untrained Far-Out

Testing Non-

(Far-Out) example 


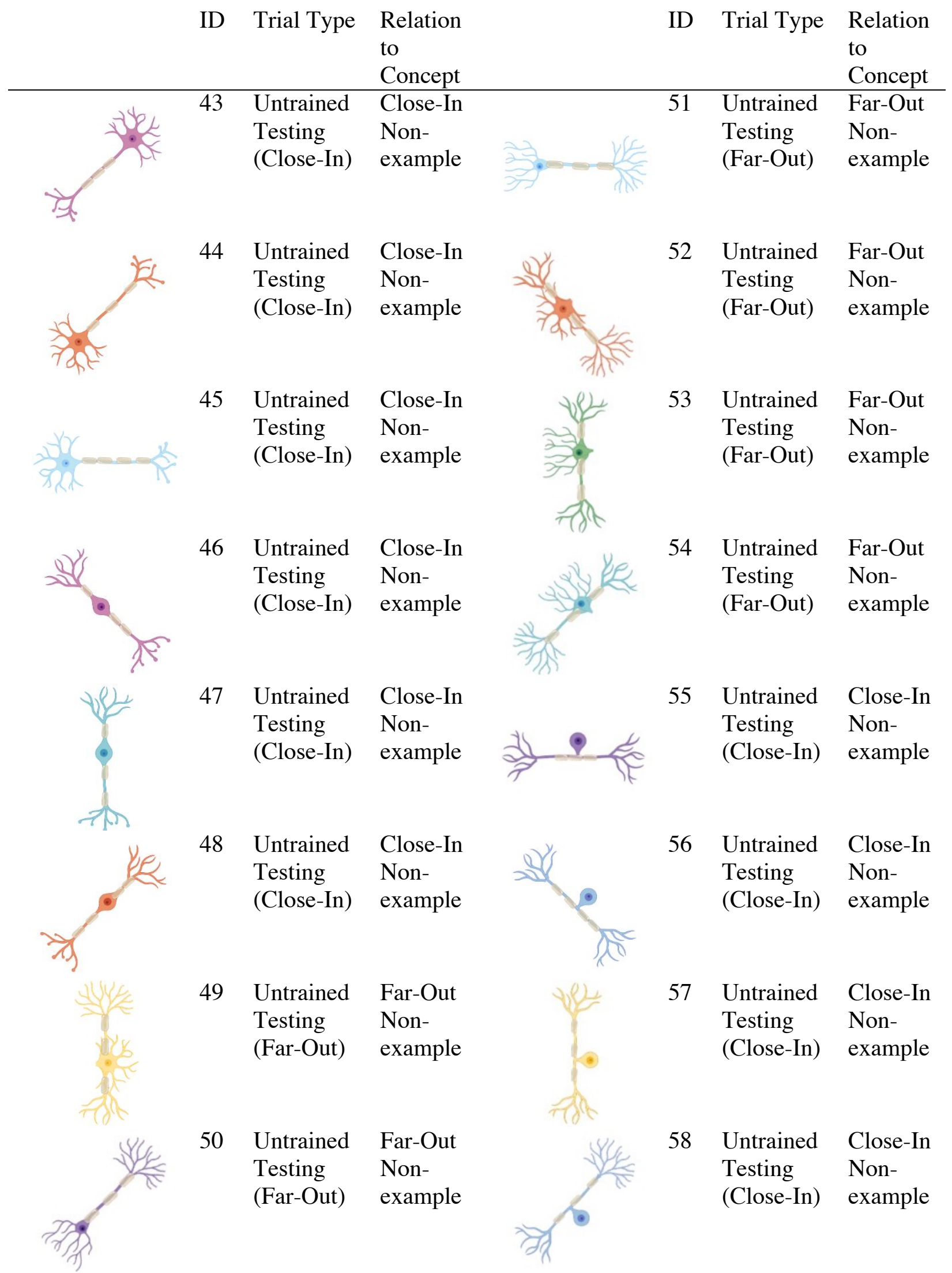



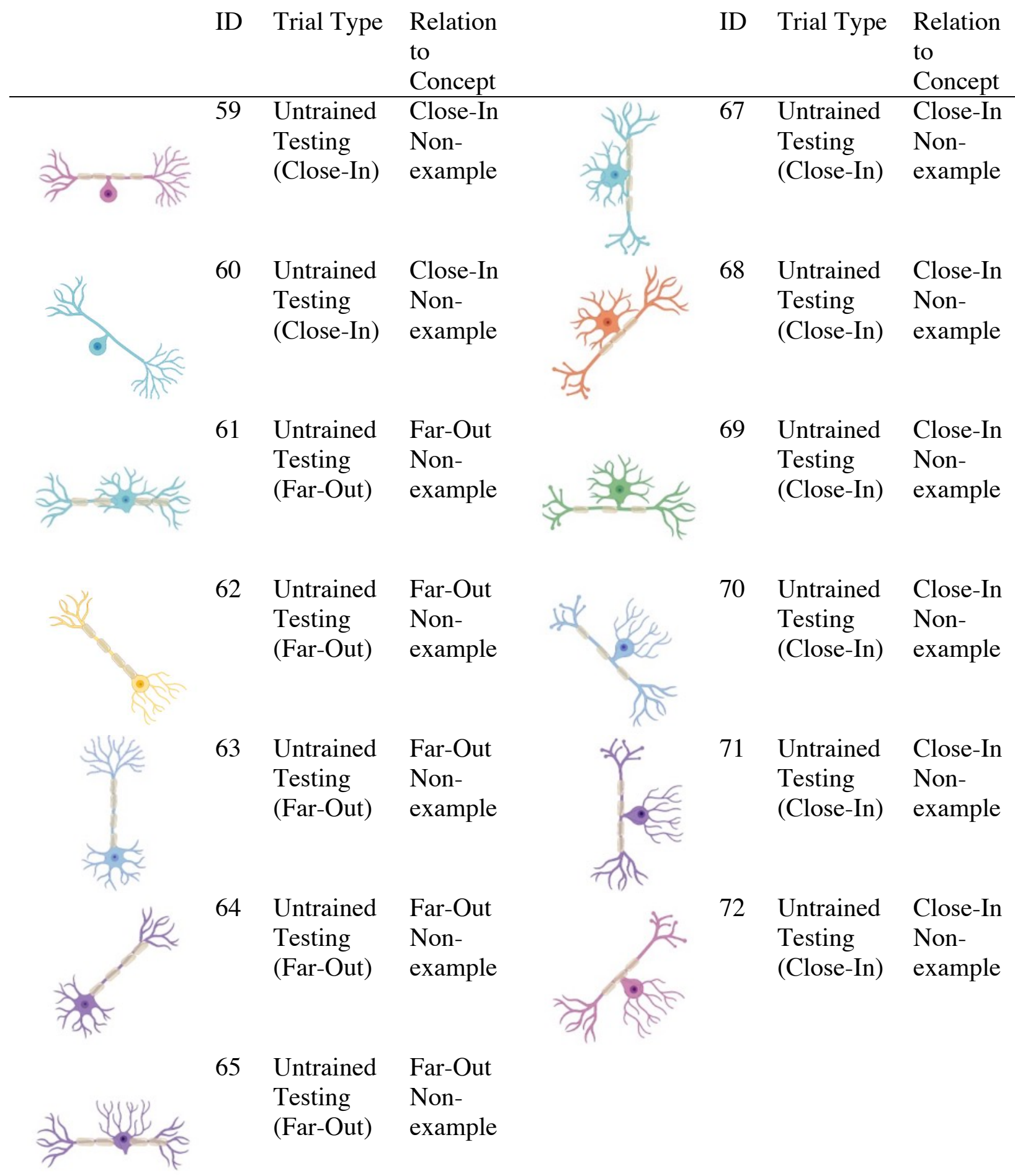

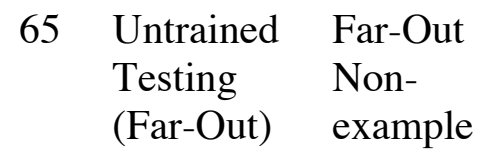

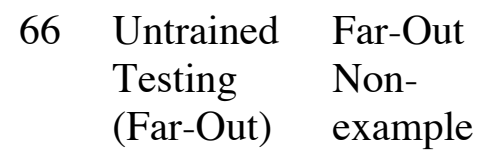




\section{Bacteria Cells}

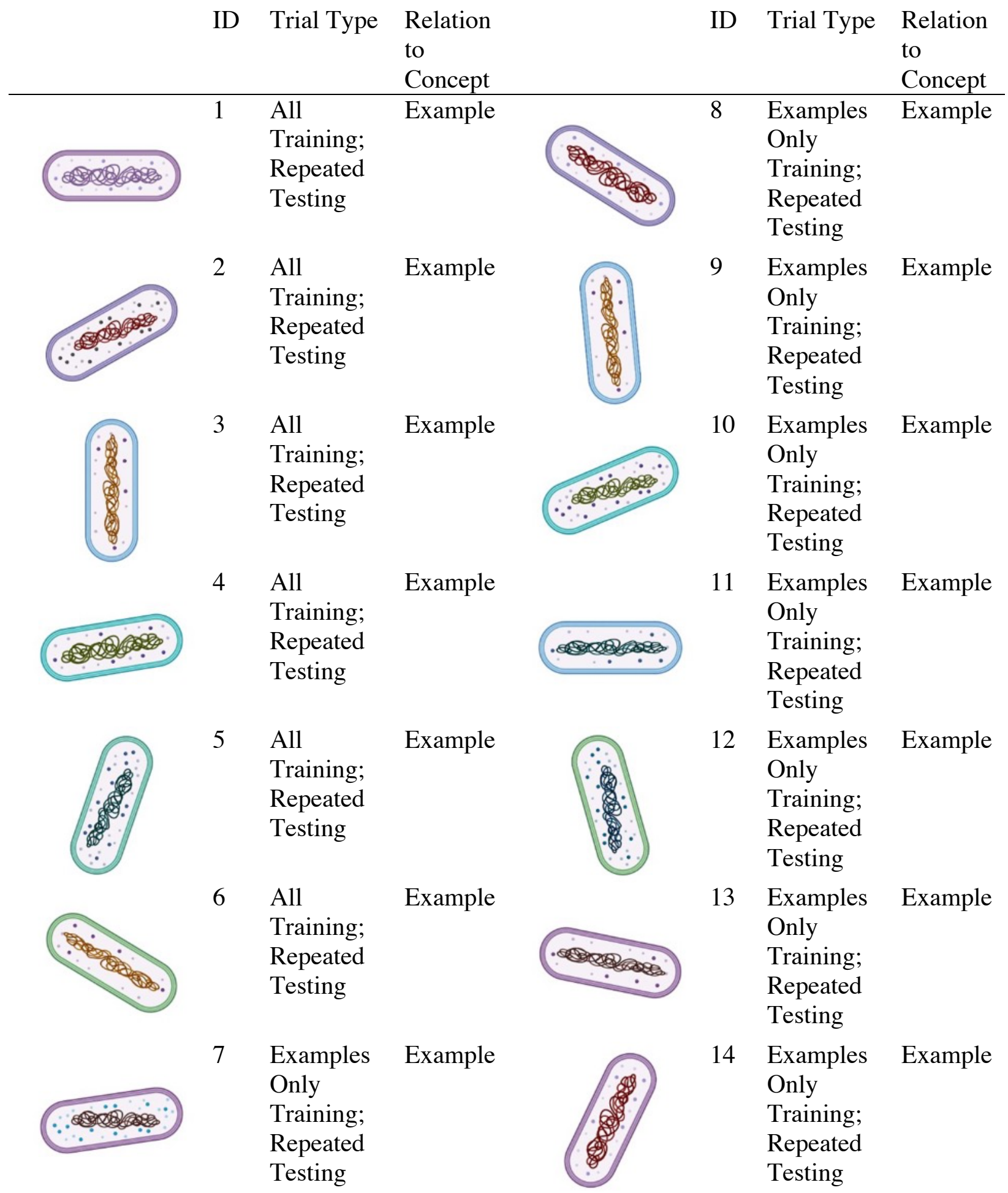




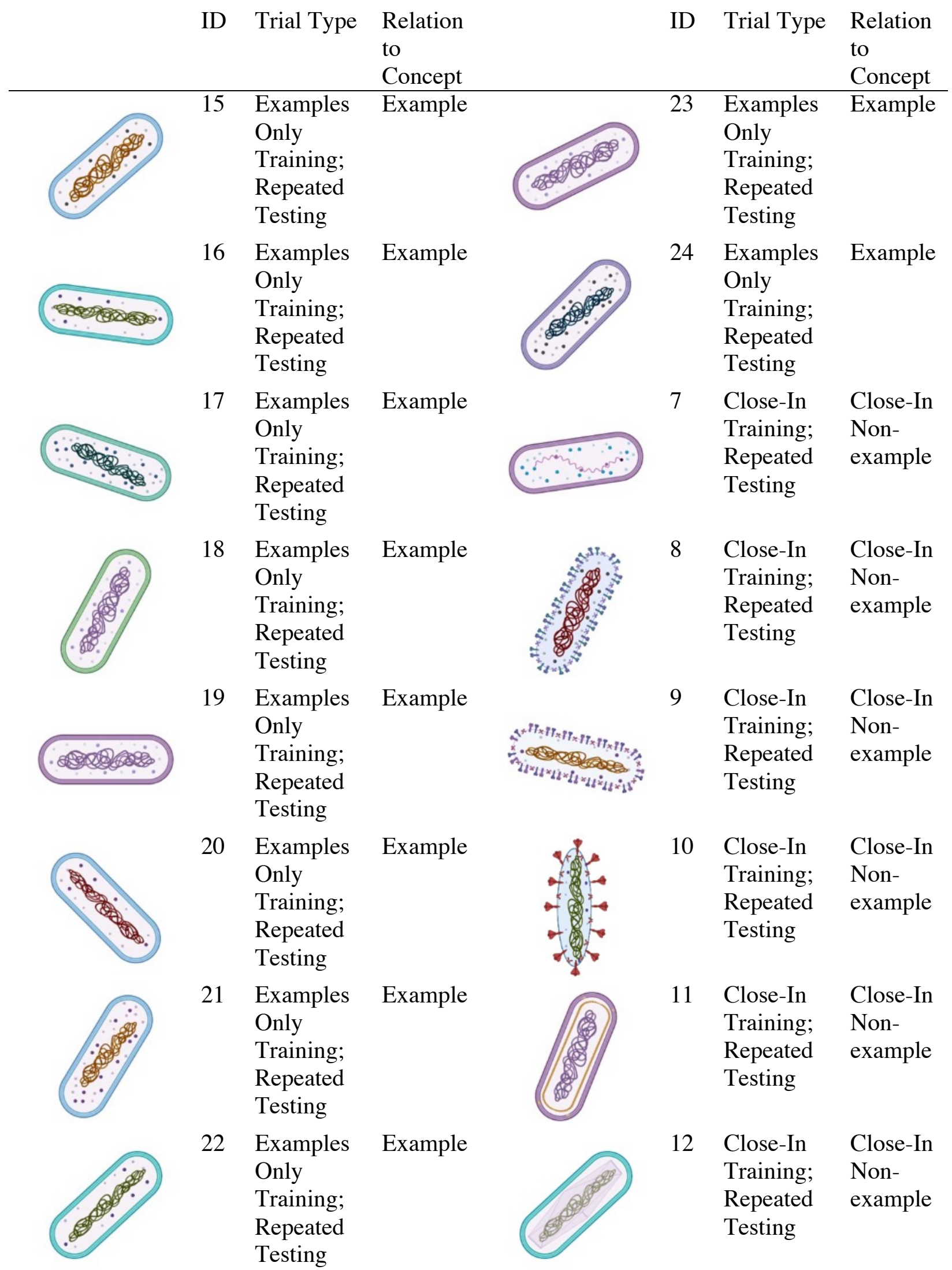




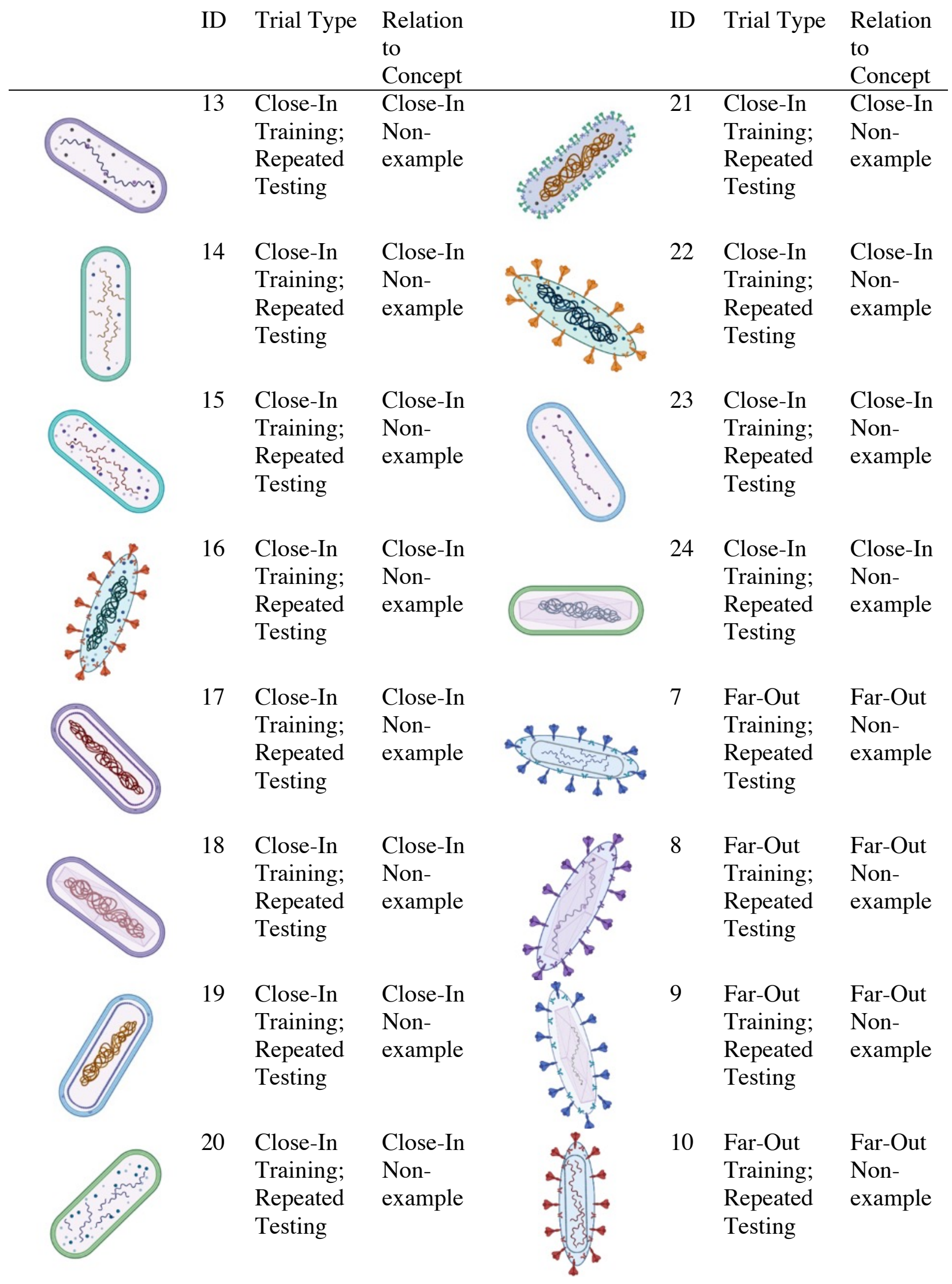




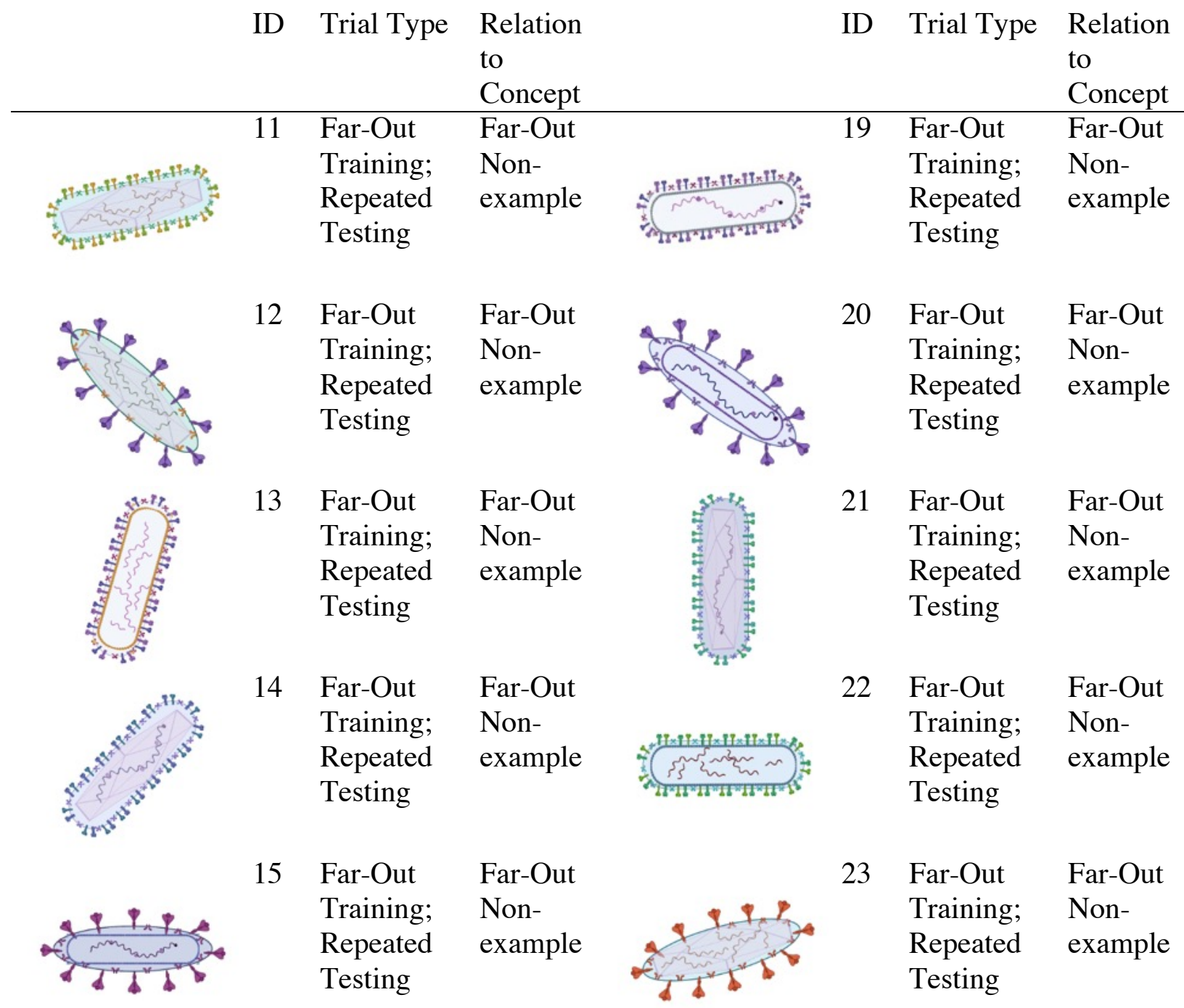

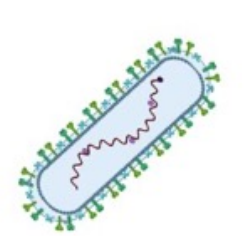

16 Far-Out Far-Out

Training; Non-

Repeated example

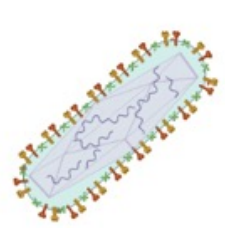

24 Far-Out Far-Out

Training; Non-

Testing

Repeated example

Testing

17 Far-Out Far-Out

Training; Non-

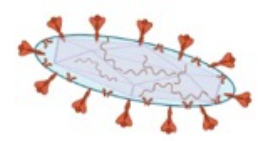

Repeated example

Testing

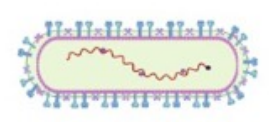

25

Untrained Example

Testing

(Far-Out)

18 Far-Out Far-Out

Training; Non-

Repeated example

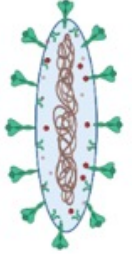

26

Untrained Example

Testing

(Far-Out) 


\begin{tabular}{|c|c|c|c|c|c|c|}
\hline & ID & Trial Type & $\begin{array}{l}\text { Relation } \\
\text { to } \\
\text { Concept }\end{array}$ & ID & Trial Type & $\begin{array}{l}\text { Relation } \\
\text { to } \\
\text { Concept }\end{array}$ \\
\hline & 27 & $\begin{array}{l}\text { Untrained } \\
\text { Testing } \\
\text { (Far-Out) }\end{array}$ & Example & 35 & $\begin{array}{l}\text { Untrained } \\
\text { Testing } \\
\text { (Close-In) }\end{array}$ & Example \\
\hline 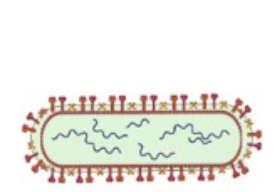 & 28 & $\begin{array}{l}\text { Untrained } \\
\text { Testing } \\
\text { (Far-Out) }\end{array}$ & Example & 36 & $\begin{array}{l}\text { Untrained } \\
\text { Testing } \\
\text { (Close-In) }\end{array}$ & Example \\
\hline & 29 & $\begin{array}{l}\text { Untrained } \\
\text { Testing } \\
\text { (Far-Out) }\end{array}$ & Example & 37 & $\begin{array}{l}\text { Untrained } \\
\text { Testing } \\
\text { (Far-Out) }\end{array}$ & $\begin{array}{l}\text { Far-Out } \\
\text { Non- } \\
\text { example }\end{array}$ \\
\hline & 30 & $\begin{array}{l}\text { Untrained } \\
\text { Testing } \\
\text { (Far-Out) }\end{array}$ & Example & 38 & $\begin{array}{l}\text { Untrained } \\
\text { Testing } \\
\text { (Far-Out) }\end{array}$ & $\begin{array}{l}\text { Far-Out } \\
\text { Non- } \\
\text { example }\end{array}$ \\
\hline & 31 & $\begin{array}{l}\text { Untrained } \\
\text { Testing } \\
\text { (Close-In) }\end{array}$ & Example & 39 & $\begin{array}{l}\text { Untrained } \\
\text { Testing } \\
\text { (Far-Out) }\end{array}$ & $\begin{array}{l}\text { Far-Out } \\
\text { Non- } \\
\text { example }\end{array}$ \\
\hline & 32 & $\begin{array}{l}\text { Untrained } \\
\text { Testing } \\
\text { (Close-In) }\end{array}$ & Example & 40 & $\begin{array}{l}\text { Untrained } \\
\text { Testing } \\
\text { (Far-Out) }\end{array}$ & $\begin{array}{l}\text { Far-Out } \\
\text { Non- } \\
\text { example }\end{array}$ \\
\hline & 33 & $\begin{array}{l}\text { Untrained } \\
\text { Testing } \\
\text { (Close-In) }\end{array}$ & Example & 41 & $\begin{array}{l}\text { Untrained } \\
\text { Testing } \\
\text { (Far-Out) }\end{array}$ & $\begin{array}{l}\text { Far-Out } \\
\text { Non- } \\
\text { example }\end{array}$ \\
\hline & 34 & $\begin{array}{l}\text { Untrained } \\
\text { Testing } \\
\text { (Close-In) }\end{array}$ & Example & 42 & $\begin{array}{l}\text { Untrained } \\
\text { Testing } \\
\text { (Far-Out) }\end{array}$ & $\begin{array}{l}\text { Far-Out } \\
\text { Non- } \\
\text { example }\end{array}$ \\
\hline
\end{tabular}




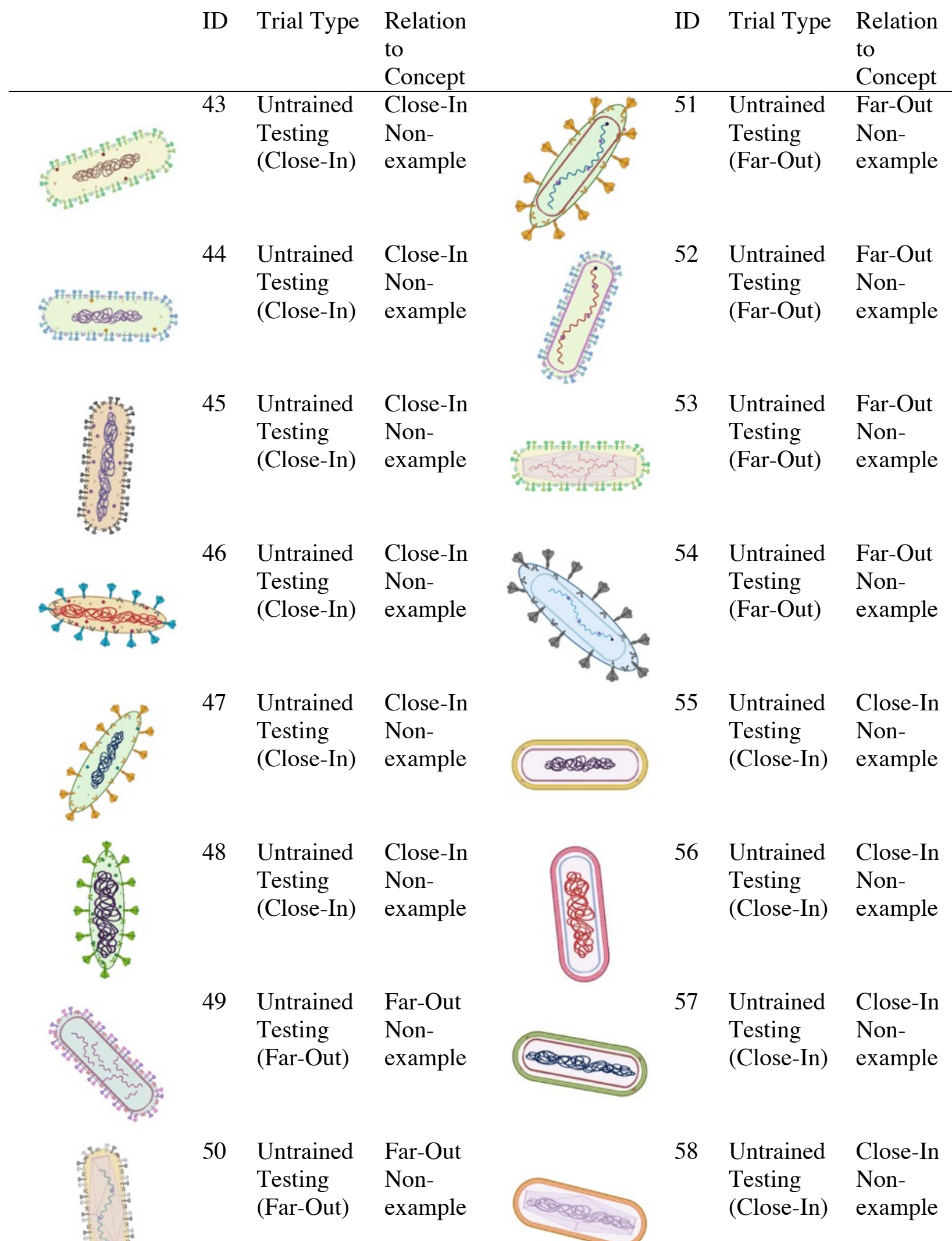




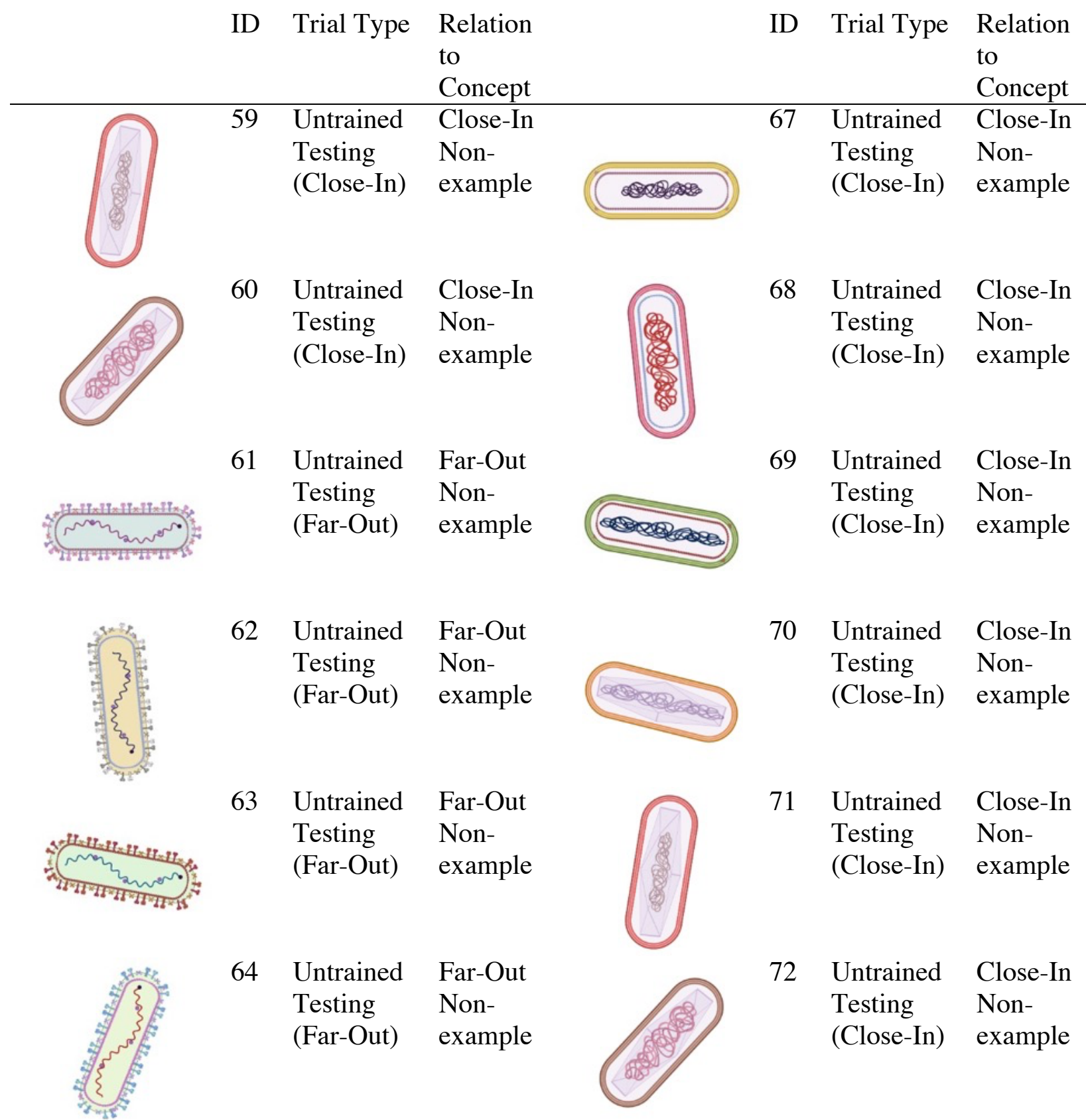

65 Untrained Far-Out Testing Non(Far-Out) example

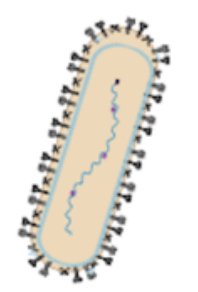

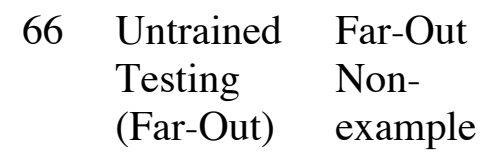




\section{Plant Cells}

\begin{tabular}{|c|c|c|c|c|c|c|}
\hline & ID & Trial Type & $\begin{array}{l}\text { Relation } \\
\text { to } \\
\text { Concept }\end{array}$ & ID & Trial Type & $\begin{array}{l}\text { Relation } \\
\text { to } \\
\text { Concept }\end{array}$ \\
\hline$\%$ & 1 & $\begin{array}{l}\text { All } \\
\text { Training; } \\
\text { Repeated } \\
\text { Testing }\end{array}$ & Example & 8 & $\begin{array}{l}\text { Examples } \\
\text { Only } \\
\text { Training; } \\
\text { Repeated } \\
\text { Testing }\end{array}$ & Example \\
\hline $\begin{array}{l}0 \\
0 \\
0\end{array}$ & 2 & $\begin{array}{l}\text { All } \\
\text { Training; } \\
\text { Repeated } \\
\text { Testing }\end{array}$ & Example & 9 & $\begin{array}{l}\text { Examples } \\
\text { Only } \\
\text { Training; } \\
\text { Repeated } \\
\text { Testing }\end{array}$ & Example \\
\hline & 3 & $\begin{array}{l}\text { All } \\
\text { Training; } \\
\text { Repeated } \\
\text { Testing }\end{array}$ & Example & 10 & $\begin{array}{l}\text { Examples } \\
\text { Only } \\
\text { Training; } \\
\text { Repeated } \\
\text { Testing }\end{array}$ & Example \\
\hline & 4 & $\begin{array}{l}\text { All } \\
\text { Training; } \\
\text { Repeated } \\
\text { Testing }\end{array}$ & Example & 11 & $\begin{array}{l}\text { Examples } \\
\text { Only } \\
\text { Training; } \\
\text { Repeated } \\
\text { Testing }\end{array}$ & Example \\
\hline & 5 & $\begin{array}{l}\text { All } \\
\text { Training; } \\
\text { Repeated } \\
\text { Testing }\end{array}$ & Example & 12 & $\begin{array}{l}\text { Examples } \\
\text { Only } \\
\text { Training; } \\
\text { Repeated } \\
\text { Testing }\end{array}$ & Example \\
\hline & 6 & $\begin{array}{l}\text { All } \\
\text { Training; } \\
\text { Repeated } \\
\text { Testing }\end{array}$ & Example & 13 & $\begin{array}{l}\text { Examples } \\
\text { Only } \\
\text { Training; } \\
\text { Repeated } \\
\text { Testing }\end{array}$ & Example \\
\hline$(9-1)$ & 7 & $\begin{array}{l}\text { Examples } \\
\text { Only } \\
\text { Training; } \\
\text { Repeated } \\
\text { Testing }\end{array}$ & Example & 14 & $\begin{array}{l}\text { Examples } \\
\text { Only } \\
\text { Training; } \\
\text { Repeated } \\
\text { Testing }\end{array}$ & Example \\
\hline
\end{tabular}




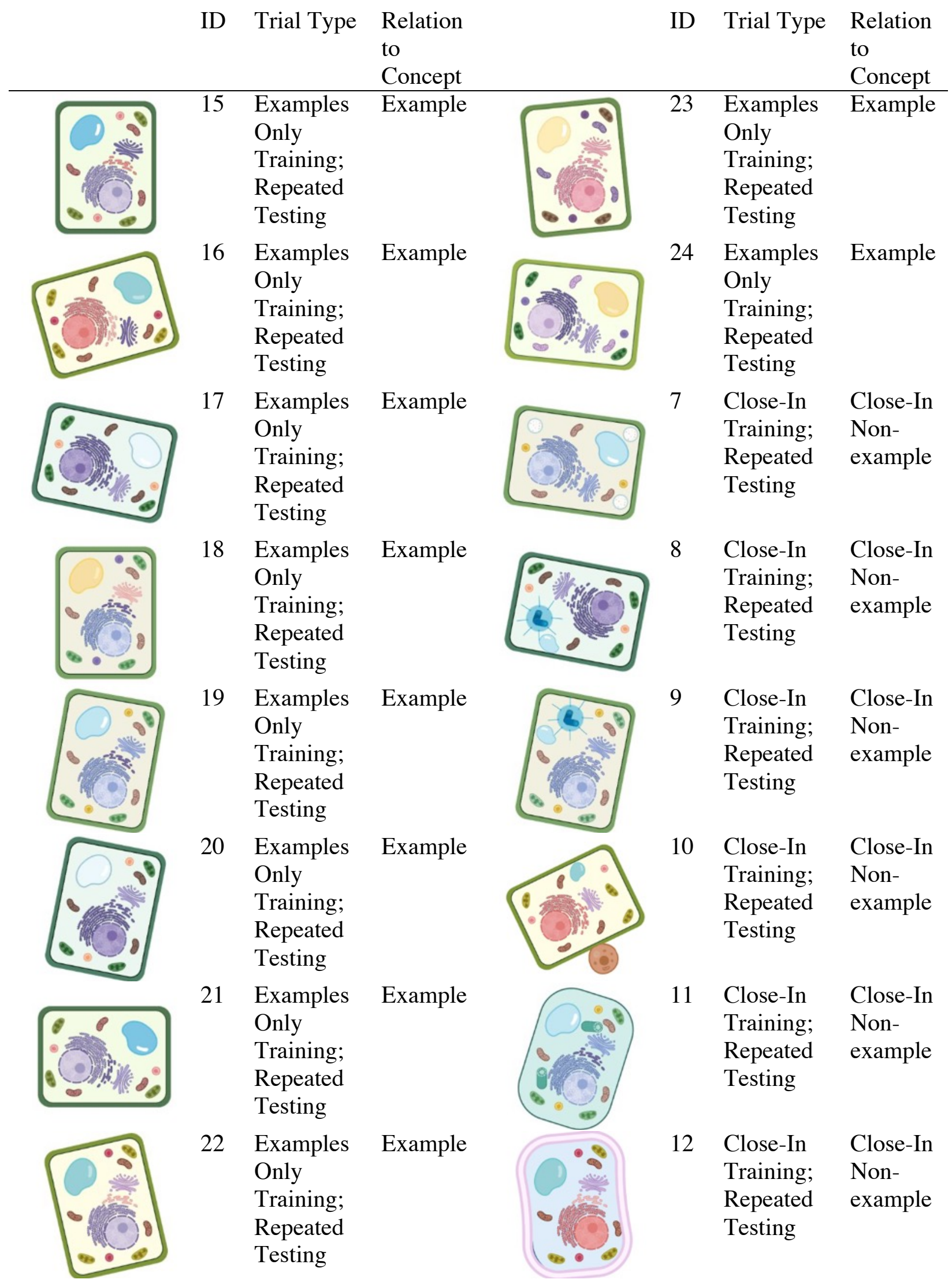




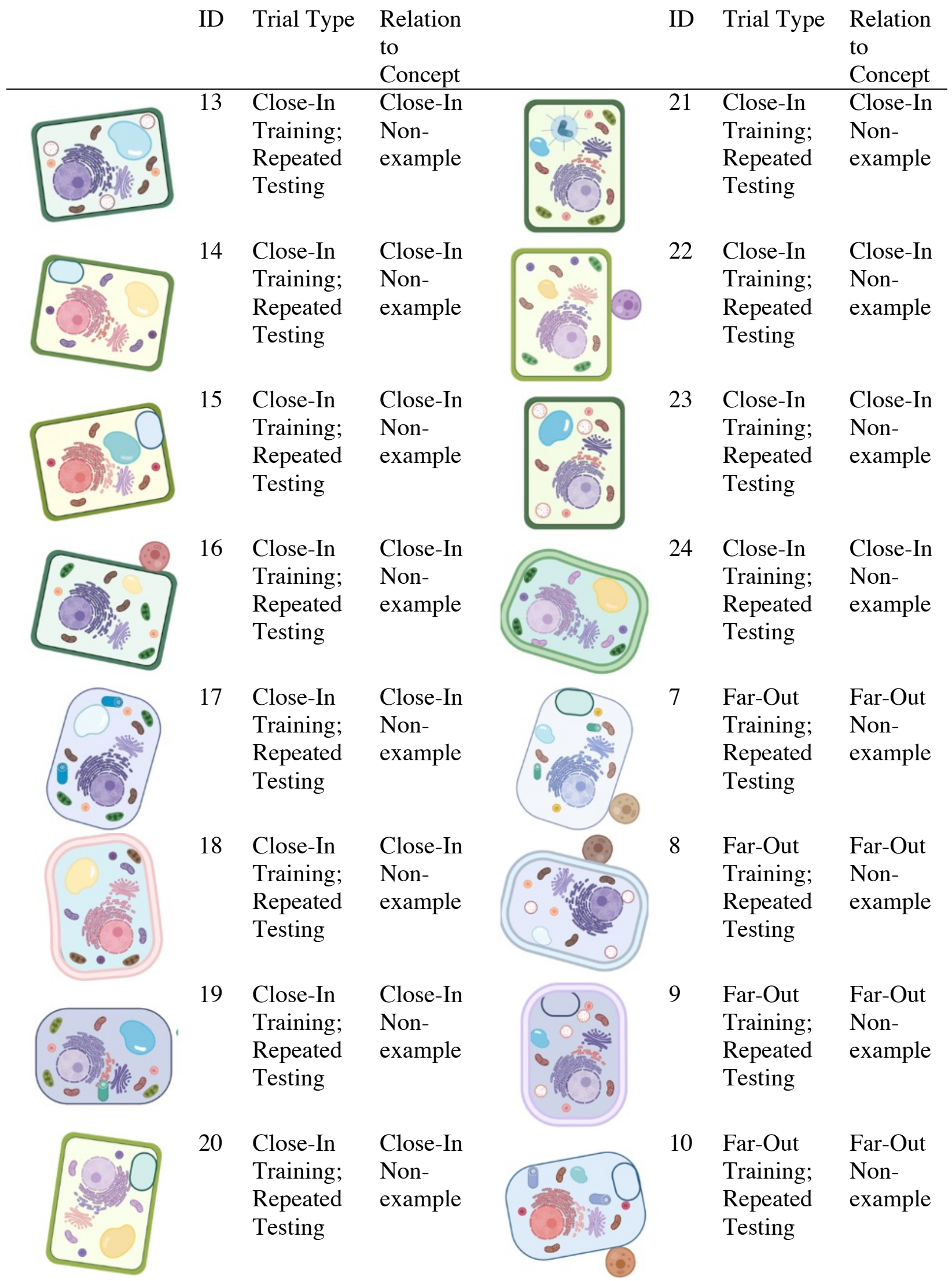




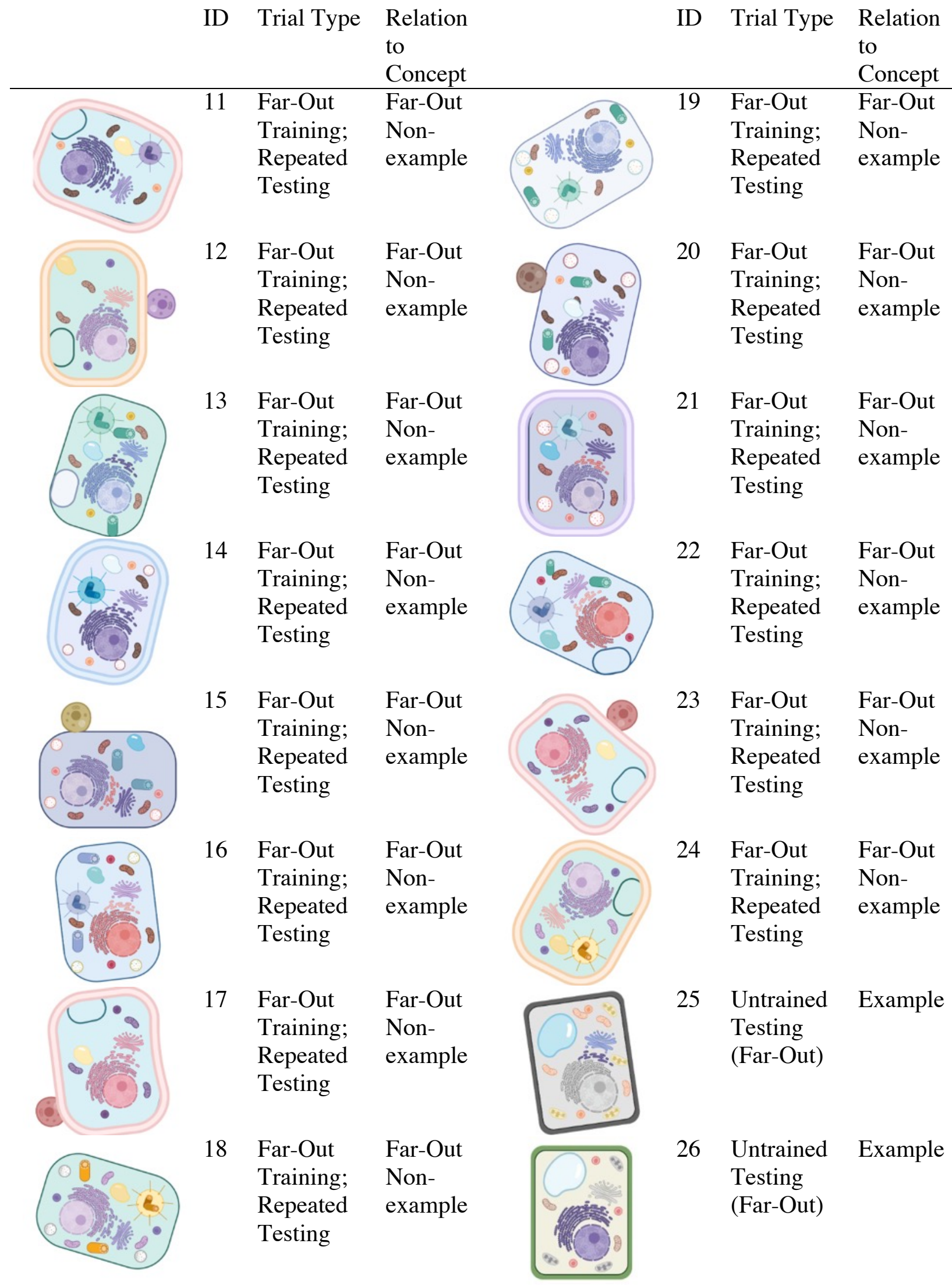




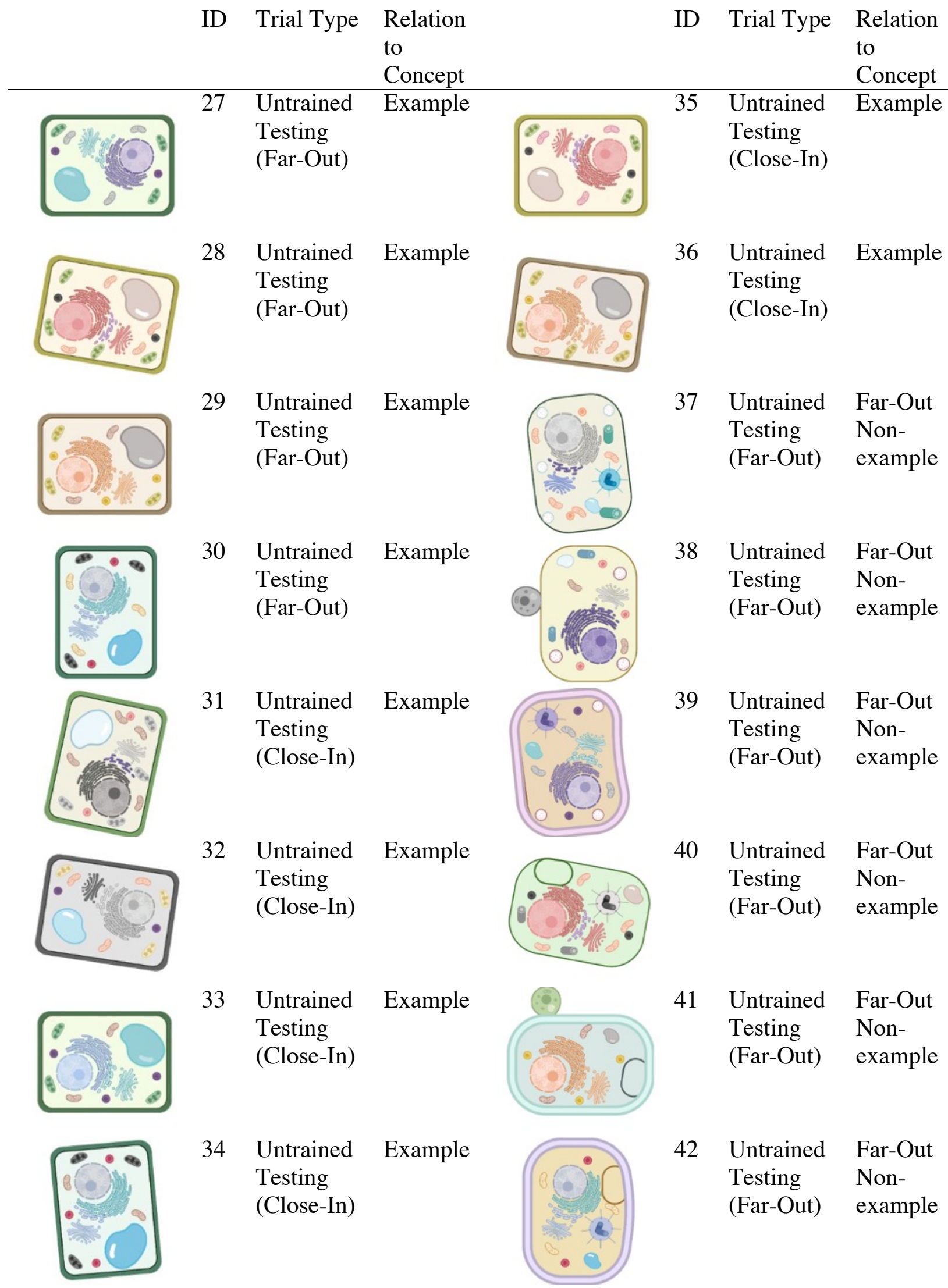




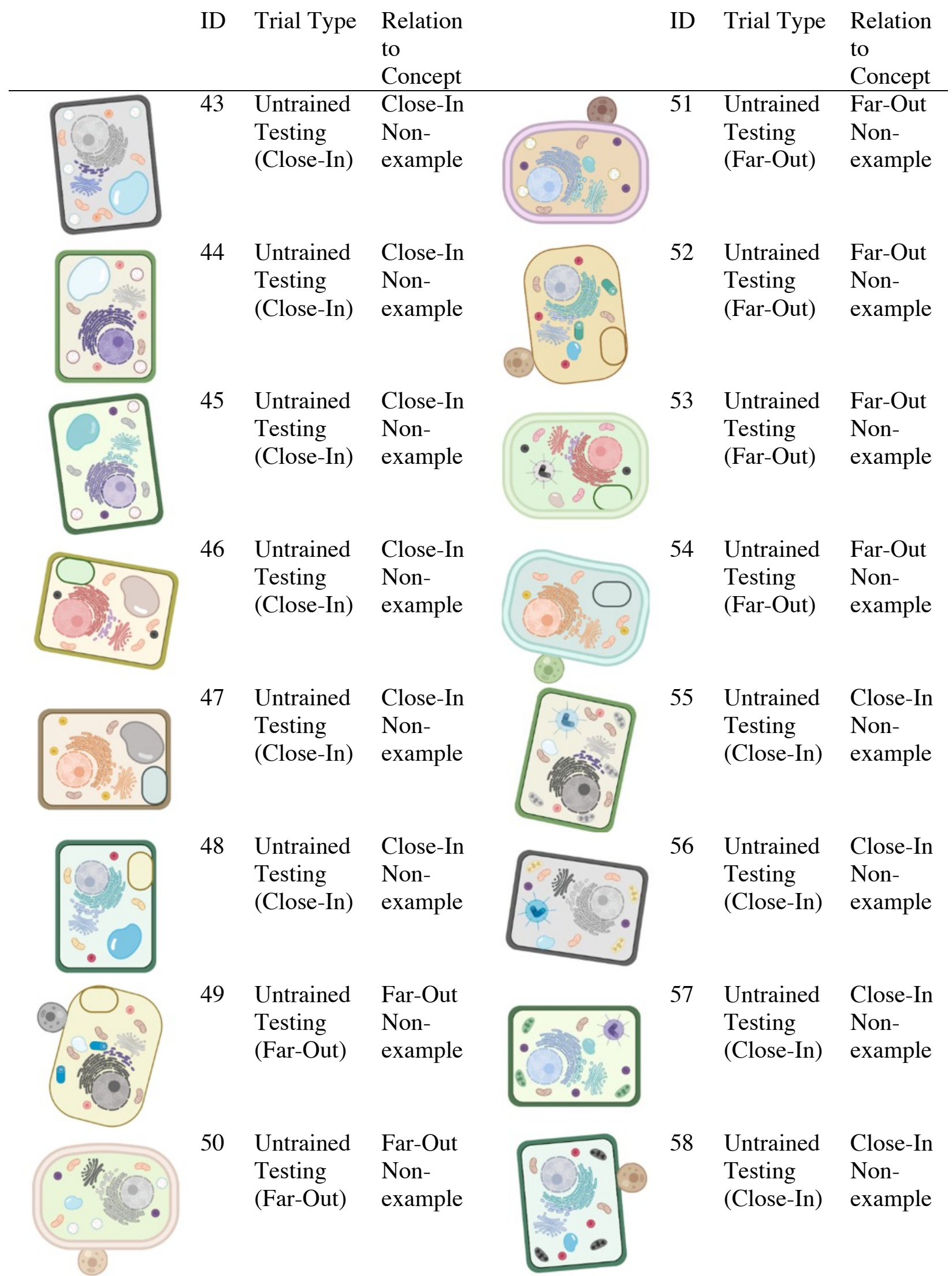




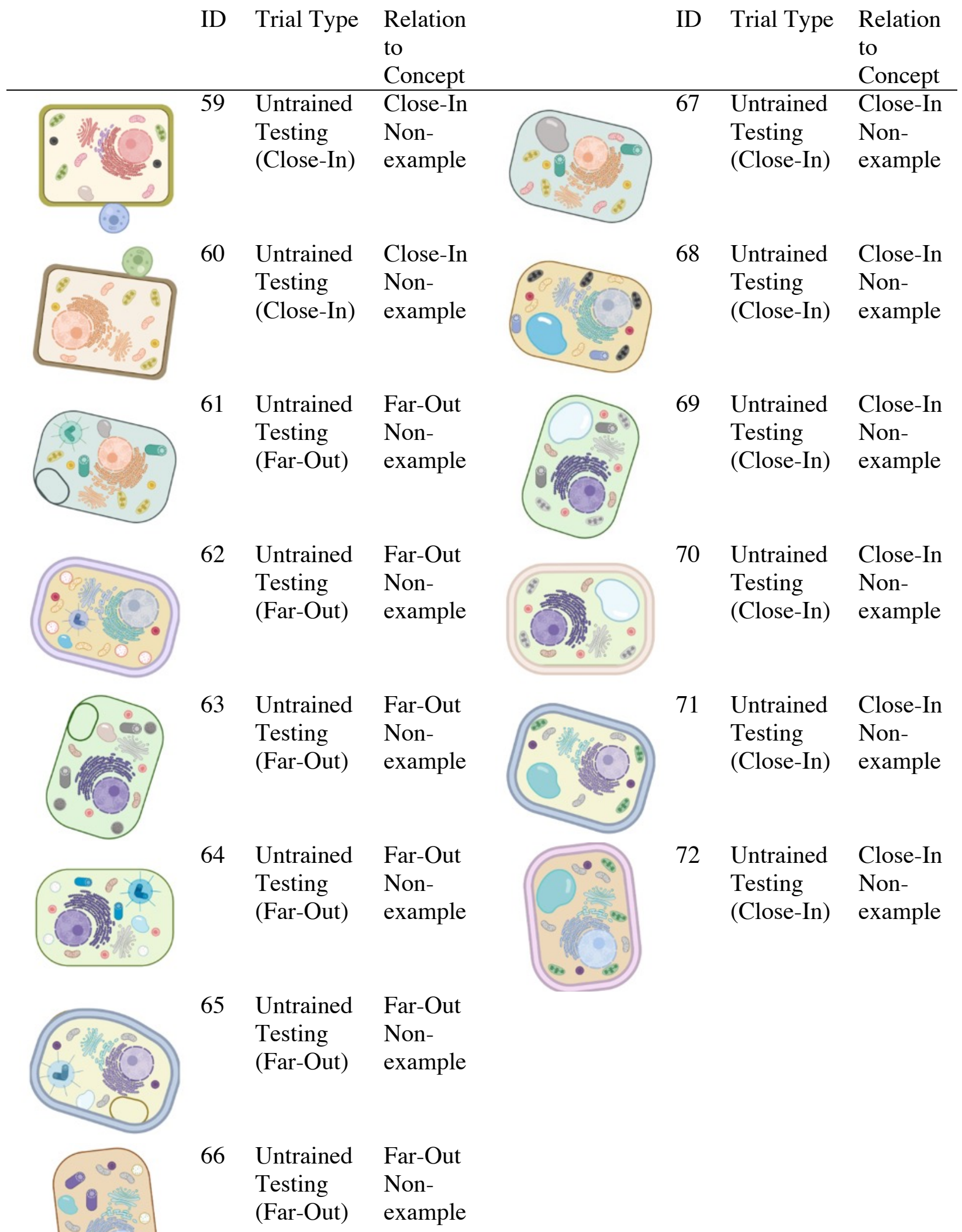




\section{Appendix K: Experiment 2 Baseline/Test Trial Examples}

As in Experiment 1, test trials were divided into three stimulus arrangements: trials with training stimuli (shown in Appendix F), trials with untrained examples and close-in nonexamples (shown on this page), and trials with untrained examples and far-out nonexamples (shown on the following page). Regardless of the training condition, trials with untrained stimuli were the same for each set of stimuli.

\section{Trials with Untrained Close-In Nonexamples}

\section{Sensory Neurons:}

Stimulus II contains all three relevant features, making it an example of a sensory neuron. Selecting Stimulus II would be the only correct response. Stimuli I, III, and IV each lack one feature, making them close-in nonexamples of sensory neurons. Stimulus I lacks dendrites on only the end of the neuron, Stimulus III lacks sensory receptors, and Stimulus IV lacks a cell body on a side branch.

\section{Bacteria:}

Stimulus IV contains all three relevant features, making it an example of bacteria. Selecting Stimulus IV would be the only correct response. Stimuli I, II, and III each lack one feature, making them close-in nonexamples of bacteria cells. Stimulus I lacks ribosomes, Stimulus II lacks HA/NA surface proteins, and Stimulus III lacks DNA.

\section{Plant Cells:}

Stimulus I contains all three relevant features, making it an example of a plant cell. Selecting stimulus I would be the only correct response. Stimuli II, III, and IV each lack one feature, making them close-in nonexamples of plant cells. Stimulus II lacks chloroplasts, and Stimulus III lacks a large vacuole, and Stimulus IV lacks a cell wall.

*Roman numerals did not appear on screen and are for reference only.
I

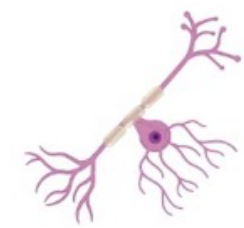

III

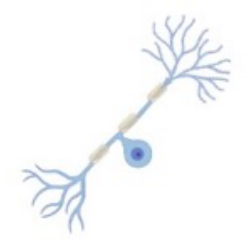

I

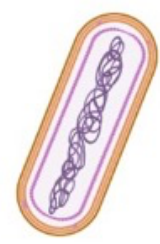

III

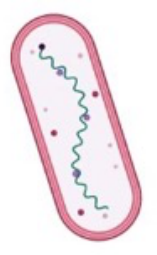

I

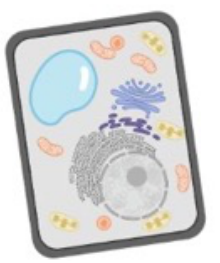

III

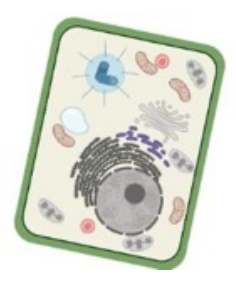

II

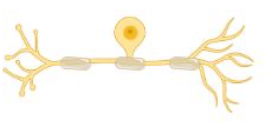

IV

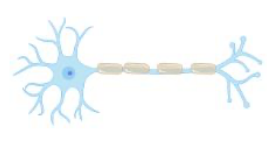

II

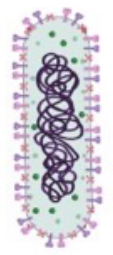

IV

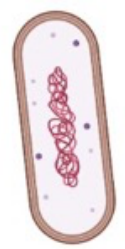

II

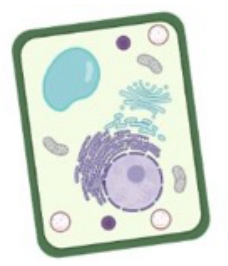

IV

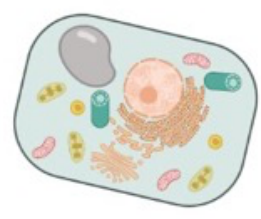




\section{Trials with Untrained Far-Out Nonexamples}

\section{Sensory Neuron:}

Stimulus I contains all three relevant features, making it an example of a sensory neuron. Selecting Stimulus I would be the only correct response. Stimuli II, III, and IV lack all three relevant features, making them far-out nonexamples of sensory neurons.

\section{Bacteria:}

Stimulus II contains all three relevant features, making it an example of a bacteria cell. Selecting Stimulus II would be the only correct response. Stimuli I, III, and IV lack all three relevant features, making them far-out nonexamples of bacteria.

\section{Plant Cells:}

Stimulus III contains all three relevant features, making it an example of a plant cell. Selecting Stimulus III would be the only correct response. Stimuli I, II, and IV lack all three relevant features, making them far-out nonexamples of plant cells.

I

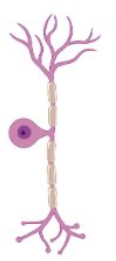

III

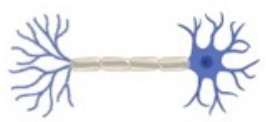

IV

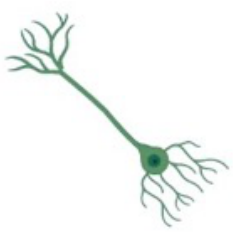

I

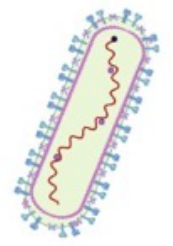

II

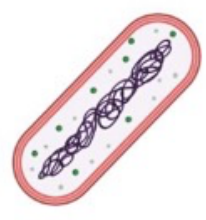

III

IV
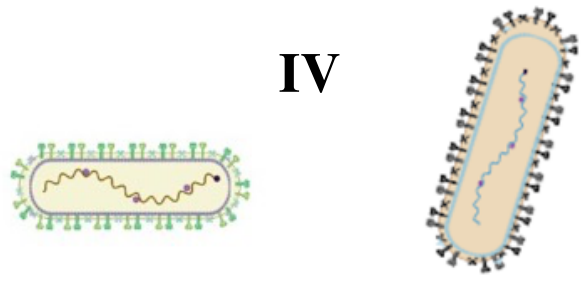

I

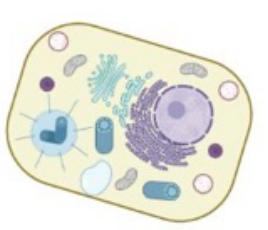

III

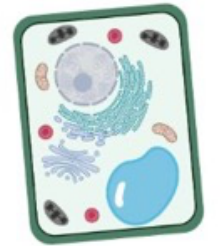

II

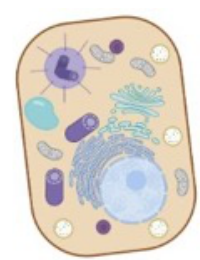

IV

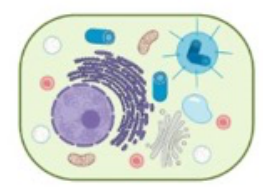
did not appear on screen and are for reference only. 


\section{Appendix L: Pre-Experimental Stimuli Evaluations (Experiment 2)}

After the stimuli were created, three adults affiliated with the author completed the fulllength pilot-test of the experiment. Baseline response accuracy was low for two participants for all three stimulus sets. For one participant, baseline responding was highly accurate for trials with far-out nonexamples in one condition, though not high enough for the participant to be dismissed. The results did not support the need for additional alterations to the stimuli. However, additional changes to the stimuli were made in response to responding during Experiment 2 (see Experimental Modifications). 


\section{Appendix M: Experiment 2 Training Trials Examples}

Note that there are three sets of stimuli for each concept; one set for each training condition. A different concept was taught in each training condition, but the concept assigned to each training condition were counterbalanced across participants.

Sensory Neurons

The three relevant features for an example of sensory neurons are: cell body on side branch, sensory receptors, and dendrites on only end of neuron

\section{If used for the Examples Only condition:}

Although these stimuli vary in many ways, all four stimuli contain the three critical features. Selecting any of the four stimuli would be a correct response.

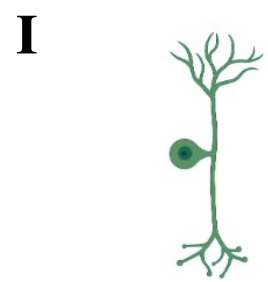

III

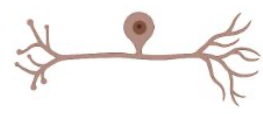

II

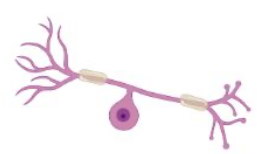

IV

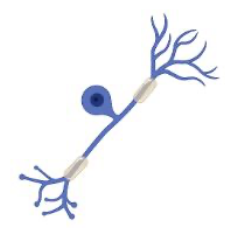

If used for the Close-In Nonexamples condition:

Stimulus III contains all three relevant features, making it an example of sensory neurons. Selecting Stimulus III would be the only correct response. Stimuli I, II, and IV each lack one feature, making them close-in nonexamples of sensory neurons. Stimulus II lacks a cell body on a side branch, Stimulus III lacks segmented RNA strands, and Stimulus IV lacks sensory receptors.

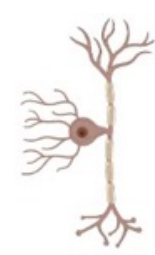

III

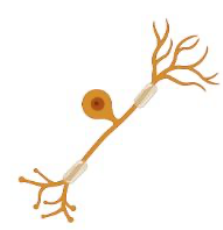

II

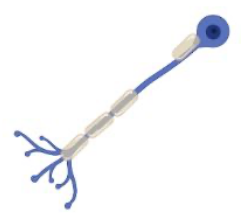

IV

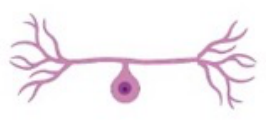

\section{If used for the Far-Out Nonexamples condition:}

Stimulus I contains all three relevant features, making it an example of sensory neurons. Selecting Stimulus I would be the only correct response. Stimuli II, III, and IV lack all three relevant features, making them far-out nonexamples of influenza.
*Roman numerals did not appear on screen and are for reference only.
I

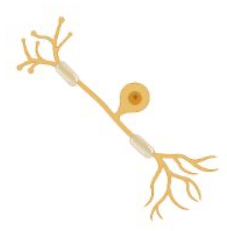

II

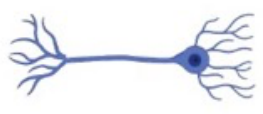

IV

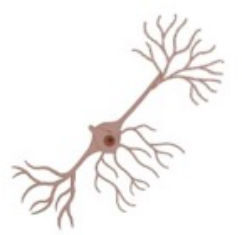


Bacteria Cells

The three relevant features for an example of bacteria are: surface proteins, DNA, and ribosomes.

\section{If used for the Examples Only condition:}

Although these stimuli vary in many ways, all four stimuli contain the three critical features. Selecting any of the four stimuli would be a correct response.
I

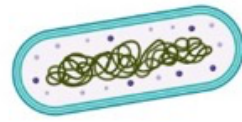

III

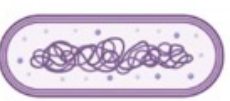

II

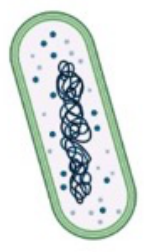

IV

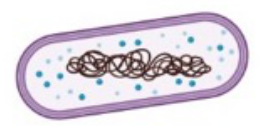

If used for the Close-In Nonexamples condition:

Stimulus III contains all three relevant features, making it an example of bacteria. Selecting Stimulus III would be the only correct response. Stimuli I, II, and IV each lack one feature, making them close-in nonexamples of bacteria cells. Stimuli I and II lack HA/NA surface proteins, and Stimulus IV lacks DNA.
I

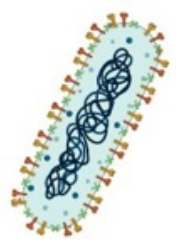

III

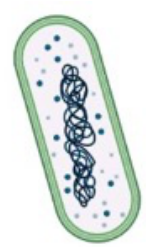

II

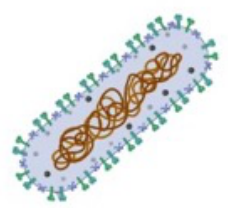

IV

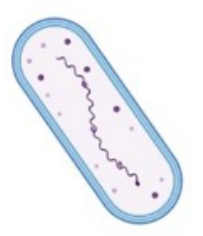

If used for the Far-Out Nonexamples condition:

Stimulus IV contains all three relevant features, making it an example of bacteria. Selecting Stimulus IV would be the only correct response. Stimuli I, II, and III lack all three relevant features, making them far-out nonexamples of influenza.
I

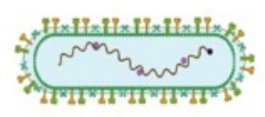

II

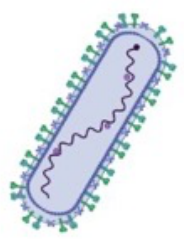

IV
*Roman numerals did not appear on screen and are for reference only.
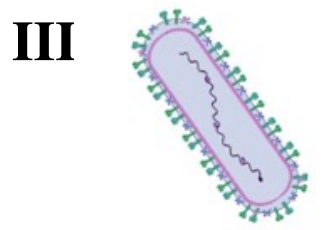


\section{Plant Cells}

The three relevant features for an example of plant cells are: large vacuole, cell wall, and chloroplasts.

\section{If used for the Examples Only condition:}

Although these stimuli vary in many ways, all four stimuli contain the three critical features. Selecting any of the four stimuli would be a correct response.
I

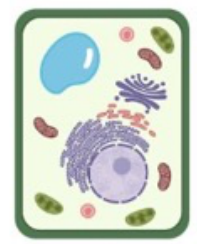

III

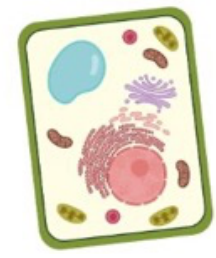

II

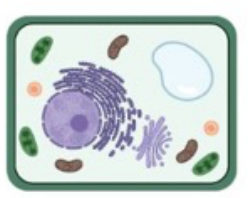

IV

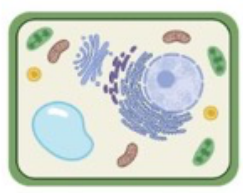

If used for the Close-In Nonexamples condition:

Stimulus IV contains all three relevant features, an example of a plant cell. Selecting Stimulus IV the only correct response. Stimuli I, II, and III each feature, making them close-in nonexamples of plant Stimulus I lacks a cell wall, Stimulus II lacks chloroplasts, and Stimulus III lacks a large vacuole.
I

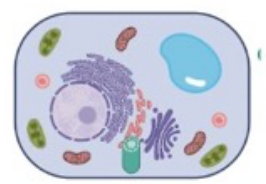

III

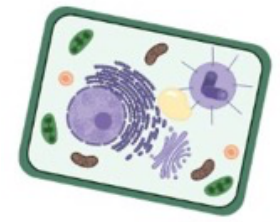

II

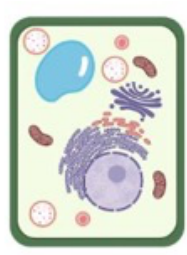

IV

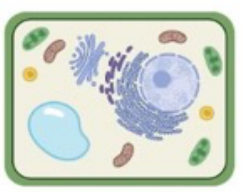

If used for the Far-Out Nonexamples condition:

Stimulus I contains all three relevant features, making it an example of a plant cell. Selecting Stimulus I would be the only correct response. Stimuli II, III, and IV lack all three relevant features, making them far-out nonexamples of plant cells.
I

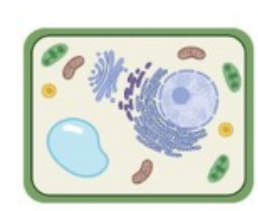

III

*Roman numerals did not appear on screen and are for reference only.
II

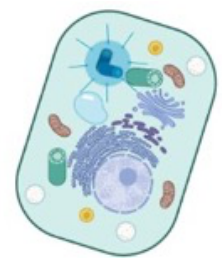

IV
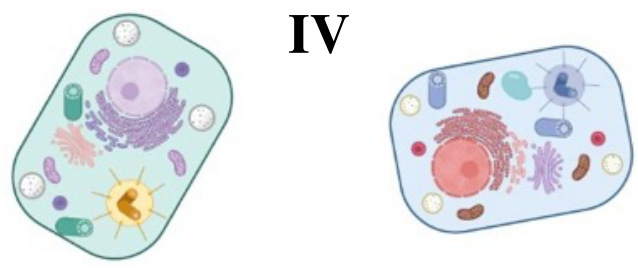


\section{Appendix N: Description of Concept and Strategies of Selection}

What do you think the name of the concept you were taught was?

How did you decide which image to select? 


\section{Appendix O: Experiment 2 Incomplete Participants}
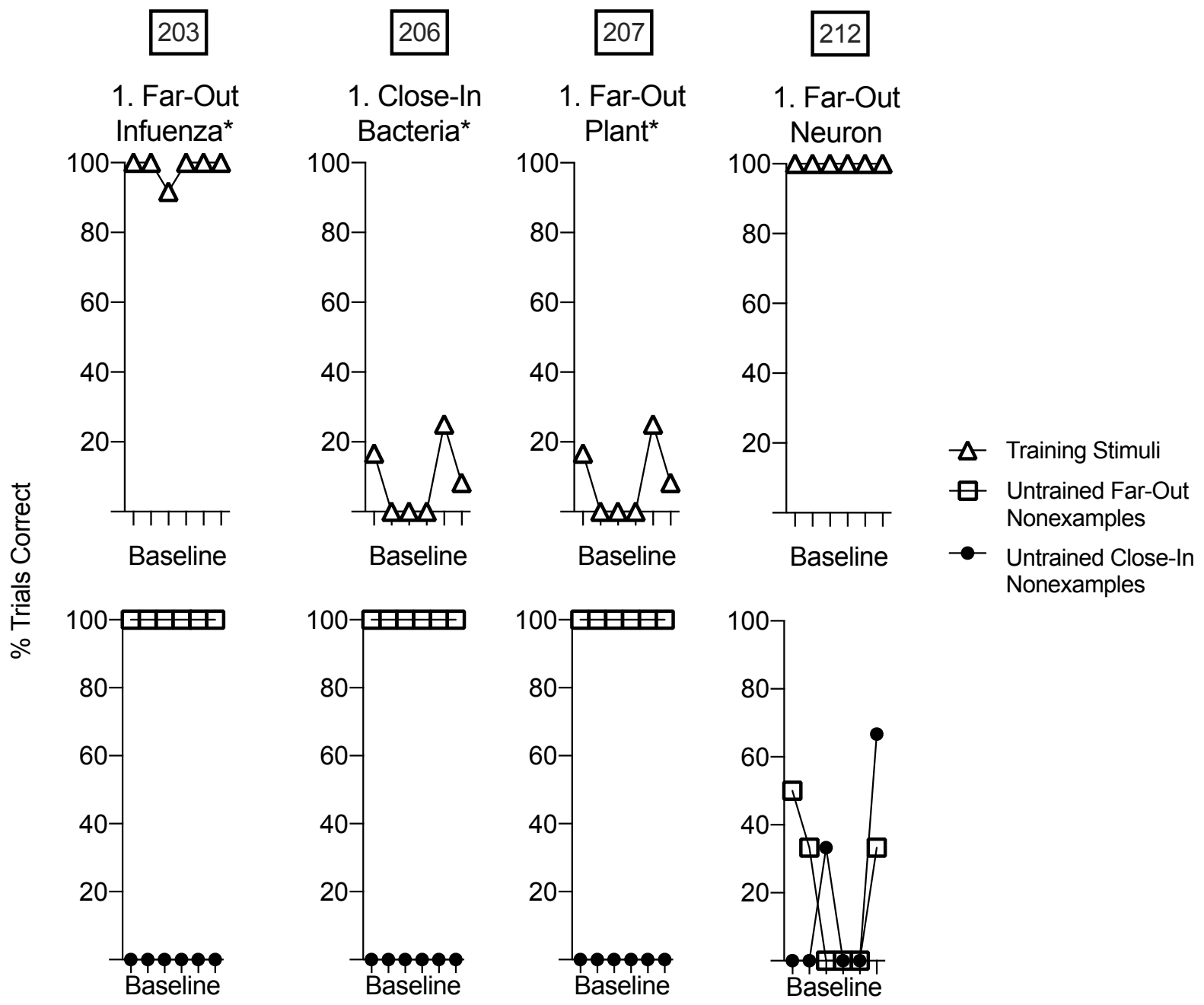

Blocks

* Original set of experimental stimuli was used. Each relevant feature was replaced with only one replacement feature.

Note. Training blocks are indicated by the solid, vertical line between baseline and testing blocks, though data from these blocks are not displayed. The conditions are indicated by the names. The number before the condition name indicates the order in which that condition was experienced. 


\section{Appendix P: Survey Responses Following Training in Each Condition}

\begin{tabular}{|c|c|c|}
\hline ID \# & Name & Description \\
\hline 202 & Differentiation & $\begin{array}{l}\text { Examples Only } \\
\text { In order to choose an image, I looked at each and examined the } \\
\text { direction, border type, and colors, and then I determined which } \\
\text { image was different than the rest and chose that one. }\end{array}$ \\
\hline 204 & Comparison & I picked whichever was the most different from the other ones. \\
\hline 205 & $\begin{array}{l}\text { Which one is } \\
\text { different? }\end{array}$ & $\begin{array}{l}\text { Based off of how many nodules there were in the middle of each } \\
\text { stick thing, if there was an object in the middle of the stick thing, } \\
\text { if that object had squiggles coming out of it, which way all the } \\
\text { sticks were facing, the color of the sticks. }\end{array}$ \\
\hline 208 & Not sure & Color, orientation, shapes \\
\hline $\begin{array}{l}208 \\
\text { (Old }\end{array}$ & Cells/Biology & Color, rotation, specific shapes, differences between images \\
\hline \multicolumn{3}{|l|}{ Stimuli) } \\
\hline 211 & $\begin{array}{l}\text { Not sure on } \\
\text { this one, it } \\
\text { seemed like } \\
\text { no matter } \\
\text { which I picked } \\
\text { it was the } \\
\text { right answer }\end{array}$ & Just picked at random \\
\hline
\end{tabular}

\section{Far-Out Nonexamples}

202 Differentiation I chose images based off of which one looked the most different from the group. If one didn't seem to fit in, I would pick it.

202 Differentiation I decided what images to click on by determining which one of the

(Old

Stimuli)

205 Which one doesn't belong

208

209

211 Uniqueness images did not match the others 3 images. I clicked on the image that was distinct and different from the others.

If three of the four images had spikes on the outside I picked the one that didn't have spikes. If three of the four images didn't have spikes I picked the one that did.

Which object was different then the other three like if three had spikes and one did not 


\section{Close-In Nonexamples}

201 Something I based it off of what the previous technique was for the correct about risky image.

and safety

202 Memorization I always picked the image with the large squiggle and the polka dot background.

204 Memorization I looked at the different characteristics of the molecules and I clicked on the one that was similar to the one I got right beforehand

205 Which one whichever image was the most basic and didn't have anything that doesn't belong made it special.

209 Operant I learned to recognize patterns of the different images and what conditioning differentiated them. When told it was the right answer I would keep a characteristic of that image in mind based on how distinct it was compared to the others. When not told what was correct, I tried to remember the characteristics and individual details that the right image has from the previous and identify it out of the options given.

210 Pattern The line with a protruding circular node attached the middle, with identification one end made of small rounded branches and the other of medium elongated branches appeared in every selection. The colors of the shapes and clear bars on the center line had no meaning that I could discern.

211 Memorization After the second session it came to me that I had memorize certain images and then that was when I figured it out. 


\section{Appendix Q: Description from Participants Who Responded Correctly During Training}

201: "Today I was using the strategy that I learned when I was told what was correct or incorrect the last time. Basically, I went by process of elimination to see where there was consistency and appeared each time. Once I realized which one was being showed each time then I was able to just keep clicked that one."

204: "The strategy I used was first I picked a random picture that I liked and tried to remember the distinct features that were in it. When it went to the next slide I noticed that there was only one that looked like it and none of the other photos looked like ones from the previous slide, so I assumed that was the correct answer. Once I realized this I kept clicking the ones that had all the similar features I saw at the beginning."

208: "At first, I was going off of color, shapes, and orientation, especially with the cells since I knew a little bit of how they normally should look like. Then what I did was start with the pictures I like the most, until I see a trend in pictures over multiple questions. Specifically, with the sections that showed if I was wrong or not, I tested the bounds of what would be wrong and then answered using that information. At times I doubted I got so many right so every now and then I intentionally tried to be wrong to see if it was going to be right no matter what, or not."

210: "As for the strategy I used, it was mainly based off of the pattern I found for the first trial. I gave each trial nicknames to better describe them. The first trial, the nerves, I struggled with until I reached the portion where it told me what was correct. I was overthinking it by a lot. The "branches" at the end of the nerve length had different sizes and shapes, rounded and sharp. The "node", the circular piece found on different parts of the nerve was always in the same place, in the middle. So the "correct" answer was finding the nerve shape with one small sized rounded branch, one small sharp branch, and a node hanging off the center with no additional branches coming out of it. This was the correct answer regardless of the bars, color, or shape orientation.

The second trial, the cells, I used the same thinking. In order to find the "correct" one, I guessed through a few rounds and tried to find specific characteristics that kept repeating. Regardless of color or orientation, I found common pieces occurring in at least one cell. After each round, I was able to solidify what the "correct" characteristics were.

The final trial, cells (pt. 2), I used the same strategy. Find reoccurring shapes and characteristics, and then stick with that. This ones, I was the most unsure of. I found the cell with the same characteristics but didn't always reoccur in the same way. So I tried using the rounds where there was one normal cell and three extremely different ones. I studied what the normal cells looked like and tried to find ones in other rounds as similar as possible." 


\section{Appendix R: Summary of Outcomes across Both Experiments}

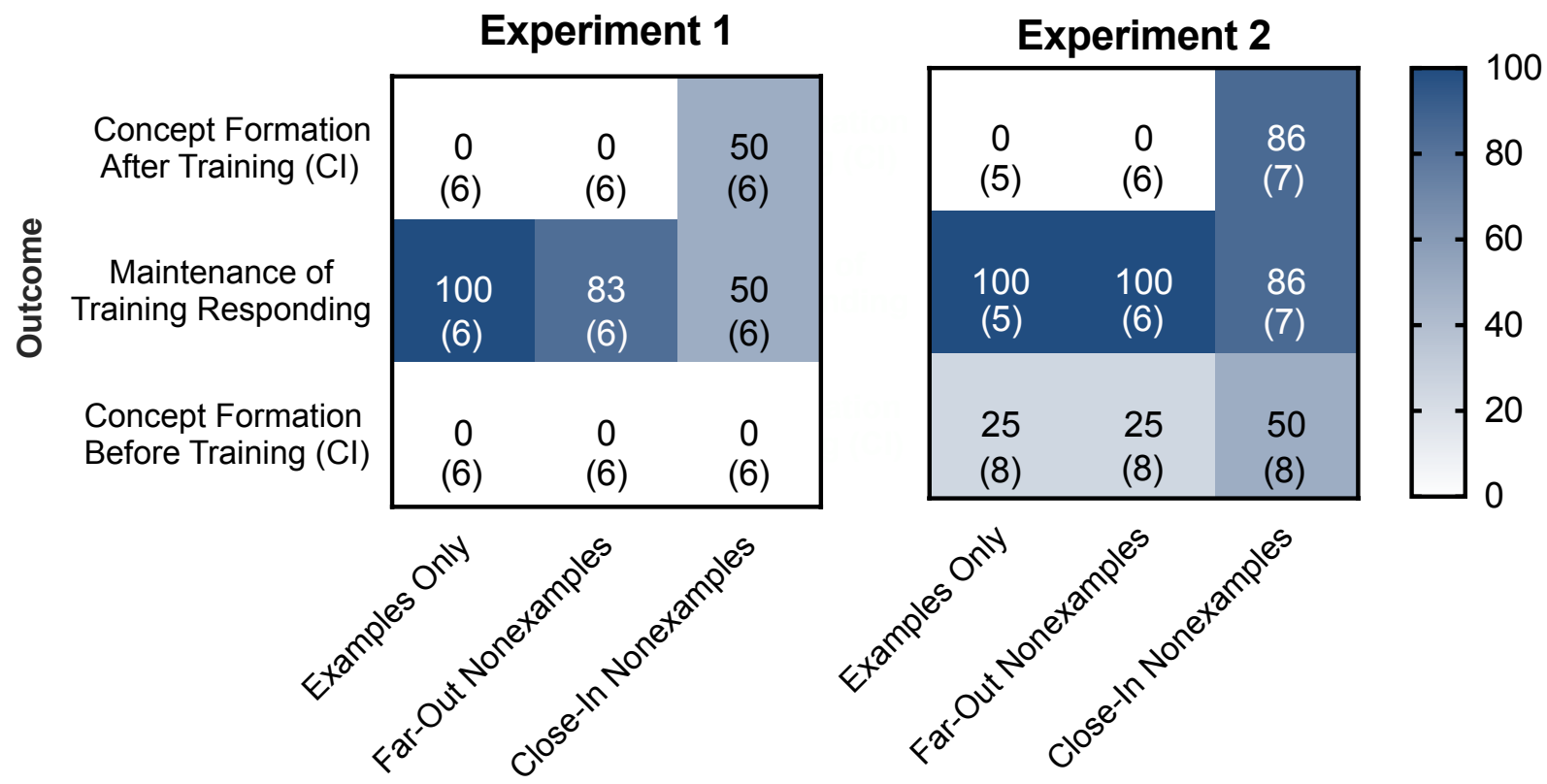

Condition

Note. Each square contains the percentage of participants that met the outcome (listed on the left) in each condition (listed on the bottom). A participant met the outcome "Concept Formation After Training" if they responded correctly on all trials with untrained close-in nonexamples during at least three testing blocks. A participant met the outcome "Maintenance of Training Responding" if they responded correctly on at least 11 of 12 trials with training stimuli during at least three testing blocks. A participant met the outcome "Concept Formation Before Training" if they responded correctly on all trials with untrained close-in nonexamples during at least three baseline blocks. The number of participants that met the requirements to be included in the calculation is shown in parentheses. 